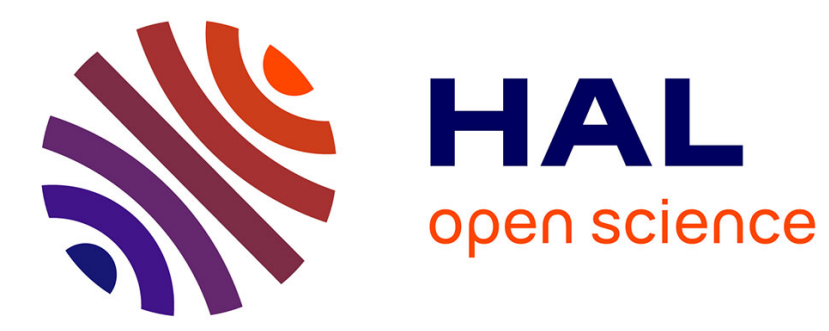

\title{
Nonlinear Robust Periodic Output Regulation of Minimum Phase Systems
}

\author{
Daniele Astolfi, Laurent Praly, Lorenzo Marconi
}

\section{To cite this version:}

Daniele Astolfi, Laurent Praly, Lorenzo Marconi. Nonlinear Robust Periodic Output Regulation of Minimum Phase Systems. Mathematics of Control, Signals, and Systems, inPress. hal-03335543

\section{HAL Id: hal-03335543 \\ https://hal.science/hal-03335543}

Submitted on 13 Sep 2021

HAL is a multi-disciplinary open access archive for the deposit and dissemination of scientific research documents, whether they are published or not. The documents may come from teaching and research institutions in France or abroad, or from public or private research centers.
L'archive ouverte pluridisciplinaire HAL, est destinée au dépôt et à la diffusion de documents scientifiques de niveau recherche, publiés ou non, émanant des établissements d'enseignement et de recherche français ou étrangers, des laboratoires publics ou privés. 


\title{
Nonlinear Robust Periodic Output Regulation of Minimum Phase Systems
}

\author{
Daniele Astolfi · Laurent Praly · Lorenzo \\ Marconi
}

Received: September 13, 2021/ Accepted: date

\begin{abstract}
In linear systems theory it's a well known fact that a regulator given by the cascade of an oscillatory dynamics, driven by some regulated variables, and of a stabiliser stabilising the cascade of the plant and of the oscillators has the ability of blocking on the steady state of the regulated variables any harmonics matched with the ones of the oscillators. This is the well-celebrated internal model principle. In this paper we are interested to follow the same design route for a controlled plant that is a nonlinear and periodic system with period $T$ : we add a bunch of linear oscillators, embedding $n_{o}$ harmonics that are multiple of $2 \pi / T$, driven by a "regulated variable" of the nonlinear system, we look for a stabiliser for the nonlinear cascade of the plant and the oscillators, and we study the asymptotic properties of the resulting closedloop regulated variable. In this framework the contributions of the paper are multiple: for specific class of minimum-phase systems we present a systematic way of designing a stabiliser, which is uniform with respect to $n_{o}$, by using a mix of high-gain and forwarding techniques; we prove that the resulting closed-loop system has a periodic steady state with period $T$ with a domain of attraction not shrinking with $n_{o}$; similarly to the linear case, we also show that the spectrum of the steady state closed-loop regulated variable does not contain the $n$ harmonics embedded in the bunch of oscillators and that the $L_{2}$ norm of the regulated variable is a monotonically decreasing function of $n_{o}$. The results are robust, namely the asymptotic properties on the regulated variable hold also in presence of any uncertainties in the controlled plant not destroying closed-loop stability.
\end{abstract}

D. Astolfi

Univ Lyon, Université Claude Bernard Lyon 1, CNRS, LAGEPP UMR 5007, 43 boulevard du 11 Novembre 1918, F-69100, Villeurbanne, France

E-mail: daniele.astolfi@univ-lyon1.fr

L. Praly

MINES ParisTech, PSL Research University, CAS - Centre automatique et systémes, Paris 75006, France

L. Marconi

CASY-DEI, University of Bologna, Italy 
Keywords Nonlinear output regulation - Repetitive Control - Minimum Phase Systems $\cdot$ Harmonic rejection

\section{Introduction}

The problem of rejecting or tracking asymptotically periodic or quasi-periodic signals is of primary importance in many applications [29,30,49]. Among them, robotics [25, 41], power electronics [38] and bio-medics engineering [18], just to cite a few. Such a problem is commonly known in control system theory as robust output regulation, see $[11,13,16]$, where the adjective robust refers to the fact that the asymptotic properties are desired to hold not only for the nominal model of the system but also for small perturbations of it. The solution to the robust output regulation problem for finite-dimensional linear time-invariant systems is accredited to Francis, Wonham and Davison who at the same time, but independently, published their main works during the 70's, see, e.g., $[13,16]$. The proposed solution relies on the so-called internal model principle coined by Francis and Wonham in their celebrated work [16], stating that output regulation property is insensitive to plant parameter variations "only if the controller utilises feedback of the regulated variable, and incorporates in the feedback path a suitably reduplicated model of the dynamic structure of the exogenous signals which the regulator is required to process". In turns, if some overall stability properties are guaranteed, the presence of a copy of the exogenous dynamics (also denoted as exosystem) in the regulator provides a "blocking-zero" effect on the desired regulated output at the dynamics excited by such exogenous signals. In other words, the regulated output cannot contain any mode of the exosystem if the overall trajectories are bounded. In practice, an integral action in the controller allows one to achieve zero-DC value of the regulated output, while a given oscillator at a certain frequency, ensures to have zero spectral component at it $[4,19]$.

In our preliminary contribution [4], focused on the problem of nonlinear robust output regulation in presence of periodic exosignals, we have proposed a solution for input-affine nonlinear systems that mimics the aforementioned linear paradigm $[13,16]$. It involves the following two main components.

1. An internal-model unit processing the regulated output which is composed by a bunch of oscillators at a given fundamental frequency and a certain number $n_{o}$, possibly infinite, of its multiples. Such an internal model unit guarantees a blocking-zero property on the regulated error in terms of spectral components, i.e. the regulated output cannot have harmonics at the frequencies embedded in the internal model unit, see [4, Proposition 3] or [19, Proposition 1]. Such a property is insensitive to model perturbations as long as system trajectories are bounded. In doing so, (contrary to large part of nonlinear output regulation theory, see, e.g., $[9,10,33])$ a precise description of the generator of the exosignals is not needed as long as the fundamental period characterising all the spectrum is known. This motivates us in describing the plant dynamics as a $T$-periodic timevarying nonlinear system, without need of a precise description of the exogenous signals dynamics. 
2. A state feedback stabiliser composed by two parts: a preliminary state feedback in charge of stabilising the equilibrium assumed to exist when the exosignals are zero, augmented by a forwarding feedback which serves at stabilising the overall extended dynamics composed by plant and the internal-model unit (see, e.g., [3-5]).

The motivation for such a solution comes from the well-known fact that an inputto-state stable system driven by a periodic input admits (at least locally) periodic solutions of the same period [45, §12]. In our preliminary work [4], however, some important issues were still open. In particular, it was not clear whether the domain of attraction of the steady-state periodic solution shrinks to zero by increasing the dimension of the internal-model unit (i.e. the number of oscillators), and whether asymptotic regulation can be achieved by means of a (countable) infinite-dimensional internal-model (i.e. by using an infinite number of oscillators). We gave a partial answer to this latter question in our second preliminary contribution [5].

The objective of this work is therefore to give an exhaustive answer to both open questions by providing a unifying result and by showing the practical interest of the proposed approach in periodic output regulation frameworks. For this, as in [5], we restrict our attention to the particular class of minimum-phase nonlinear systems, that is, systems possessing a well-defined relative degree with constant high-frequency gain, which are described in normal form (possibly after a change of coordinates), see [24, Chapter 4], and with locally exponentially stable zero-dynamics. This allows us to choose an elementary high-gain feedback as a preliminary stabilising feedback (see, e.g., [46, Lemma 2.2]) and a linear forwarding feedback. In this simplified context, it is shown that the behaviour of the proposed regulator is robust with respect to model uncertainties and uniform in the dimension of the internal model unit (i.e., the number $n_{o}$ of oscillators), in the following sense:

- The high-gain feedback doesn't need to be re-parametrized if the number of oscillators vary. It is chosen before hand, based on the Lipschitz system properties (i.e., the precise knowledge of the plant's dynamics is not needed).

- The proposed regulator ensures boundedness of the overall closed-loop system trajectories and the existence of a (locally) exponentially stable $T$-periodic solution, with a domain of attraction which is uniform in the number of oscillators $n_{o}$, and independent of their frequencies. In other words, a precise knowledge of the period $T$ characterising the plant's dynamics (that is, the period of the exosignals) is not needed to ensure such a boundedness property.

- If the fundamental frequency of the internal-model unit oscillators is selected exactly as the one characterising the plant's dynamics $\frac{2 \pi}{T}$, then the regulated output cannot contain harmonics at the frequencies of such oscillators, and approximate regulation is achieved ${ }^{1}$. Furthermore, the $L_{2}$ norm (over a period) of the steadystate output is inversely proportional to the number of oscillators: the quality of approximate regulation can be therefore improved by augmenting the number of oscillators (and not by increasing the parameters of the high-gain feedback regulator as in high-gain feedback control).

\footnotetext{
1 Note that the notion of approximate considered here is different from the notion of $k$-th order approximate regulation defined in [23], [11, Chapter 2.5].
} 
- If the number of oscillators is infinite, then asymptotic ${ }^{2}$ output regulation is achieved. This infinite dimensional regulator preserves the same bounds in terms of highgain feedback and domain of attraction.

In conclusion, we show that the problem of periodic robust output regulation can be generically solved by an infinite-dimensional internal-model based regulator containing oscillators at all multiples of the basic periodic. Moreover, a good finitedimensional approximation of such a regulator can be obtained by using a finite number of oscillators. In doing so, one obtains a desired accuracy (in terms of $L^{2}$ norm) with bounds of the high-gain term and the domain of attraction which are uniform (i.e., independent) in the desired approximation (i.e., the number of oscillators).

From a technical point of view, the main difficulty to deal with is the fact that each time we modify the dimension of the internal model unit (i.e. we vary the number of oscillators), we have to study a system with different state-space dimension. While most of mathematical tools are well-suited to study the effect of parameter's variations in system dynamics (see, e.g., [27] or [3, Appendix]), it is very hard to compare objects with different dimensions and we are unaware of generic tools developed for this specific purpose. For this reasons, in this work we analyse all the aforementioned properties (all these contributions are new with respect to our preliminary works $[4,5])$ by carefully re-doing all the proofs concerning existence of periodic solutions (which mainly relies on fixed-point theorems) and their stability properties (which mainly relies on Lyapunov analysis), by showing that all these features are uniform in the internal-model system dimension. In such a perspective, the fact of focusing on minimum-phase systems with constant high-frequency gain and unitary relative degree allows us to conceptually simplify most of the (already complex) proofs. The case of higher relative degree can be easily dealt with by means of partial change of coordinates as show in [44]. Details are given in Section 3.5. The case of square multi-input multi-output (i.e. same number of inputs and outputs) systems with constant (and invertible) high-frequency matrix gain is also straightforward and not considered in this work.

This rest of the article is organised as follow. In Section 2 we state the problem formulation and, in Section 3, we provide the main results of this work. With these precise elements at hand, we are in a better position to compare our results with what is available in the literature. This is done in Section 3.4. A numerical example is proposed in Section 4. Conclusions are drawn in Section 5. All proofs are postponed in the Appendix.

Notation. $\mathbb{R}$ is the set of real numbers and $\mathbb{R}_{\geq 0}:=[0,+\infty) ; \mathbb{Z}$ is the set of integers; $\mathbb{N}$ is the set of non-negative integers, and $\mathbb{N}_{>0}$ is the set of positive integers, $\mathbb{C}$ is the set of complex number and $\mathrm{i}=\sqrt{-1}$. Given $x \in \mathbb{C}$, we denote with $\bar{x}$ its conjugate. We denote by $\mathscr{C}^{k}(X ; Y)$ the set of $C^{k}$ functions from $X$ to $Y$, and with $\mathscr{C}_{T}^{k}([0, T] ; X)$ the set of $C^{k} T$-periodic functions from $[0, T]$ to $X$. For compactness, in the following, $\mathscr{C}_{T}^{k}([0, T] ; X)$ will be simply denoted as $\mathscr{C}_{T}^{k}(X)$.

\footnotetext{
2 In this case, exponential stability cannot be anymore guaranteed in view of the presence of an infinite number of poles on the imaginary axis and the use of a bounded (in the sense of [47, Page 24]) control operator. See also $[2,42,43]$ for other examples of such a phenomenon.
} 


\section{Problem Statement}

The objective of this work is to state and prove very precise results concerning the output regulation problem for the particular systems that can be rewritten, under suitable change of coordinates, in the form

$$
\begin{aligned}
& \dot{x}=f(w, x, e) \\
& \dot{e}=q(w, x, e)+u
\end{aligned}
$$

where $(x, e) \in \mathbb{R}^{n} \times \mathbb{R}$ is the state, with $x$ unmeasured $u \in \mathbb{R}$ is the control input, $e \in \mathbb{R}$ is the measured output to be regulated to zero and $w \in \mathbb{R}^{n_{x}}$ are exogenous signals representing references to be tracked or disturbances to be rejected. The number of control problems that can be recast in such a form is very large and examples may be found, for instance, in $[2,9,10,26,31,33]$ and references therein. As discussed in [10, Remark 1], the considered class of systems may look very particular, as it has relative degree 1 between control input $u$ and regulated output e. However, the design methodology described in what follows lends itself to a straightforward extension to systems with higher relative degree [9, eq. (33)]. More details are postponed to Section 3.5. In this work, we suppose that $w$ is $T$-periodic, in other words $w$ is a sufficiently smooth function fulfilling $w(t+T)=w(t)$. To simplify our notations, throughout the rest of this paper we will replace $w$ by $t$ and we will assume that the functions $f, q$ satisfies

$$
f(t+T, x, e)=f(t, x, e), \quad q(t+T, x, e)=q(t, x, e)
$$

for any $t$. We are interested, moreover, in systems which are strongly minimum-phase. In particular, we suppose that when $e=0$, the system

$$
\dot{x}=f(t, x, 0)
$$

admits a unique periodic solution $x_{0}(\cdot)$ which is exponentially stable with some domain of attraction. Since, in what follows, the knowledge of $x_{0}$ is not required and $x$ is not accessible, there is no loss of generality in assuming that $x_{0}(t)$ is the origin of the coordinates for $x$ at time $t$. This allows us to formulate our output regulation problem for the following class of systems

$$
\begin{aligned}
\dot{x} & =f(t, x, e) \\
\dot{e} & =q(t, x, e)+u
\end{aligned}
$$

where $f: \mathbb{R} \times \mathbb{R}^{n} \times \mathbb{R} \rightarrow \mathbb{R}^{n}$ and $q: \mathbb{R} \times \mathbb{R}^{n} \times \mathbb{R} \rightarrow \mathbb{R}$ are $C^{2}$ and $T$-periodic in their first argument, $f$ is such that $f(t, 0,0)=0$ for all $t \geq 0$ and the function $q$ satisfies $\sup _{t \in[0, T]}|q(t, 0,0)|>0$ (otherwise the problem would trivially boil down to a stabilisation context that would be solved, in a semi-global context, by a simple high-gain feedback $u=-\sigma e$, with $\sigma>0$ sufficiently large, see, e.g., [46]).

Our approximate output regulation objective is

$$
\limsup _{t \rightarrow \infty}|e(t)| \leq \mathbf{e}_{p}
$$

with $\mathbf{e}_{p}$ arbitrarily chosen. 
Evidently, a simple way to achieve such a practical regulation property could be that of implementing a high-gain controller of the form $u=-\sigma e$, with $\sigma>0$ large enough, see, e.g., [46, Example 2.1]. The drawback of this controller is that $\sigma$ is the only tunable parameter and it has the undesirable property of amplification of possible (high-frequency) measurement noise, thus being unsuited in practical applications. The regulator we propose incorporates also a tunable internal model allowing to satisfy (2) but also stronger properties that a standard high-gain controller could not achieve. Also, as explained in the introduction, we want a result as less dependent on $f$ and $q$ as possible. The aim of this weak dependency is to make the property (2) robust to uncertainties in $f$ and $q$. This is achieved by asking not the exact knowledge of the pair $(f, q)$ but only that it belongs to a family. Precisely, say that we have a model pair $\left(f_{m}, q_{m}\right)$. We define from it the set of bounding functions

$$
\begin{aligned}
& \sup _{(t, x, e) \in \mathscr{S}_{T}(\mathbf{x}, \mathbf{e})}\left|f_{m}(t, x, e)\right| \leq \mathbf{f}(\mathbf{x}, \mathbf{e}), \sup _{(t, x, e) \in \mathscr{S}_{T}(\mathbf{x}, \mathbf{e})}\left|\frac{\partial f_{m}}{\partial e}(t, x, e)\right| \leq \mathbf{f}_{e}(\mathbf{x}, \mathbf{e}), \\
& \sup _{(t, x, e) \in \mathscr{S}_{T}(\mathbf{x}, \mathbf{e})}\left|\frac{\partial^{2} f_{m}}{\partial x \partial x}(t, x, e)\right| \leq \mathbf{f}_{x x}(\mathbf{x}, \mathbf{e}), \quad \sup _{(t, x, e) \in \mathscr{S}_{T}(\mathbf{x}, \mathbf{e})}\left|\frac{\partial^{2} f_{m}}{\partial e \partial x}(t, x, e)\right| \leq \mathbf{f}_{e x}(\mathbf{x}, \mathbf{e}), \\
& \sup _{(t, x, e) \in \mathscr{S}_{T}(\mathbf{x}, \mathbf{e})}\left|q_{m}(t, x, e)\right| \leq \mathbf{q}(\mathbf{x}, \mathbf{e}), \sup _{(t, x, e) \in \mathscr{S}_{T}(\mathbf{x}, \mathbf{e})}\left|\frac{\partial q_{m}}{\partial e}(t, x, e)\right| \leq \mathbf{q}_{e}(\mathbf{x}, \mathbf{e}), \\
& \sup _{(t, x, e) \in \mathscr{S}_{T}(\mathbf{x}, \mathbf{e})}\left|\frac{\partial q_{m}}{\partial x}(t, x, e)\right| \leq \mathbf{q}_{x}(\mathbf{x}, \mathbf{e}), \sup _{(t, x, e) \in \mathscr{S}_{T}(\mathbf{x}, \mathbf{e})}\left|\frac{\partial q_{m}}{\partial t}(t, x, e)\right| \leq \mathbf{q}_{t}(\mathbf{x}, \mathbf{e}) .
\end{aligned}
$$

where we have introduced the sets

$$
\begin{array}{rlrl}
\mathscr{S}_{x}(\mathbf{x}) & :=\{x:|x| \leq \mathbf{x}\}, & \mathscr{S}_{e}(\mathbf{e}):=\{x:|e| \leq \mathbf{e}\} \\
\mathscr{S}(\mathbf{x}, \mathbf{e}):=\mathscr{S}_{x}(\mathbf{x}) \times \mathscr{S}_{e}(\mathbf{e}), & \mathscr{S}_{T}(\mathbf{x}, \mathbf{e}):=[0, T] \times \mathscr{S}_{x}(\mathbf{x}) \times \mathscr{S}_{e}(\mathbf{e}),
\end{array}
$$

Then we forget $\left(f_{m}, q_{m}\right)$ and instead consider the families of functions $\mathscr{F}$ and $\mathscr{Q}$ defined as follows.

Definition 1 (Family $\mathscr{F}$ ) Given a triplet of positive numbers $\bar{P}_{x}, \underline{P}_{x}, \alpha>0$ and a pair of positive numbers $\mathbf{x}, \mathbf{e}>0$, we say that the function $f: \mathbb{R}_{\geq 0} \times \mathbb{R}^{n} \times \mathbb{R} \mapsto \mathbb{R}^{n}$ belongs to the family $\mathscr{F}\left(\bar{P}_{x}, \underline{P}_{x}, \alpha, \mathbf{x}, \mathbf{e}\right)$ if the following statements holds.

- The function $f$ is $C^{2}, T$-periodic in the first argument and satisfies the following set of inequalities

$$
\begin{aligned}
& \sup _{(t, x, e) \in \mathscr{S}_{T}(\mathbf{x}, \mathbf{e})}|f(t, x, e)| \leq \mathbf{f}(\mathbf{x}, \mathbf{e}), \quad \sup _{(t, x, e) \in \mathscr{S}_{T}(\mathbf{x}, \mathbf{e})}\left|\frac{\partial f}{\partial e}(t, x, e)\right| \leq \mathbf{f}_{e}(\mathbf{x}, \mathbf{e}), \\
& \sup _{(t, x, e) \in \mathscr{S}_{T}(\mathbf{x}, \mathbf{e})}\left|\frac{\partial^{2} f}{\partial x \partial x}(t, x, e)\right| \leq \mathbf{f}_{x x}(\mathbf{x}, \mathbf{e}), \sup _{(t, x, e) \in \mathscr{S}_{T}(\mathbf{x}, \mathbf{e})}\left|\frac{\partial^{2} f}{\partial e \partial x}(t, x, e)\right| \leq \mathbf{f}_{e x}(\mathbf{x}, \mathbf{e}) \text {. } \\
& \sup _{(t, x, e) \in \mathscr{S}_{T}(\mathbf{x}, \mathbf{e})}|f(t, x, e)| \leq \mathbf{f}(\mathbf{x}, \mathbf{e}), \quad \sup _{(t, x, e) \in \mathscr{S}_{T}(\mathbf{x}, \mathbf{e})}\left|\frac{\partial f}{\partial e}(t, x, e)\right| \leq \mathbf{f}_{e}(\mathbf{x}, \mathbf{e}), \\
& \sup _{(t, x, e) \in \mathscr{S}_{T}(\mathbf{x}, \mathbf{e})}\left|\frac{\partial^{2} f}{\partial x \partial x}(t, x, e)\right| \leq \mathbf{f}_{x x}(\mathbf{x}, \mathbf{e}), \sup _{(t, x, e) \in \mathscr{S}_{T}(\mathbf{x}, \mathbf{e})}\left|\frac{\partial^{2} f}{\partial e \partial x}(t, x, e)\right| \leq \mathbf{f}_{e x}(\mathbf{x}, \mathbf{e}) .
\end{aligned}
$$


- There exists a $T$-periodic $C^{1}$ positive definite matrix $P_{x}: \mathbb{R}_{\geq 0} \rightarrow \mathbb{R}^{n \times n}$, satisfying

$$
\begin{gathered}
0<\underline{P}_{x} I \leq P_{x}(t) \leq \bar{P}_{x} I, \\
\dot{P}_{x}(t)+P_{x}(t) \frac{\partial f}{\partial x}(t, 0,0)+\frac{\partial f^{\top}}{\partial x}(t, 0,0) P_{x}(t) \leq-2 \alpha P_{x} .
\end{gathered}
$$

Definition 2 (Family $\mathscr{Q}$ ) Given a pair of positive numbers $\mathbf{x}, \mathbf{e}>0$, we say that the function $q: \mathbb{R}_{\geq 0} \times \mathbb{R}^{n} \times \mathbb{R} \mapsto \mathbb{R}^{n}$ belongs to the family $\mathscr{Q}(\mathbf{x}, \mathbf{e})$ if it is $C^{2}$, T-periodic in its first argument and satisfies the following set of inequalities

$$
\begin{aligned}
\sup _{(t, x, e) \in \mathscr{S}_{T}(\mathbf{x}, \mathbf{e})}|q(t, x, e)| & \leq \mathbf{q}(\mathbf{x}, \mathbf{e}), \sup _{(t, x, e) \in \mathscr{S}_{T}(\mathbf{x}, \mathbf{e})}\left|\frac{\partial q}{\partial e}(t, x, e)\right| \leq \mathbf{q}_{e}(\mathbf{x}, \mathbf{e}), \\
\sup _{(t, x, e) \in \mathscr{S}_{T}(\mathbf{x}, \mathbf{e})}\left|\frac{\partial q}{\partial x}(t, x, e)\right| & \leq \mathbf{q}_{x}(\mathbf{x}, \mathbf{e}), \sup _{(t, x, e) \in \mathscr{S}_{T}(\mathbf{x}, \mathbf{e})}\left|\frac{\partial q}{\partial t}(t, x, e)\right| \leq \mathbf{q}_{t}(\mathbf{x}, \mathbf{e}) .
\end{aligned}
$$

Note that systems of the form (1) satisfying $f, q \in \mathscr{F}, \mathscr{Q}$ are typically obtained when deriving a normal form in presence of smooth periodic reference to be tracked or perturbation to be rejected. See, for instance, $[2,26,31]$ and references therein.

Remark Note that in light of (4), (5), the set $\mathscr{F}\left(\bar{P}_{x}, \underline{P}_{x}, \alpha, \mathbf{x}, \mathbf{e}\right)$ characterises functions for which the zero dynamics of (1), namely $\dot{x}=f(t, x, 0)$, is locally exponentially stable, with a given decreasing rate $-\alpha$. Indeed, the function $V(t, x)=x^{\top} P_{x}(t) x$ can be used as Lyapunov function to establish such stability properties. Since in this work we are not interested in establishing semi-global results, the properties (4) and (5) will be the only assumptions made on the zero-dynamics (1a). Such assumptions are indeed milder than those commonly stated in semi-global output regulation results, where typically the zero-dynamics (1a) is asked to be input-to-state stable (in short, ISS) or integral ISS (in short, iISS) with respect to $e$, see, e.g., [10, 26, 44, 50].

\section{Main Results}

\subsection{Internal-Model Based Regulator Design}

The feedback law we propose is made of two sets of tunable parameters:

- an integer $n_{o} \in \mathbb{N}$ and two positive real numbers $\sigma, \mu>0$;

- two sequences of positive real numbers $n_{z \ell}$ and $\omega_{\ell}$ satisfying

$$
\begin{array}{rlrl}
\sum_{\ell=0}^{\infty} n_{z \ell}=\bar{N}_{z}^{2} & <+\infty, & \\
n_{z(\ell+1)} & <n_{z \ell} & \forall 0 \leq \ell, \\
\ell n_{z \ell} & \leq m n_{z m} & \forall(\ell, m): 0<m \leq \ell, \\
\ell^{2} n_{z \ell} \leq m^{2} n_{z m} & \forall(\ell, m): 0 \leq \ell \leq m, \\
\omega_{\ell}=\ell \widehat{\omega} & \forall \ell>0,
\end{array}
$$


for some $\widehat{\omega}>0$. Actually, as an illustration or for more specificity, we consider often the particular case

$$
\begin{aligned}
& n_{z 0}=2, \\
& n_{z \ell}=\frac{1}{\ell^{1+\varepsilon}}, \quad \forall \ell \in \mathbb{N}_{>0} \quad \varepsilon \in(0,1] .
\end{aligned}
$$

The proposed dynamic controller takes the form

$$
\begin{aligned}
\dot{z} & =\Phi z+\Gamma e \\
u & =-\sigma e+\mu M^{\top} N_{z}(z-M e),
\end{aligned}
$$

where $z=\left(z_{0}, \ldots, z_{n_{o}}\right) \in \mathbb{R}^{2 n_{o}+1}$, is the state of the controller and the matrices $\Phi, N_{z} \in$ $\mathbb{R}^{\left(2 n_{o}+1\right) \times\left(2 n_{o}+1\right)}$ and $\Gamma, M \in \mathbb{R}^{2 n_{o}+1}$ are defined as

$$
\begin{array}{ll}
\Phi:=\operatorname{blkdiag}\left(0, \Phi_{1}, \ldots, \Phi_{n_{o}}\right), & \Phi_{\ell}=\left(\begin{array}{cc}
0 & \omega_{\ell} \\
-\omega_{\ell} & 0
\end{array}\right), \\
N_{z}=\operatorname{blkdiag}\left(n_{z 0}, N_{z 1}, \ldots, N_{z n_{o}}\right), & N_{z \ell}=n_{z \ell} I_{2} \quad \forall \ell=1, \ldots, n_{o}, \\
M=\left(1, M_{1}^{\top}, \ldots, M_{n_{o}}^{\top}\right)^{\top}, & M_{\ell}=(1,0)^{\top} \quad \forall \ell=1, \ldots, n_{o}, \\
\Gamma=-(\Phi+\sigma I) M . &
\end{array}
$$

It can be readily seen from the definition of (10), that the regulator (9) is composed of two parts: an internal-model unit (9a), that is, the $z$-dynamics, characterised by an integrator and a bunch of linear oscillators at frequencies $\omega_{\ell}$, and a linear stabilizing term (9b) having embedded the high-gain feedback law $-\sigma e$ needed for stability purposes (see, e.g., $[33,46])$. As shown in the rest of the paper, the feedback law (9) guarantees that (2) holds with any (arbitrarily small) $\mathbf{e}_{p}$ chosen a priori and independent of $n_{o}$, and moreover

$$
\lim _{n_{o} \rightarrow+\infty} \limsup _{t \rightarrow+\infty}|e(t)|=0,
$$

when the basic frequency characterizing the oscillators $\Phi_{\ell}$ of the internal-model unit is selected as $\widehat{\omega}=\frac{2 \pi}{T}$, that is $\omega_{\ell}=\ell \frac{2 \pi}{T}$ for any $\ell=1, \ldots, n_{o}$.

\subsection{Approximate Regulation}

First, we study the interconnection of the finite-dimensional controller (9) in closedloop with the system (1) and we establish a certain number of properties concerning the existence and stability of a steady-state trajectory and the norm of the corresponding regulated output. The proof of the following theorem is postponed to Section A.

Theorem 1 Given a triplet $\left(\bar{P}_{x}, \underline{P}_{x}, \alpha\right)$ and any fixed $\mu \geq 1$, there exist positive real numbers $\mathbf{x}, \mathbf{e}, \mathbf{z}, \sigma^{\star}$, independent of $n_{o}$, such that, the closed-loop system (1), (9), with any $f \in \mathscr{F}\left(2 \mathbf{x}, 2 \mathbf{e}, \bar{P}_{x}, \underline{P}_{x}, \alpha\right)$ and any $q \in \mathscr{Q}(2 \mathbf{x}, 2 \mathbf{e})$, satisfies the following statements: 
1) for any $\sigma>\sigma^{\star}$ and any $n_{o}>0$, the closed-loop system (1), (9) admits a $T$ periodic solution $\left(x_{p}, e_{p}, z_{p}\right) \in \mathscr{C}_{T}^{2}\left(\mathbb{R}^{n} \times \mathbb{R} \times \mathbb{R}^{2 n_{o}+1}\right)$ satisfying

$$
\sup _{t \in[0, T]}\left|x_{p}(t)\right| \leq \mathbf{x}, \quad \sup _{t \in[0, T]}\left|e_{p}(t)\right| \leq \mathbf{e}, \quad \sup _{t \in[0, T]} \sqrt{z_{p}(t)^{\top} N_{z} z_{p}(t)} \leq \mathbf{z} .
$$

Moreover $\left(x_{p}, e_{p}, z_{p}\right)$ is locally exponentially stable with a domain of attraction that includes the set

$$
\mathscr{N}(\mathbf{x}, \mathbf{e}, \mathbf{z})=\left\{(x, e, z):|x| \leq 2 \mathbf{x},|e| \leq 2 \mathbf{e}, \sqrt{z^{\top} N_{z} z} \leq 2 \mathbf{z}\right\}
$$

which is independent of $n_{o}$.

2) There exists $\psi_{1}, \psi_{x}>0$, independent of $n_{o}, \sigma, \mu$, such that, for any $\sigma>\sigma^{\star}$ and any $n_{o}>0$, the corresponding $T$-periodic solution $\left(x_{p}, e_{p}, z_{p}\right)$ of the closed-loop system (1), (9) established in item 1) satisfies

$$
\sup _{t \in[0, T]}\left|e_{p}(t)\right| \leq \frac{\psi_{1}}{\sigma}, \quad \sup _{t \in[0, T]}\left|x_{p}(t)\right| \leq \frac{\psi_{x}}{\sigma} .
$$

3) Let $\sigma>\sigma^{\star}$ and $n_{o}$ be fixed. If, for some $\ell$ in $\left\{1, \ldots, n_{o}\right\}$, there exists an integer $\mathfrak{K}_{\ell}>0$ such that $\omega_{\ell}$ in (9), satisfies

$$
\omega_{\ell}=\mathfrak{K}_{\ell} \frac{2 \pi}{T},
$$

then the corresponding T-periodic solution $\left(x_{p}, e_{p}, z_{p}\right)$ of the closed-loop system (1), (9) established in item 1) satisfies

$$
\int_{0}^{T} \sin \left(\mathfrak{K}_{\ell} \frac{2 \pi}{T} t\right) e_{p}(t)=\int_{0}^{T} \cos \left(\mathfrak{K}_{\ell} \frac{2 \pi}{T} t\right) e_{p}(t)=0 .
$$

4) Suppose that we have $\widehat{\omega}=\frac{2 \pi}{T}$ in (7e). Then, there exists $\psi_{2}>0$, independent of $n_{o}, \sigma, \mu$, such that, for any $\sigma>\sigma^{\star}$ and $n_{o}>0$, the corresponding $T$-periodic solution $\left(x_{p}, e_{p}, z_{p}\right)$ of the closed-loop system (1), (9) established in item 1) satisfies

$$
\begin{gathered}
\int_{0}^{T}\left|e_{p}(t)\right|^{2} d t \leq \frac{\psi_{2}}{\left(n_{o}+1\right)^{2}}, \\
\int_{0}^{T}\left(\begin{array}{c}
\cos \left(\omega_{\ell} t\right) \\
\sin \left(\omega_{\ell} t\right)
\end{array}\right) e_{p}(t) d t=0 \quad \forall \ell \in\left\{1, \ldots, n_{o}\right\} .
\end{gathered}
$$

Theorem 1 establishes several properties about the solutions of the closed-loop system (1), (9). Item 1) states that for $\sigma$ large enough, the closed-loop system admits an exponentially stable $T$-periodic steady-state trajectory. Existence, stability and domain of attraction of such a steady-state is robust to model uncertainties and independent of the parameters of the internal-model unit in (9), that it is independent of $n_{o}$, and the sequences $n_{z \ell}, \omega_{\ell}$ characterising the frequencies of the oscillators of the $z$-dynamics, provided the conditions (7) hold. Furthermore, it is shown that the initial condition $z(0)=0$ for the internal-model unit (9) is always a "good initial solution" as it is always contained in the domain of attraction (13). 
Item 2 establishes that the controller (9) preserves the high-gain property of a feedback without internal-model: the infinity norm of the steady-state trajectory of the regulated output $e$ can be arbitrarily made small by augmenting the parameter $\sigma$, see (14). In other words, we don't loose the properties of a simple high-gain feedback $u=-\sigma e$. Moreover, such a high-gain property is robust to model uncertainties and independent of the parameters $n_{z \ell}, \omega_{\ell}$ in (7).

Item 3 characterises the behaviour of the steady-state of the regulated output $e$ when the frequency of one oscillator is a multiple of the basic frequency $\frac{2 \pi}{T}$ characterising the periodicity of the frequencies $f, q$. In this case, the Fourier coefficient of $e$ corresponding to that frequency is zero. Such a property evidently suggests a strategy to select the parameters $\omega_{\ell}$ in (9) when the periodicity $T$ of the functions $f, q$ is known. This is well established in item 4.

In particular, the inequality (16) shows that the $L_{2}$ norm of the steady-state regulated output $e$ can be made arbitrarily small by augmenting the number of oscillators, if those are chosen so that their frequency is multiple of the basic frequency $\frac{2 \pi}{T}$. This is a consequence of the fact that each corresponding Fourier coefficient is zero, as established by (15). Note that although a similar result were already proved in [4], [19], the novelty of item 4) is that here we are able to show that the inequality (16) is uniform in the parameters of the controller (9). This implies that, from a practical point of view, one can first fix the parameters $\mu, \sigma$, and then arbitrarily increase $n_{o}$ so that to reduce the $L_{2}$ norm of the regulated output. Furthermore, in doing so, the domain of attraction is guaranteed to always exist and contain a prescribed set of initial conditions independent of the parameters of the internal-model. In other words, when the period $T$ is known, the approximate regulation objective (2) can be satisfied by augmenting the number of oscillators and not the high-gain parameter.

In the next section, we will show that the bound (11) hold when considering the non-implementable infinite-dimensional case, corresponding to the limit case of the regulator (9) in which $n_{o}=+\infty$.

\subsection{Exact Regulation}

In this section we want to study the limit case (non-implementable) in which the period $T$ is perfectly known and the number of oscillators in the regulator (9) is chosen as infinite $n_{o}=\infty$, with $\widehat{\omega}=\frac{2 \pi}{T}$, that is $\omega_{\ell}=\frac{2 \pi}{T}$ for all $\ell \in\{0,1, \ldots, \infty\}$. In particular we aim at establishing that, in such a case, exact regulation is achievable and (11) is satisfied. To this end, let us define the linear operators $\Phi, N_{z}, M, \Gamma$ as

$$
\begin{array}{lll}
\Phi:=\left(\Phi_{\ell}\right)_{\ell \in \mathbb{N}_{0}}, & \Phi_{0}:=0, & \Phi_{\ell}:=\ell \frac{2 \pi}{T}\left(\begin{array}{cc}
0 & 1 \\
-1 & 0
\end{array}\right), \\
N_{z}:=\left(N_{z \ell}\right)_{\ell \in \mathbb{N}_{0}}, & N_{z 0}:=n_{z 0}, & N_{z \ell}:=n_{z \ell}\left(\begin{array}{ll}
1 & 0 \\
0 & 1
\end{array}\right), \\
M:=\left(M_{\ell}\right)_{\ell \in \mathbb{N}_{0}}, & M_{0}:=1, & M_{\ell}:=(1,0)^{\top}, \\
\Gamma:=-(\Phi+\sigma I) M &
\end{array}
$$


with $\left(n_{z \ell}\right)_{\ell \in \mathbb{N}_{\geq 0}}$ being the sequence defined in (7a). We denote with $\mathscr{Z}$ the space of sequences

$$
\mathscr{Z}:=\left\{z=\left(z_{\ell}\right)_{\ell \in \mathbb{N}_{0}}, z_{0} \in \mathbb{R}, z_{\ell} \in \mathbb{R}^{2}, \ell \in \mathbb{N}\right\}
$$

and we define the space $\mathscr{L}_{N_{z}}^{2}$ as

$$
\mathscr{L}_{N_{z}}^{2}:=\left\{z \in \mathscr{Z}:\|z\|_{N_{z}}^{2}:=z^{\top} N_{z} z=\sum_{\ell=0}^{\infty} n_{z \ell}\left|z_{\ell}\right|^{2}<\infty\right\}
$$

with $N_{z}$ being defined in (17). This space, being linearly isometric with the standard $\mathscr{L}^{2}$ space, is complete. In this section, we address the specific case in which the regulator is selected as

$$
\begin{aligned}
\dot{z} & =\Phi z+\Gamma e \\
u & =-\sigma e+\mu M^{\top} N_{z}(z-M e),
\end{aligned}
$$

where $z \in \mathscr{Z}$, is the state with initial condition $z(0) \in \mathscr{L}_{N_{z}}^{2}$, and the linear operators $\Phi, M, N_{z}$ are now defined as in (17). As now the state $z$ is a vector of infinite, but countable dimension, we will consider only solutions in the space $\mathscr{L}_{N_{z}}^{2}$. We have the following result showing that exact regulation can be achieved. The proof of the following theorem is postponed to Section A.

Theorem 2 Let the triplet $\left(\bar{P}_{x}, \underline{P}_{x}, \alpha\right)$ be given and fix any $\mu \geq 1$. Consider the real numbers $\mathbf{x}, \mathbf{e}, \mathbf{z}$ and $\sigma^{\star}$ given by Theorem 1. There exists $\sigma_{\infty}^{\star}<\sigma^{\star}$ such that, for any $\sigma>\sigma_{\infty}^{\star}$, any trajectory $(x(t), e(t), z(t))$ of the closed-loop system (1), (9), with $f \in \mathscr{F}\left(2 \mathbf{x}, 2 \mathbf{e}, \bar{P}_{x}, \underline{P}_{x}, \alpha\right)$ and $q \in \mathscr{Q}(2 \mathbf{x}, 2 \mathbf{e})$, starting in the set

$$
\left\{(x, e, z) \in \mathbb{R}^{n} \times \mathbb{R} \times \mathscr{L}_{N_{z}}^{2}:|x| \leq 2 \mathbf{x},|e| \leq 2 \mathbf{e},\|z\|_{N_{z}} \leq 2 \mathbf{z}\right\},
$$

is defined and complete forward in time, bounded in $\mathbb{R}^{n} \times \mathbb{R} \times \mathscr{L}_{N_{z}}^{2}$, and satisfies $\lim _{t \rightarrow \infty} e(t)=0$ and $\lim _{t \rightarrow \infty} x(t)=0$.

Theorem 2 establishes that with an infinite-dimensional regulator, exact regulation can be achieved if the period $T$ characterising the functions $f, q$ is perfectly known, and if the regulator embeds an infinite-number of oscillators at $\frac{2 \pi}{T}$ and all its multiples. In practice, such a result confirms the property (11) of the finite dimensional regulator (9). It is noticed that in statement we showed that the domain of attraction in terms of $(x, e)$-coordinates is not reduced with respect to those given by Theorem 1. Furthermore, for the same values of $\mathbf{x}, \mathbf{e}$, the resulting high-gain parameter $\sigma$ can be chosen smaller with an exact infinite-dimensional regulator. The main motivation to this fact is that, in the approximate case, the steady-state solution $x_{p}$ does not coincide with the origin, thus reducing the stability margins ensured by (5). In turn, $\sigma$ has to be chosen larger to compensate such a loss. 


\subsection{Literature Review}

The problem of periodic output regulation has been studied in the past decades by many authors with many different tools and ideas. Although various approaches as ours allow to cope with more general dynamics, for the sake of precision, we restrict our discussion to systems which admit a normal form like the one at the beginning of Section 2 or more generally like (21). We revisit the following main approaches based on the use of "smooth regulators".

- Nonlinear output regulation. Starting from the notable results on the so-called non-linear regulator equations [9] in a non-equilibrium context, the development of output regulation theory has been mainly pursued in the context of minimumphase systems of the form (1). The design of internal-models has been focused mainly in the sense of input-cancellation/observation, that is, with the purposes of reproducing the asymptotic behaviour of the zeroing steady-state input $-q(t, 0,0)$ for system (1b), see, e.g., [10,33]. Although these approaches can ensure asymptotic regulation with finite-dimensional regulators, it is not clear whether they can be extended in a non-matching case, i.e. without the use of a normal form (1). Furthermore, as discussed in see [6,7], asymptotic regulation is lost as soon as unstructured model uncertainties are considered. Approximate asymptotic solutions have been also proposed in $[8,17,32]$, but again, the extension to more general classes of systems (as in [4]) is not clear.

- Repetitive control. Based on the fact that a delay can be used as a universal generator of periodic signal, the repetitive control approach was first proposed at the end of the 80's, [22] for linear systems in order to solve periodic output regulation problems, with remarkable results in the context of discrete-time systems [30] and practical applications [29]. Nonlinear extensions were proposed for instance in $[2,19,38]$. Similarly to our approach, finite-dimensional approximations based on Fourier approximation were used in [19], based on the fact that a delay can be equivalently represented by an infinite-number of oscillators ${ }^{3}$ In this spirit, our work contributes also to the repetitive control theory clarifying the uniformity aspects in terms of basin of attraction of periodic solutions with respect to the approximation of the internal-model unit.

- Adaptive learning control. An approach similar to repetitive control is also the one denoted as adaptive learning control, see, e.g., [15, 34, 48], developed in the context of minimum-phase systems (1), with the objective of estimating the Fourier coefficients of the zeroing steady-state input $-q(t, 0,0)$ [15], or canceling it by means of delays $[34,48]$. Extensions to systems not possessing a normal forms and practical implementations issues related to the delay (and therefore the asymptotic properties of a discretised regulator) have not been discussed.

- Input disturbance observers. Finally, for systems in normal form, input disturbance observers can be used in output regulation of minimum-phase systems (1), see, e.g., [21]. Again, the extension of such an approach to more general classes of systems (as in [4]) is not clear.

\footnotetext{
3 This can be shown, for instance, by using Riesz bases, see Example 2.6.12 in [47].
} 
3.5 Higher-Relative Degree Case via Partial-State Feedback

Consider now a system of relative degree higher than one and described by

$$
\begin{aligned}
& \dot{\chi}=f_{0}\left(t, \chi, \xi_{1}\right) \\
& \dot{\xi}_{i}=\xi_{i+1} \quad i=1, \ldots, r-1, \\
& \dot{\xi}_{r}=q_{0}(t, \chi, \xi)+u
\end{aligned}
$$

with $\chi \in \mathbb{R}^{n}, \xi=\left(\xi_{1}, \ldots, \xi_{r}\right)^{\top} \in \mathbb{R}^{n}$, and suppose that our output regulation objective is now given by

$$
\limsup _{t \rightarrow \infty}\left|\xi_{1}(t)\right| \leq \boldsymbol{\xi}_{1 p} .
$$

Following for instance [44], we consider the change of coordinates

$$
\xi_{r} \mapsto e:=\xi_{r}+\sum_{i=1}^{r-1} a_{i} \xi_{i}
$$

where $a_{i}$ are chosen so that $\lambda^{r-1}+a_{1} \lambda^{r-2}+\ldots+a_{r_{2}} \lambda+a_{r-1}$ is a Hurwitz polynomial. In the new coordinates, system (21) reads as

$$
\begin{aligned}
& \dot{x}=f(t, x, e) \\
& \dot{e}=q(t, x, e)+u
\end{aligned}
$$

with $x:=\left(\chi^{\top}, y^{\top}\right)^{\top}, y:=\left(\xi_{1}, \ldots, \xi_{r-1}\right)^{\top}$,

$$
\begin{aligned}
f(t, x, e) & :=\left(\begin{array}{c}
f_{0}(t, \chi, C y) \\
A y+B e
\end{array}\right) \\
A & :=\left(\begin{array}{cc}
0_{r-2,1} & I_{r-2} \\
-a_{1}-a_{2} \cdots-a_{r-1}
\end{array}\right), \quad B:=\left(\begin{array}{c}
0_{r-2,1} \\
1
\end{array}\right), \quad C:=\left(\begin{array}{ll}
1 & 0_{1, r-1}
\end{array}\right), \\
q(t, x, e) & :=q_{0}\left(t, \chi, \xi_{1}, \ldots, \xi_{r-1}, e-\sum_{i=1}^{r-1} a_{i} \xi_{i}\right)+\sum_{i=1}^{r-2} a_{i} \xi_{i+1}+a_{r-1}\left(e-\sum_{i=1}^{r-1} a_{i} \xi_{i}\right) .
\end{aligned}
$$

By construction, if $f_{0}, q_{0}$ are $C^{2}$ and $T$-periodic, so are $q$ and $f$. As a consequence, we can apply the control design proposed in Sections 3.2, 3.3 to system (23). In particular, concerning the result of Theorem 1, it is straightforward to see that the control law (9) applies to system (23) insuring the desired properties for the new regulated output $e$ of system (23). Then, by linearity of the change of coordinates, the properties on $y, e$ can be used to analyse the behaviour of $\xi_{1}$. Indeed, the $r$-th derivative of $\xi_{1}$, i.e., $\xi_{1}^{(r)}$, is given by

$$
\xi_{1}^{(r)}=-\sum_{k=1}^{r-1} a_{k} \xi_{1}^{(r-1-k)}+e .
$$

With $\lambda^{r-1}+a_{1} \lambda^{r-2}+\ldots+a_{r_{2}} \lambda+a_{r-1}$ being a Hurwitz polynomial, we have

$$
\limsup _{t \rightarrow \infty}\left|\xi_{1}(t)\right| \leq \kappa_{\infty} \mathbf{e}_{p} \quad, \quad \int_{0}^{T} \xi_{1}(t)^{2} d t \leq \kappa_{2} \int_{0}^{T} e(t)^{2} d t
$$


where the real numbers $k_{\infty}$ and $k_{2}$ depends only on the $a_{k}$. The regulation objective (22) is then satisfied by selecting $\mathbf{e}_{p}<\frac{\boldsymbol{\xi}_{1 p}}{k_{\infty}}$.

A formal theorem concerning the design of a regulator for the output regulation problem for system (21) is therefore not given as it can be directly inherited by combining the computations of this section and the results of Theorems 1 and 2.

\section{Example}

\subsection{Linear Bode Analysis}

In this section we provide a numerical example to corroborate the theoretical results of Section 3. First, in order to have a deeper insight of the proposed algorithm, we consider a simple linear case with no zero-dynamics given by

$$
\dot{e}=u+q(t)
$$

and we analyse the difference between the transfer functions of a high-gain feedback regulator

$$
u=-\sigma e
$$

and the internal model based regulator (9). Figure 1 shows the transfer function between the input $q$ and the output $e$ for the closed-loop systems (24), (25), respectively (24), (9), when $\sigma=2$ and the other parameters are selected as $\mu=1, n_{z \ell}$ selected as in (8) with $\varepsilon=0.5, \widehat{\omega}=2 \pi$, and $n_{o}=10$. As expected, it is readily seen that the effect of the internal model is to add blocking zeros at the desired frequencies $\ell \widehat{\omega}$, $\ell=0,1, \ldots, 10$, while preserving the same transfer function of the high-gain feedback (25) at higher frequencies. In the next section, we will show in a numerical simulation that this blocking effect is preserved in the nonlinear context, confirming the results of Theorems 1 and 2.

\subsection{Numerical Example}

Consider here as a simple example a system with unitary relative degree of the form (1). For the simulations, the nominal functions $f, q$ are selected as

$$
\begin{aligned}
& f(t, x, e)=\left(\begin{array}{c}
-\frac{1}{5} x_{1}+\sqrt{3} x_{2}+\frac{1}{10} \sin \left(x_{2}\right) \\
-\sqrt{3} x_{1}-x_{2}+\frac{1}{10} x_{2}^{2}
\end{array}\right)+\left(\begin{array}{c}
0 \\
x_{2} e
\end{array}\right)+\left(\begin{array}{c}
\sin (2 \pi t) \\
\cos (4 \pi t)(1+\sin (2 \pi t))
\end{array}\right), \\
& q(t, x, e)=1+x_{1}+\arctan \left(e x_{2}\right)+\sum_{k=1}^{4} \cos ^{k}(2 \pi t)
\end{aligned}
$$

The functions $f, q$ have been randomly chosen "ugly" so that other frameworks for asymptotic regulation such as $[10,33]$ cannot be explicitly applied. It is readily seen that, around the origin, the $x$-dynamics is locally exponentially stable, while for large values of $x$, it is unstable due to the term $x_{2}^{2}$. Furthermore, $f, q$ are $T$-periodic smooth functions with period $T=1$. Hence, the $f, q$ are included in the Families $\mathscr{F}$ and $\mathscr{Q}$ of Definitions 1 and 2. 
We then evaluated the steady-state performances of a simple high-gain feedback (25), and the internal model based regulator (9), in two scenarios: without any measurement noise, and in presence of high-frequency measurement noise $v$. In the noisy scenario, the high-gain feedback (25) becomes

$$
u=-\sigma(e+v)
$$

while the internal model based regulator (9) reads as

$$
\begin{aligned}
\dot{z} & =\Phi z+\Gamma(e+v) \\
u & =-\sigma e+\mu M^{\top} N_{z}[z-M(e+v)] .
\end{aligned}
$$

We then performed different simulations. Since we are not interested in transient response, the initial conditions are selected always as $x(0)=(1,-2), e(0)=4$ and $z(0)=0$. For the high-gain feedback, we selected different values of $\sigma=\{2,10,20,40\}$. For the controller (9) we selected $\sigma=2, \mu=1$ and $n_{z \ell}$ selected as in (8) with $\varepsilon=0.5$ The number of oscillators is varied in the range $\{0, \ldots, 4\}$, with 0 corresponding to a pure integral action, while the basic frequency $(7 \mathrm{e})$ is selected as $\widehat{\omega}=\left\{\frac{2 \pi}{T}, 0.99 \frac{2 \pi}{T}, 0.95 \frac{2 \pi}{T}, \frac{2 \pi}{T} \varphi_{g}\right\}$ with $\varphi_{g}=\frac{1+\sqrt{5}}{2}$ being the golden number. Simulations have been performed with Matlab-Simulink, with a fixed-step time algorithm, sampling time $10^{-5}$, over a time length of 150 seconds.

As established in Theorem 1, convergence to a steady-state trajectory $e_{p}$ is guaranteed no matter the dimension $n_{o}$ and the frequency $\hat{\omega}$. Table 1 lists the values of the $L_{\infty}$ norm and $L_{2}$ norm of the steady-state error $e_{p}$ with the high-gain feedback (25) for the different values of $\sigma$. Applying a FFT (fast Fourier transform) on the last 20 seconds of simulations (so that solutions reached their steady-state) we identified the corresponding main frequencies Figure 2 of the steady-state error $e_{p}$ for $\sigma=2$, showing that the main Fourier coefficients are at frequencies $2 k \pi$, with $k=0,1, \ldots, 4$, as expected since $T=1$. Then, Table 2 lists the values of the $L_{\infty}$ norm and $L_{2}$ norm of the steady-state error $e_{p}$ for the controller (9) in the different scenarios. The corresponding Fourier coefficients for $\widehat{\omega}=\{1,0.99,0.95\} \frac{2 \pi}{T}$ are shown in Figures 3, 4 and 5 . We can observe that when the basic frequency $\widehat{\omega}$ is less accurate, the errors on the higher 3-4 frequencies becomes more important and therefore the blocking effect of the zero becomes less useful. As a consequence, when the frequency is not perfectly known, it may be not so interesting to put only multiple frequencies of $\widehat{\omega}$.

From Tables 1 and 2, it is immediately seen the remarkable increase of performances (in terms of steady-state $L_{\infty}$ and $L_{2}$ norms) with the proposed controller, even with a very bad knowledge of $T$, with respect to a pure high-gain feedback: for the same value of $\sigma$, we obtain a sensible reduction of both norms when $\widehat{\omega}=\frac{2 \pi}{T} \varphi_{g}$, and to have the similar performances with a high-gain feedback we should take a much higher value of $\sigma$. However, note that when $\hat{\omega}$ is taken very distant from the nominal value $\frac{2 \pi}{T}$, adding extra oscillators is not very useful: indeed the sup and $L_{2}$ norms are not reduced anymore. On the contrary, when $T$ is perfectly known, with only 4 oscillators we are already able to achieve almost perfect tracking as the remaining error is nearly negligible.

Finally, the same scenarios have been performed in presence of high-frequency measurement noise $v$ generated by colouring some random white noise with a highpass filter. In simulations we used a Simulink "Band-Limited White Noise" block 
with noise power $10^{-3}$ and sampling time $10^{-5}$ and the following transfer function

$$
H(s)=\frac{s^{2}}{s^{2}+3 s+2}
$$

with $s$ being the Laplace operator. Simulations show that $\sup _{t \in[0, \infty)}|v(t)| \leq 40$. The values of the $L_{\infty}$ and $L_{2}$ norms (computed over one random period $T$ among the last 30 seconds of simulations) of the asymptotic value of the error are listed in Tables 1 and 2. Again, simulations confirm the advantages (in term of $L_{\infty}$ and $L_{2}$ norms) of the proposed internal model based regulator (9) over a high-gain feedback controller (25).

\section{Conclusions}

In this work we addressed the problem of exact and approximate periodic robust output regulation for minimum-phase systems. We investigated the use of an internalmodel based regulator which is a straightforward extension of the linear case established by Francis, Wonham and Davison, and conceptually similar to what is used in practical repetitive control scheme approaches. In practice, the internal model unit is composed by a bunch of linear oscillators processing the regulated output.

The main contribution of this work is to establish that the domain of attraction of an exponentially stable periodic solution is uniform in the parameters characterising the proposed regulator (i.e. the number of oscillators and their frequencies). The result is also robust to model uncertainties as only the Lipschitz properties of the system dynamics (and not the exact expressions) are used in the computations. Is is shown that the quality (in terms of $L_{2}$ norm) of the periodic steady-state of the regulated error improves by augmenting the number of oscillators and that exact regulation can be achieved when the number of oscillator is infinite and the period is perfectly known. Simulations confirm the theoretical findings and shows the improvements of the proposed regulator with respect to a pure high-gain feedback controller or a simple PI controller.

The main results of this work shows that the best performances with the proposed regulator can be obtained when the period characterising the periodicity of disturbances/references is perfectly known. This consideration leads to many open questions concerning the knowledge of the frequencies of perturbations/references in practical applications, optimal choices of the parameters of the regulator in terms of number of oscillators and their frequencies, and possible strategies for offline/online identification of such frequencies. For all these problems, adaptive, identification or learning techniques may be a key tool, see $[1,8,17,28,35,36,40,44,50]$ as few examples.

Finally, from the theoretical point of view, an exhaustive analysis and extension of the results presented in this article to systems not in normal form and possibly non-minimum phase, as in $[3,4]$, remains an open problem. 


\begin{tabular}{l|ll|ll} 
& \multicolumn{2}{c}{ no measurement noise } & \multicolumn{2}{c}{ with measurement noise } \\
\hline$\sigma$ & $\sup _{t \in[0, T]}\left|e_{p}(t)\right|$ & $\frac{1}{T} \int_{0}^{T}\left|e_{p}(t)\right|^{2} d t$ & $\sup _{t \in[0, T]}\left|e_{p}(t)\right|$ & $\frac{1}{T} \int_{0}^{T}\left|e_{p}(t)\right|^{2} d t$ \\
\hline 2 & 1.2555 & 0.9657 & 1.3023 & 0.9743 \\
5 & 0.6577 & 0.4083 & 0.7995 & 0.4244 \\
10 & 0.400 & 0.2166 & 0.6619 & 0.2489 \\
20 & 0.2248 & 0.1126 & 0.6201 & 0.1765 \\
40 & 0.1181 & 0.0572 & 0.6736 & 0.1735 \\
\hline
\end{tabular}

Table 1: Simulation of the example (26) with high-gain feedback control (25), for different selections of $\sigma$, with and without measurement noise. The noise is generated as random white noise coloured with a high-pass filter.

\begin{tabular}{|c|c|c|c|c|c|c|c|}
\hline & & & & \multicolumn{2}{|c|}{ no measurement noise } & \multicolumn{2}{|c|}{ with measurement noise } \\
\hline$\sigma$ & $\mu$ & $\widehat{\omega}$ & $n_{o}$ & $\sup _{t \in[0, T]}\left|e_{p}(t)\right|$ & $\frac{1}{T} \int_{0}^{T}\left|e_{p}(t)\right|^{2} d t$ & $\sup _{t \in[0, T]}\left|e_{p}(t)\right|$ & $\frac{1}{T} \int_{0}^{T}\left|e_{p}(t)\right|^{2} d t$ \\
\hline 2 & 1 & - & 0 & 0.3074 & 0.1777 & 0.3915 & 0.2024 \\
\hline 2 & 1 & $2 \pi$ & 1 & 0.0917 & 0.0549 & 0.2453 & 0.0872 \\
\hline 2 & 1 & $2 \pi$ & 2 & 0.0178 & 0.0099 & 0.1822 & 0.0545 \\
\hline 2 & 1 & $2 \pi$ & 3 & 0.0049 & 0.0035 & 0.1679 & 0.0514 \\
\hline 2 & 1 & $2 \pi$ & 4 & $3.04 \cdot 10^{-8}$ & $1.66 \cdot 10^{-8}$ & 0.1730 & 0.0523 \\
\hline 2 & 1 & $0.99 \cdot 2 \pi$ & 1 & 0.1145 & 0.0587 & 0.2716 & 0.0985 \\
\hline 2 & 1 & $0.99 \cdot 2 \pi$ & 2 & 0.0835 & 0.0371 & 0.2391 & 0.0814 \\
\hline 2 & 1 & $0.99 \cdot 2 \pi$ & 3 & 0.0837 & 0.0369 & 0.2292 & 0.0790 \\
\hline 2 & 1 & $0.95 \cdot 2 \pi$ & 1 & 0.2045 & 0.0996 & 0.3693 & 0.1478 \\
\hline 2 & 1 & $0.95 \cdot 2 \pi$ & 2 & 0.2038 & 0.0980 & 0.3818 & 0.1498 \\
\hline 2 & 1 & $0.95 \cdot 2 \pi$ & 3 & 0.2041 & 0.0982 & 0.3876 & 0.1499 \\
\hline 2 & 1 & $2 \pi \varphi_{g}$ & 1 & 0.2915 & 0.1788 & 0.3764 & 0.2020 \\
\hline 2 & 1 & $2 \pi \varphi_{g}$ & 2 & 0.2928 & 0.1790 & 0.3761 & 0.2017 \\
\hline 2 & 1 & $2 \pi \varphi_{g}$ & 3 & 0.2929 & 0.1790 & 0.3792 & 0.2015 \\
\hline
\end{tabular}

Table 2: Simulation of the example (26) with the regulator (9), for different selections of $n_{o}$ and $\hat{\omega}$, with $n_{z \ell}$ selected as in (8) with $\varepsilon=0.5$. Note that $\varphi_{g}=\frac{1+\sqrt{5}}{2}$ corresponds to the golden number. 


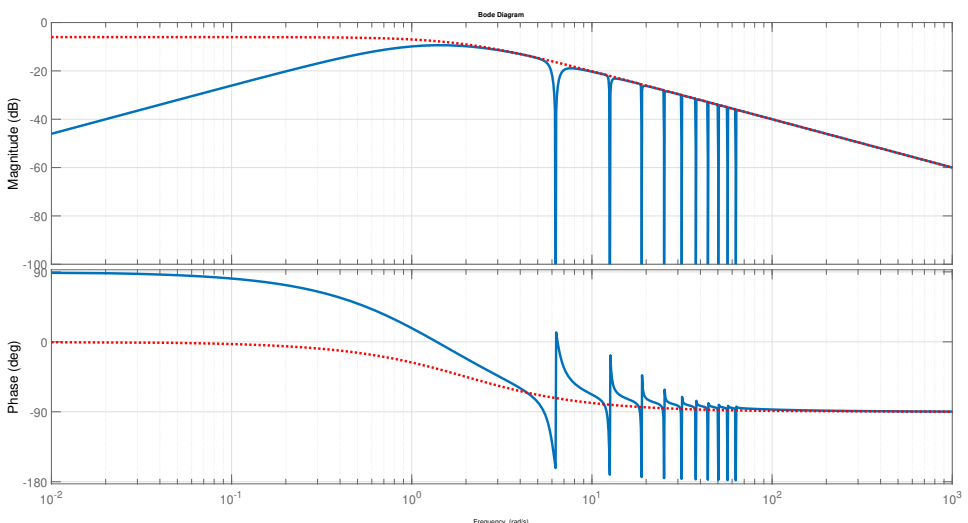

Fig. 1: Bode diagram of the transfer between $e$ and $q$ for the closed-loop systems (24), (25), in blue, respectively (24), (9), in red.

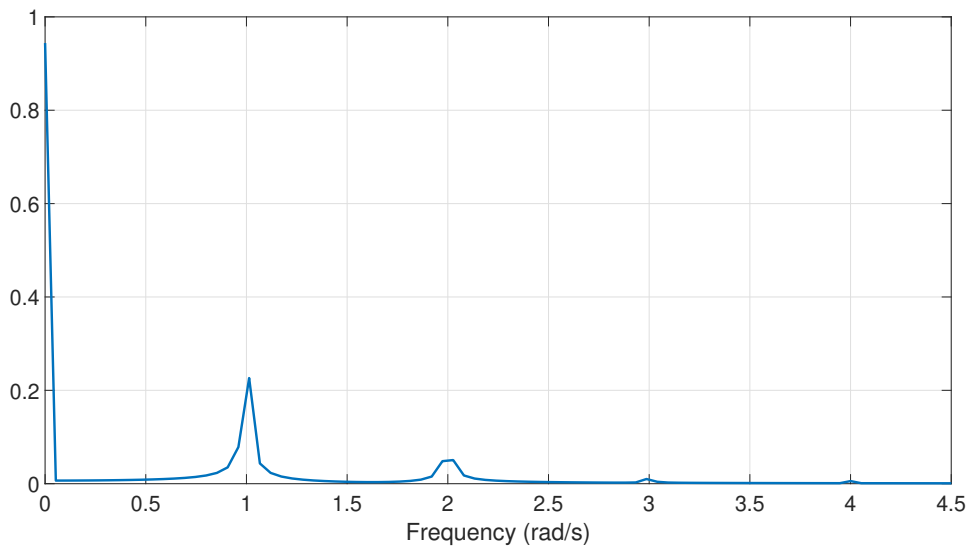

Fig. 2: FFT (fast Fourier transform) of the steady-state regulated error $e_{p}(t)$ for the example (26) with high-gain feedback (25), with $\sigma=2$.

\section{A Proof of Theorem 1}

A simple way to establish Theorem 1 could be by showing that the origin of (28) for $q=0$ is exponentially stable, and then perturb such solution with a small $q$. Fixed point theorems and exponential stability arguments would prove the desired result, see, e.g., Theorem 3.1, Chapter 8.3, in [39]. However, in doing so, all the results would be $n_{o}$-dependent. Since the objective of this proof is to show that this is not the case, namely the existence of a stable periodic solution is verified for any choice of $n_{o}$, with bounds that do not depend on $n_{o}$, we are forced to redo the proof, following the classical route but re-entering into the details and being careful and precise with the bounds. 


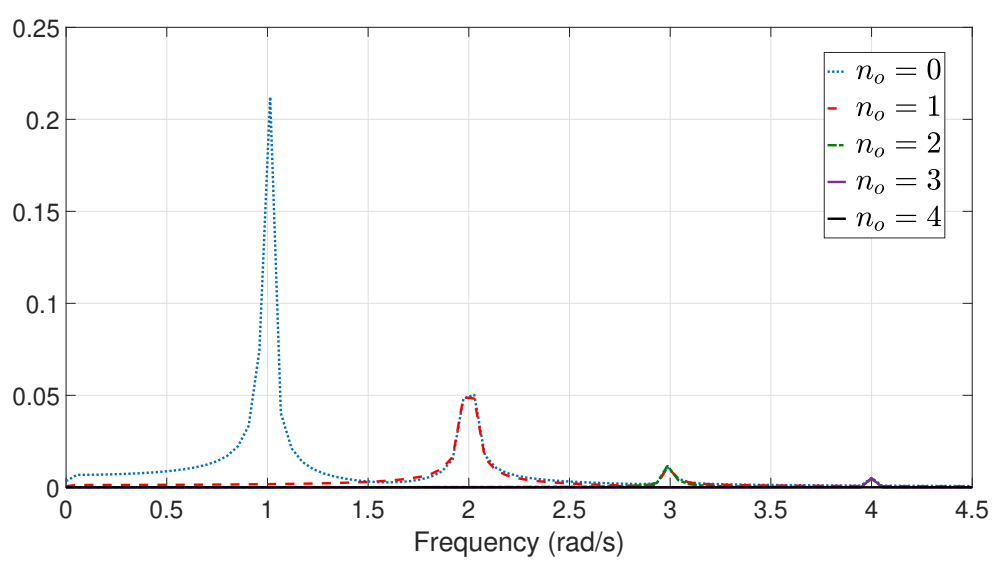

Fig. 3: FFT (fast Fourier transform) of the steady-state regulated error $e_{p}(t)$ for the example (26) with controller (9), and parameters selected as $\sigma=2, \mu=1, \varepsilon=0.5$, $n_{o}=\{0, \ldots, 4\}$ and $\widehat{\omega}=\frac{2 \pi}{T}$.

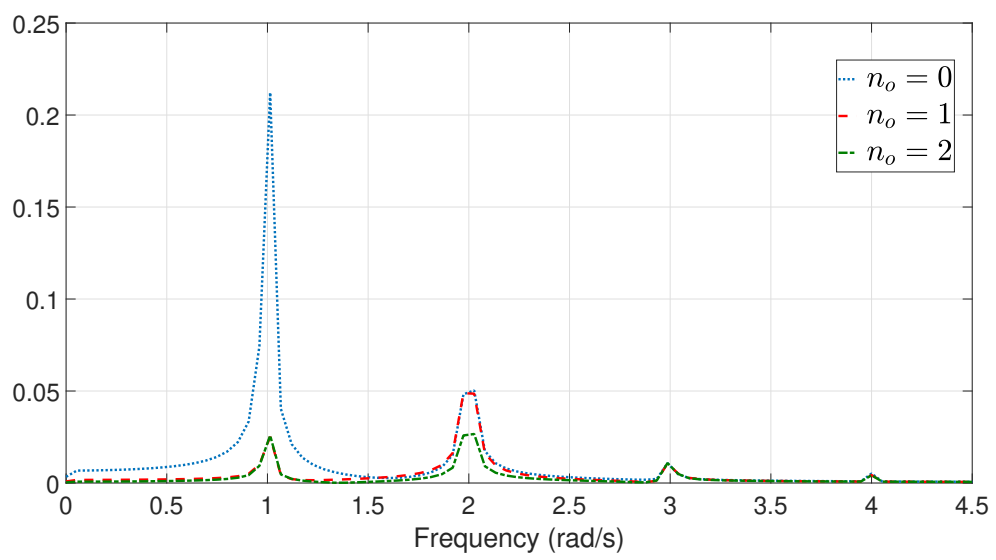

Fig. 4: FFT (fast Fourier transform) of the steady-state regulated error $e_{p}(t)$ for the example (26) with controller (9), and parameters selected as $\sigma=2, \mu=1, \varepsilon=0.5$, $n_{o}=\{0,1,2\}$ and $\widehat{\omega}=0.99 \frac{2 \pi}{T}$. For $n_{o}=3$, the line is nearly overlapped with the one of $n_{o}=2$.

\section{A.1 Preliminaries}

First, let us make the following change of coordinates

$$
z \mapsto \zeta:=z-M e
$$




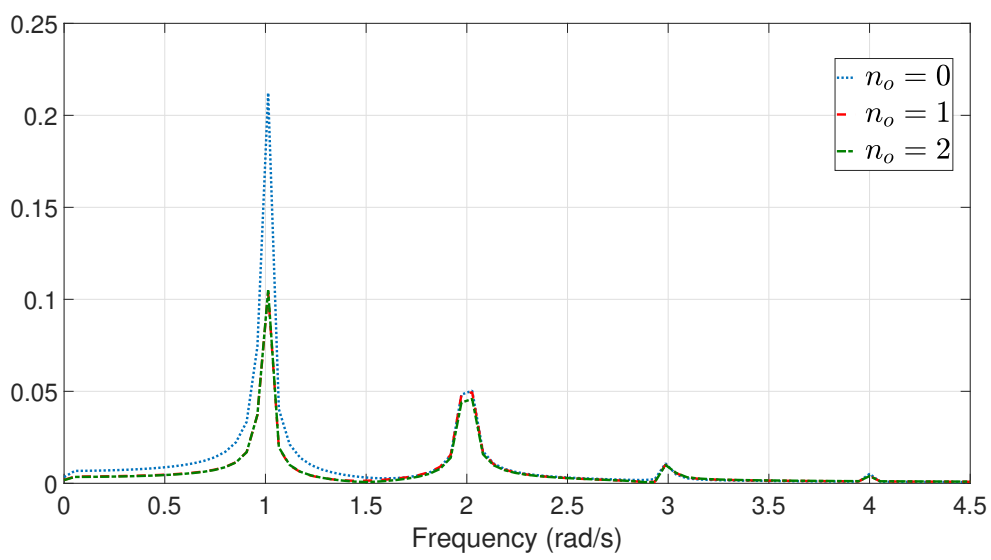

Fig. 5: FFT (fast Fourier transform) of the steady-state regulated error $e_{p}(t)$ for the example (26) with controller (9), and parameters selected as $\sigma=2, \mu=1, \varepsilon=0.5$, $n_{o}=\{0,1,2\}$ and $\widehat{\omega}=0.95 \frac{2 \pi}{T}$. From $n_{o}>1$ the curves are nearly overlapped.

which transform the closed-loop system (1), (9) into

$$
\begin{aligned}
& \dot{x}=f(t, x, e) \\
& \dot{e}=q(t, x, e)-\sigma e+\mu M^{\top} N_{z} \zeta \\
& \dot{\zeta}=\left(\Phi-\mu M M^{\top} N_{z}\right) \zeta-M q(t, x, e) .
\end{aligned}
$$

Our approach to study this system is to decompose it as :

$$
\begin{aligned}
\dot{x}^{+} & =\frac{\partial f}{\partial x}(t, 0,0) x^{+}+\left[f\left(t, x^{-}, e^{-}\right)-\frac{\partial f}{\partial x}(t, 0,0) x^{-}\right] \\
\dot{e}^{+} & =-\sigma e^{+}+\left[q\left(t, x^{-}, e^{-}\right)+\eta\right]
\end{aligned}
$$

where $x^{-}$and $e^{-}$are inputs and $\eta$ is the output of the system

$$
\dot{\zeta}=\left(\Phi-\mu M M^{\top} N_{z}\right) \zeta-M q\left(t, x^{-}, e^{-}\right), \quad \eta=\mu M^{\top} N_{z} \zeta
$$

Indeed we recover the system (28) when inputs $\left(x^{-}, e^{-}\right)$equal outputs $\left(x^{+}, e^{+}\right)$and we can benefit from the following properties :

- The origin of the subsystem $\dot{x}=f(t, x, 0)$ and therefore of $\dot{x}^{+}=\frac{\partial f}{\partial x}(t, 0,0) x^{+}$is locally exponentially stable in light of (4), (5).

- The origin of subsystem $\dot{e}=-\sigma e$ is exponentially stable.

- The (linear) subsystem (30) with input $q$ and output $\eta$ is linear and we shall show in Lemma 1 below it is stable.

We give the $\zeta$ subsystem (30) a special treatment because of its strong dependence on $n_{o}$. Annoying features are, for example,

- the dimension of $\zeta$ is $2 n_{o}+1$;

- $\operatorname{trace}\left(\Phi-\mu M M^{\top} N_{z}\right)=\mu\left[1+\sum_{k=1}^{n_{o}} n_{z k}\right]$

which, with (7a) and Lemma 1, implies the real part of the eigen values of $\left(\Phi-\mu M M^{\top} N_{z}\right)$ tends to 0 as $n_{o}$ tends to infinity. 
In the following, we start by studying the $\zeta$-subsystem. Then we show that, with a suitable choice of $\sigma, \mu$ and bounds $\mathbf{x}, \mathbf{e}$, arguments of our bounding functions in (3) and (6) for $f, \frac{\partial f}{\partial x}$ and $q$, there is a periodic solution $\left(x_{p}, e_{p}\right)$ satisfying :

$$
\left(x_{p}, e_{p}\right)=\left(x^{-}, e^{-}\right)=\left(x^{+}, e^{+}\right)
$$

Finally we prove it is exponentially stable and study its domain of attraction and its properties.

A.2 Study of the system (30)

Lemma 1 Let $\Phi, M, N_{z}$ be defined as in (10). Then, for any $n_{o} \in \mathbb{N}$ and any $\mu>0$, the pair $\left(\Phi, M^{\top} N_{z}\right)$ is observable and the matrix $\left(\Phi-\mu M M^{\top} N_{z}\right)$ is Hurwitz. In particular, there exist a symmetric positive definite matrix $P_{\zeta}$, depending on $n_{o}$, and, for any strictly positive real number $\mu$, there exists a strictly positive real number $\kappa$, depending on $n_{o}$, such that we have

$$
\left(N_{z}+\kappa P_{\zeta}\right)\left(\Phi-\mu M M^{\top} N_{z}\right)+\left(\Phi-\mu M M^{\top} N_{z}\right)^{\top}\left(N_{z}+\kappa P_{\zeta}\right) \leq-\mu N_{z} M M^{\top} N_{z}-\kappa N_{z} .
$$

Proof The pair $\left(\Phi_{\ell}, M_{\ell}\right)$ in (10) is observable. Then, observability of $\left(\Phi, M^{\top} N_{z}\right)$ is a direct consequence of the block-diagonal structure of the matrix $\Phi$ and the fact that $N_{z}$ is diagonal. Since the pair $\left(\Phi, M^{\top} N_{z}\right)$ is observable and $N_{z}$ is positive definite, there exist a matrix $K$ and a positive definite matrix $P_{\zeta}$ satisfying

$$
P_{\zeta}\left(\Phi-K M^{\top} N_{z}\right)+\left(\Phi-K M^{\top} N_{z}\right)^{\top} P_{\zeta}=-2 N_{z}
$$

On another hand, since (10) implies $N_{z} \Phi+\Phi^{\top} N_{z}=0$, we obtain, adding and subtracting the term $\mu N_{z} M M^{\top} N_{z}$,

$$
N_{z}\left(\Phi-\mu M M^{\top} N_{z}\right)+\left(\Phi-\mu M M^{\top} N_{z}\right)^{\top} N_{z}+2 \mu N_{z} M M^{\top} N_{z}=0 .
$$

By combining these two equations, we get, with $\kappa$ any strictly positive real number,

$$
\begin{aligned}
\left(N_{z}+\kappa P_{\zeta}\right)\left(\Phi-\mu M M^{\top} N_{z}\right)+\left(\Phi-\mu M M^{\top} N_{z}\right)^{\top}\left(N_{z}+\kappa P_{\zeta}\right) & \\
=-2 \mu N_{z} M M^{\top} N_{z}-2 \kappa N_{z}-\kappa \mu\left(P_{\zeta} M M^{\top} N_{z}\right. & \left.+N_{z} M M^{\top} P_{\zeta}\right) \\
& +\kappa\left(P_{\zeta} K M^{\top} N_{z}+N_{z} M K^{\top} P_{\zeta}\right)
\end{aligned}
$$

But, for any matrices $A$ and $B$ and real numbers $\kappa$ and $\mu$, we have the following identity

$$
\mu A A^{\top}+\kappa\left(B A^{\top}+A B^{\top}\right)=\left(\sqrt{\mu} A+\frac{\kappa}{\sqrt{\mu}} B\right)\left(\sqrt{\mu} A+\frac{\kappa}{\sqrt{\mu}} B\right)^{\top}-\frac{\kappa^{2}}{\mu} B B^{\top} .
$$

By using previous identity in which $A=N_{z} M$ and $B=P_{\zeta}(\mu M-K)$, we obtain

$$
\begin{array}{r}
-\mu N_{z} M M^{\top} N_{z}-\kappa \mu\left(P_{\zeta} M M^{\top} N_{z}+N_{z} M M^{\top} P_{\zeta}\right)+\kappa\left[P_{\zeta} K M^{\top} N_{z}+N_{z} M K^{\top} P_{\zeta}\right] \\
=-\left(\sqrt{\mu} N_{z} M+\frac{\kappa}{\sqrt{\mu}} P_{\zeta}(\mu M-K)\right)\left(\sqrt{\mu} N_{z} M+\frac{\kappa}{\sqrt{\mu}} P_{\zeta}(\mu M-K)\right)^{\top} \\
+\frac{\kappa^{2}}{\mu} P_{\zeta}(\mu M-K)(\mu M-K)^{\top} P_{\zeta} .
\end{array}
$$

Hence, by combining such identity with (32), we obtain

$$
\begin{aligned}
\left(N_{z}+\kappa P_{\zeta}\right)\left(\Phi-\mu M M^{\top} N_{z}\right)+\left(\Phi-\mu M M^{\top} N_{z}\right)^{\top}\left(N_{z}+\kappa P_{\zeta}\right) & \\
=-\left(\sqrt{\mu} N_{z} M+\right. & \left.\frac{\kappa}{\sqrt{\mu}} P_{\zeta}(\mu M-K)\right)\left(\sqrt{\mu} N_{z} M+\frac{\kappa}{\sqrt{\mu}} P_{\zeta}(\mu M-K)\right)^{\top} \\
& \quad-\kappa\left(N_{z}-\frac{\kappa}{\mu} P_{\zeta}(\mu M-K)(\mu M-K)^{\top} P_{\zeta}\right)-\mu N_{z} M M^{\top} N_{z}-\kappa N_{z} .
\end{aligned}
$$


Finally, by selecting $\kappa$ small enough so that

$$
N_{z} \geq \frac{\kappa}{\mu} P_{\zeta}(\mu M-K)(\mu M-K)^{\top} P_{\zeta},
$$

we obtain (31)

Lemma 2 Consider system

$$
\dot{\zeta}=\left(\Phi-\mu M M^{\top} N_{z}\right) \zeta-M v
$$

with $\Phi, M, N_{z}$ be defined as in (10) and $n_{z k}$ satisfying (7a), e.g. as in (8). The transfer function between $v$ and $\mathscr{N}_{z}^{\frac{1}{2}} \zeta$ satisfies

$$
\bar{\zeta}(\mathrm{i} \omega)^{\top} N_{z} \zeta(\mathrm{i} \omega)=\frac{\sum_{\ell=0}^{n_{o}} \frac{\omega^{2}+\omega_{\ell}^{2}}{\left(\omega_{\ell}^{2}-\omega^{2}\right)^{2}} n_{z \ell}}{1+\mu^{2}\left(\sum_{\ell=0}^{n_{o}} \frac{\omega}{\omega_{\ell}^{2}-\omega^{2}} n_{z \ell}\right)^{2}}|v(\mathrm{i} \omega)|^{2} .
$$

Furthermore, there exists $\kappa_{0}, \kappa_{1}>0$ independent of $n_{o}$ such that

$$
\bar{\zeta}(\mathrm{i} \omega)^{\top} N_{z} \zeta(\mathrm{i} \omega) \leq\left(\kappa_{0}+\kappa_{1} \omega^{2}\right)|v(\mathrm{i} \omega)|^{2} \quad \forall \omega \in \mathbb{R} .
$$

Proof Consider the change of coordinates $\eta \mapsto y:=N_{z}^{\frac{1}{2}} \zeta$ giving

$$
\dot{y}=\left(\Phi-\mu N_{z}^{\frac{1}{2}} M M^{\top} N_{z}^{\frac{1}{2}}\right) y-N_{z}^{\frac{1}{2}} M v .
$$

By defining with $G(\mathrm{i} \omega)$ the transfer function between $v$ and $y$, it is readily seen that it can be computed as

$$
G(\mathrm{i} \omega)=\left[\mathrm{i} \omega I-\Phi+\mu N_{z}^{\frac{1}{2}} M M^{\top} N_{z}^{\frac{1}{2}}\right]^{-1} N_{z}^{\frac{1}{2}} M
$$

and its transpose conjugate given by

$$
G^{*}(\mathrm{i} \omega)=M^{\top} N_{z}^{\frac{1}{2}}\left[-\mathrm{i} \omega I+\Phi+\mu N_{z}^{\frac{1}{2}} M M^{\top} N_{z}^{\frac{1}{2}}\right]^{-1}
$$

where we used the fact that $\Phi^{\top}=-\Phi$. By temporarily using the compact notation $\Lambda:=\mathrm{i} \omega I-\Phi$, and $\Upsilon:=\mu^{\frac{1}{2}} N_{z}^{\frac{1}{2}} M$, and by Woodbury matrix identity ${ }^{4}$ (recall that $\Upsilon$ is a vector), we obtain

$$
\begin{aligned}
G^{*}(\mathrm{i} \omega) G(\mathrm{i} \omega) & =\frac{1}{\mu} \Upsilon^{\top}\left(-\Lambda+\Upsilon \Upsilon^{\top}\right)^{-1}\left(\Lambda+\Upsilon \Upsilon^{\top}\right)^{-1} \Upsilon \\
& =\frac{1}{\mu} \Upsilon^{\top}\left[(-\Lambda)^{-1}-\frac{\Lambda^{-1} \Upsilon \Upsilon^{\top} \Lambda^{-1}}{1+\Upsilon^{\top}\left(-\Lambda^{-1}\right)}\right]\left[\Lambda^{-1}-\frac{\Lambda^{-1} \Upsilon \Upsilon^{\top} \Lambda^{-1}}{1+\Upsilon^{\top} \Lambda^{-1} \Upsilon}\right] \Upsilon \\
& =\frac{1}{\mu} \frac{-\Upsilon^{\top} \Lambda}{1-\Upsilon^{\top} \Lambda^{-1} \Upsilon} \frac{\Lambda \Upsilon}{1+\Upsilon^{\top} \Lambda^{-1} \Upsilon}=-\frac{1}{\mu} \frac{\Upsilon^{\top} \Lambda^{-2} \Upsilon}{1-\left(\Upsilon^{\top} \Lambda^{-1} \Upsilon\right)^{2}} \\
& =\frac{-M^{\top} N_{z}^{\frac{1}{2}}(\mathrm{i} \omega I-\Phi)^{-2} N_{z}^{\frac{1}{2}} M}{1-\mu^{2}\left(M^{\top} N_{z}^{\frac{1}{2}}(i \omega I-\Phi)^{-1} N_{z}^{\frac{1}{2}} M\right)^{2}} .
\end{aligned}
$$

Now, note that we have algebraically

$$
\begin{aligned}
& \left(\mathrm{i} \omega I-\Phi_{\ell}\right)^{-1}=\left(\begin{array}{cc}
\mathrm{i} \omega & -\omega_{\ell} \\
\omega_{\ell} & \mathrm{i} \omega
\end{array}\right)^{-1}=\frac{1}{\omega_{\ell}^{2}-\omega^{2}}\left(\begin{array}{cc}
\mathrm{i} \omega & \omega_{\ell} \\
-\omega_{\ell} & \mathrm{i} \omega
\end{array}\right) \\
& M_{\ell}^{\top} N_{z \ell}^{\frac{1}{2}}\left(\mathrm{i} \omega I-\Phi_{\ell}\right)^{-1} N_{z \ell}^{\frac{1}{2}} M_{\ell}=\frac{\mathrm{i} \omega}{\omega_{\ell}^{2}-\omega^{2}}\left|M_{\ell}\right|^{2} n_{z \ell} .
\end{aligned}
$$

4 Also known as matrix inversion lemma, the Woodbury matrix identity states that for $A$ invertible and $u, v$ column vectors, the following holds: $\left(A+u v^{\top}\right)^{-1}=A^{-1}-\frac{1}{1+v^{\top} A^{-1} u} A^{-1} u v^{\top} A^{-1}$. 
Moreover,

$$
\begin{aligned}
& \left(\mathrm{i} \omega I-\Phi_{\ell}\right)^{-2}=\left(\begin{array}{cc}
\mathrm{i} \omega & -\omega_{\ell} \\
\omega_{\ell} & \mathrm{i} \omega
\end{array}\right)^{-2}=\frac{-1}{\left(\omega_{\ell}^{2}-\omega^{2}\right)^{2}}\left(\begin{array}{cc}
\omega^{2}+\omega_{\ell}^{2} & -2 \mathrm{i} \omega \omega_{\ell} \\
2 \mathrm{i} \omega \omega_{\ell} & \omega^{2}+\omega_{\ell}^{2}
\end{array}\right) \\
& M_{\ell}^{\top} N_{z \ell}^{\frac{1}{2}}\left(\mathrm{i} \omega I-\Phi_{\ell}\right)^{-2} N_{z \ell}^{\frac{1}{2}} M_{\ell}=-\frac{\omega^{2}+\omega_{\ell}^{2}}{\left(\omega_{\ell}^{2}-\omega^{2}\right)^{2}}\left|M_{\ell}\right|^{2} n_{z \ell} .
\end{aligned}
$$

This yields

$$
\begin{gathered}
M_{\ell}^{\top} N_{z \ell}^{\frac{1}{2}}\left(\mathrm{i} \omega I-\Phi_{\ell}\right)^{-1} N_{z \ell}^{\frac{1}{2}} M_{\ell}=\mathrm{i} \sum_{\ell=0}^{n_{o}} \frac{\omega}{\omega_{\ell}^{2}-\omega^{2}}\left|M_{\ell}\right|^{2} n_{z \ell} \\
M_{\ell}^{\top} N_{z \ell}^{\frac{1}{2}}\left(\mathrm{i} \omega I-\Phi_{\ell}\right)^{-2} N_{z \ell}^{\frac{1}{2}} M_{\ell}=-\sum_{\ell=0}^{n_{o}} \frac{\omega^{2}+\omega_{\ell}^{2}}{\left(\omega_{\ell}^{2}-\omega^{2}\right)^{2}}\left|M_{\ell}\right|^{2} n_{z \ell}
\end{gathered}
$$

which finally gives the expression (34) in which we used also the definition $M_{\ell}=(1,0)$ and the block diagonal form of the matrix $\Phi$.

We are left with proving inequality (35). By letting $x=\frac{\omega}{\omega}$, and recalling (7e), the fraction in (34) reads

$$
\mathcal{T}(x)=\frac{\sum_{\ell=0}^{n_{o}} \frac{\omega^{2}+\omega_{\ell}^{2}}{\left(\omega_{\ell}^{2}-\omega^{2}\right)^{2}} n_{z \ell}}{1+\mu^{2}\left(\sum_{\ell=0}^{n_{o}} \frac{\omega}{\omega_{\ell}^{2}-\omega^{2}} n_{z \ell}\right)^{2}}=\frac{\sum_{\ell=0}^{n_{o}} \frac{\ell^{2}+x^{2}}{\left(\ell^{2}-x^{2}\right)^{2}} n_{z \ell}}{1+\mu^{2} x^{2}\left(\sum_{\ell=0}^{n_{o}} \frac{1}{\ell^{2}-x^{2}} n_{z \ell}\right)^{2}} .
$$

The rest of the proof follows by direct application of Lemma 6 given in the Supplementary Material at the end of this article.

Remark 1 The expression (34) can be also rewritten as

$$
\frac{\bar{\zeta}(\mathrm{i} \omega)^{\top} N_{z} \zeta(\mathrm{i} \omega)}{|v(\mathrm{i} \omega)|^{2}}=\frac{\sum_{\ell=0}^{n_{o}}\left(\omega_{\ell}^{2}+\omega^{2}\right) n_{z \ell}\left[\prod_{m=0, \neq \ell}^{n_{o}}\left(\omega_{m}^{2}-\omega^{2}\right)\right]^{2}}{\left[\prod_{m=0}^{n_{o}}\left(\omega_{m}^{2}-\omega^{2}\right)\right]^{2}+\mu^{2} \omega^{2}\left[\sum_{\ell=0}^{n_{o}} n_{z \ell} \prod_{m=0, \neq \ell}^{n_{o}}\left(\omega_{m}^{2}-\omega^{2}\right)\right]^{2}}
$$

showing that there is actually no singularity at $\omega=\omega_{\ell}$, and we have in particular

$$
\frac{\bar{\zeta}\left(\mathrm{i} \omega_{\ell}\right)^{\top} N_{z} \zeta\left(\mathrm{i} \omega_{\ell}\right)}{\left|v\left(\mathrm{i} \omega_{\ell}\right)\right|^{2}}=\frac{2}{\mu^{2} n_{z \ell}} .
$$

Lemma 3 Consider again system

$$
\dot{\zeta}=\left(\Phi-\mu M M^{\top} N_{z}\right) \zeta-M v(t)
$$

with $v \in \mathscr{C}_{T}^{1}(\mathbb{R})$ and $\Phi, M, N_{z}$ be defined as in (10) and $n_{z k}$ satisfying (7a), e.g. as in (8). For any $n_{o} \in \mathbb{N}$, it has a unique periodic solution $\zeta_{p}$ satisfying

$$
\begin{gathered}
\mu^{2} \int_{0}^{T}\left|M^{\top} N_{z} \zeta_{p}(t)\right|^{2} d t \leq \int_{0}^{T}|v(t)|^{2} d t \\
\sup _{t \in[0, T]} \zeta_{p}(t)^{\top} N_{z} \zeta_{p}(t) \leq\left(\frac{1}{\mu}+\frac{\kappa_{0}}{T}\right) \int_{0}^{T}|v(t)|^{2} d t+\frac{\kappa_{1}}{T} \int_{0}^{T}|\dot{v}(t)|^{2} d t
\end{gathered}
$$

with $\kappa_{0}, \kappa_{1}$ given by Lemma 2 .

Proof According to Lemma 1, the matrix $\Phi-\mu M M^{\top} N_{z}$ is Hurwitz. Hence (see Lemma 4) system (38) admits a unique periodic solution given by

$$
\zeta_{p}(t)=\Psi\left(\Phi-\mu M M^{\top} N_{z},-M v(t)\right)
$$


with $\Psi$ defined below in (52). Then, compute

$$
\begin{aligned}
\frac{1}{2} \overparen{\zeta_{p}(t)^{\top} N_{z} \zeta_{p}(t)} & =\zeta_{p}(t)^{\top} N_{z}\left[\Phi-\mu M M^{\top} N_{z}\right] \zeta_{p}(t)-\zeta_{p}(t)^{\top} N_{z} M v(t) \\
& =-\mu\left|M^{\top} N_{z} \zeta_{p}(t)\right|^{2}-\zeta_{p}(t)^{\top} N_{z} M v(t) \\
& \leq-\frac{\mu}{2}\left|M^{\top} N_{z} \zeta_{p}(t)\right|^{2}+\frac{1}{2 \mu}|v(t)|^{2} .
\end{aligned}
$$

By integrating and using periodicity, this yields to (39).

In order to show the second inequality of the statement of the lemma, the function $v$ being in $\mathscr{C}_{T}^{1}(\mathbb{R})$ can be expressed as the sum of its Fourier series as

$$
v(t)=\sum_{k \in \mathbb{Z}} v_{k} \exp \left(\mathrm{i} k \frac{2 \pi}{T} t\right)
$$

By using the Bessel-Parseval identity and continuity of the derivative $\dot{v}$, we have

$$
\left[\frac{2 \pi}{T}\right]^{2} \sum_{k=0}^{\infty} k^{2}\left|v_{k}\right|^{2} \leq \frac{1}{T} \int_{0}^{T}|\dot{v}(t)|^{2} d t
$$

We have similarly

$$
\frac{1}{T} \int_{0}^{T} \zeta_{p}(t)^{\top} N_{z} \zeta_{p}(t) d t=\sum_{k=0}^{\infty} \bar{\zeta}_{p k}^{\top} N_{z} \zeta_{p k}, \quad \zeta_{p k}=\frac{1}{T} \int_{0}^{T} \zeta_{p}(t) \exp \left(\mathrm{i} k \frac{2 \pi}{T} t\right) d t
$$

Now, by applying the expression of the transfer function (34) to each element of the Fourier series of the product $\zeta_{p}(t)^{\top} N_{z} \zeta_{p}(t)$, we obtain

$$
\bar{\zeta}_{p k}^{\top} N_{z} \zeta_{p k}=\frac{\sum_{\ell=0}^{n_{o}} \frac{\omega_{\ell}^{2}+\left[\frac{2 \pi}{T}\right]^{2} k^{2}}{\left(\omega_{\ell}^{2}-\left[\frac{2 \pi}{T}\right]^{2} k^{2}\right)^{2}} n_{z \ell}}{1+\mu^{2}\left(\sum_{\ell=0}^{n_{o}} \frac{\left[\frac{2 \pi}{T}\right]^{2} k^{2}}{\omega_{\ell}^{2}-\left[\frac{2 \pi}{T}\right]^{2} k^{2}} n_{z \ell}\right)^{2}}\left|v_{k}\right|^{2} .
$$

As a consequence, by using inequality (35) on previous identity, and by using again equations (42) and (43), we further obtain

$$
\begin{aligned}
\sum_{k=0}^{\infty} \bar{\zeta}_{p k}^{\top} N_{z} \zeta_{p k} & \leq \sum_{k=0}^{\infty}\left(\kappa_{0}+\kappa_{1}\left[\frac{2 \pi}{T}\right]^{2} k^{2}\right)\left|v_{k}\right|^{2} \\
& \leq \frac{\kappa_{0}}{T} \int_{0}^{T}|v(s)|^{2} d s+\frac{\kappa_{1}}{T} \int_{0}^{T}|\dot{v}(s)|^{2} d s .
\end{aligned}
$$

We note also that, as a consequence of (41), we have

$$
\sup _{t \in[0, T]} \zeta_{p}(t)^{\top} N_{z} \zeta_{p}(t)-\inf _{t \in[0, T]} \zeta_{p}(t)^{\top} N_{z} \zeta_{p}(t) \leq \frac{1}{\mu} \int_{0}^{T}|v(s)|^{2} d s
$$

On another hand, we have

$$
\inf _{t \in[0, T]} \zeta_{p}(t)^{\top} N_{z} \zeta_{p}(t) \leq \frac{1}{T} \int_{0}^{T} \zeta_{p}(t)^{\top} N_{z} \zeta_{p}(t) d s .
$$

This yields

$$
\sup _{t \in[0, T]} \zeta_{p}(t)^{\top} N_{z} \zeta_{p}(t) \leq \frac{1}{T} \int_{0}^{T} \zeta_{p}(t)^{\top} N_{z} \zeta_{p}(t) d s+\frac{1}{\mu} \int_{0}^{T}|v(s)|^{2} d s,
$$

Finally, by combining (45) with (43) and (44), we obtain (40). 


\section{A.3 Existence of a Periodic Solution}

Proposition 1 For any triplet $\left(\bar{P}_{x}, \underline{P}_{x}, \alpha\right)$, there exist strictly positive real numbers $\mathbf{x}_{p}, \mathbf{e}_{p}, \boldsymbol{\zeta}_{p}, \mathbf{r}_{x}, \mathbf{r}_{e}, \sigma_{p}^{\star}>0$ (independent of $n_{o}$ ) such that, for any $n_{o}>0$, any $\sigma>\sigma_{p}^{\star}$, and $\mu \geq 1$, system $(28)$, with $f \in \mathscr{F}\left(\mathbf{x}_{p}, \mathbf{e}_{p}, \bar{P}_{x}, \underline{P}_{x}, \alpha\right)$ and $q \in \mathscr{Q}\left(\mathbf{x}_{p}, \mathbf{e}_{p}\right)$, admits a T-periodic solution $\left(x_{p}, e_{p}, \zeta_{p}\right) \in \mathscr{C}_{T}^{2}\left([0, T] ; \mathbb{R}^{n} \times \mathbb{R} \times \mathbb{R}^{2 n_{o}+1}\right)$ satisfying, for all $t \in[0, T]$, the following inequalities

$$
\begin{aligned}
\left|x_{p}(t)\right| & \leq \min \left\{\mathbf{x}_{p}, \frac{\mathbf{r}_{x}}{\sigma}\right\}, \\
\left|e_{p}(t)\right| & \leq \min \left\{\mathbf{e}_{p}, \frac{\mathbf{r}_{e}}{\sigma}\right\}, \\
\sqrt{\zeta_{p}(t)^{\top} N_{z} \zeta_{p}(t)} & \leq \boldsymbol{\zeta}_{p} .
\end{aligned}
$$

Proof Here we exploit the ability of expressing the system (28) as in (29) and (30). With the notations

$$
\delta f(t, x, e):=f(t, x, e)-F(t) x, \quad F(t):=\frac{\partial f}{\partial x}(t, 0,0),
$$

system (28) reads as follows when $\left(x^{+}, e^{+}\right)=\left(x^{-}, e^{-}\right)$

$$
\begin{aligned}
& \dot{x}^{+}=F(t) x^{+}+\delta f\left(t, x^{-}, e^{-}\right) \\
& \dot{e}^{+}=-\sigma e^{+}+\left[q\left(t, x^{-}, e^{-}\right)+\eta\right],
\end{aligned}
$$

where

$$
\dot{\zeta}=\left(\Phi-\mu M M^{\top} N_{z}\right) \zeta-M q\left(t, x^{-}, e^{-}\right) \quad, \quad \eta=\mu M^{\top} N_{z} \zeta
$$

Written this way, we see that we have a mapping from the input functions $\left(x^{-}, e^{-}\right)$to the functions $\left(x^{+}, e^{+}\right)$, solution of (48), (49), with the intermediate function $\zeta$ solution of (50).

Each subsystem above can be compactly written as :

$$
\dot{\chi}=F(t) \chi+g(t)
$$

where $F$ is $T$-periodic. We have the following very standard result. See, e.g., [20, Lemma 5.1] or [39, Chapter 8.2]

Lemma 4 Consider system (51) with $g$ is in $\mathscr{C}_{T}^{0}\left(\mathbb{R}^{n}\right)$. If the matrix $\left[I-\phi_{F}(t, t-T)\right]$ is invertible with $\phi_{F}(t, s)$ denoting the state transition matrix of $F$, system (51) admits a unique periodic solution $\chi_{p}$ which can be expressed as

$$
\chi_{p}(t)=\Psi(F(t), g(t)):=\left[I-\phi_{F}(t, t-T)\right]^{-1} \int_{t-T}^{t} \phi_{F}(t, s) g(s) d s .
$$

Furthermore, if there exists a T-periodic function $P_{\chi}$ satisfying

$$
\begin{array}{r}
0<\underline{P}_{\chi} I \leq P_{\chi}(t) \leq \bar{P}_{\chi} I, \\
\dot{P}_{\chi}(t)+P_{\chi}(t) F(t)+F^{\top}(t) P_{\chi}(t) \leq-2 \alpha P_{\chi},
\end{array}
$$

then we have

$$
\begin{aligned}
& \sup _{t \in[0, T]}|\Psi(F(t), g(t))| \leq \frac{K_{\alpha}}{\alpha} \sup _{t \in[0, T]}|g(t)| \\
& \sup _{t \in[0, T]}|\Psi(F(t), g(t))| \leq \frac{K_{\alpha, 2}}{\sqrt{\alpha}} \sup _{t \in[0, T]} \sqrt{\int_{0}^{T} g(t)^{2} d t}
\end{aligned}
$$

where

$$
\begin{aligned}
K_{\alpha} & =\sqrt{\frac{\bar{P}_{\chi}}{\underline{P}_{\chi}}}\left(\exp (\alpha T)-1+\sqrt{\frac{\bar{P}_{\chi}}{\underline{P}_{\chi}}}\right) \exp (-\alpha T), \\
K_{\alpha, 2} & =K_{\alpha} \sqrt{\frac{T(1+\exp (-\alpha T))}{2(1-\exp (-\alpha T))}} .
\end{aligned}
$$


Being interested in periodic solutions for (48), (49) and (50), this leads to the consideration of the following operators

$$
\begin{aligned}
& \mathfrak{O}\left[x^{-}, e^{-}\right]_{x}(t)=\Psi\left(F(t), \delta f\left(t, x^{-}(t), e^{-}(t)\right)\right), \\
& \mathfrak{O}\left[x^{-}, e^{-}\right]_{e}(t)=\Psi\left(-\sigma, q\left(t, x^{-}(t), e^{-}(t)\right)+\eta\left[x^{-}, e^{-}\right](t)\right),
\end{aligned}
$$

where :

$$
\eta\left[x^{-}, e^{-}\right](t)=\mu M^{\top} N_{z} \Psi\left(\Phi-\mu M M^{\top} N_{z},-M q\left(t, x^{-}(t), e^{-}(t)\right) .\right.
$$

When $\left(x^{-}, e^{-}\right)$is $T$-periodic, $\left(\mathfrak{O}\left[x^{-}, e^{-}\right]_{x}, \mathfrak{O}\left[x^{-}, e^{-}\right]_{e}\right)$ is the unique $T$-periodic solution of (48), (49). So to establish our result it is sufficient to show that there exists a $T$-periodic function $\left(x^{-}, e^{-}\right)$satisfying

$$
\left(\mathfrak{O}\left[x^{-}, e^{-}\right]_{x}, \mathfrak{O}\left[x^{-}, e^{-}\right]_{e}\right)=\left(x^{-}, e^{-}\right) .
$$

Our next step is, omitting the superscript ${ }^{-}$to lighten the notations, to show that the operator $(x, e) \mapsto$ $\left(\mathfrak{O}[x, e]_{x}, \mathfrak{O}[x, e]_{e}\right)$ is a contraction on the set of $T$-periodic functions satisfying

$$
\sup _{t \in[0, T]}|x(t)| \leq \mathbf{x}_{p}, \quad \sup _{t \in[0, T]}|e(t)| \leq \mathbf{e}_{p},
$$

when the bounds $\mathbf{x}_{p}, \mathbf{e}_{p}$ are chosen small enough, and $\sigma$ is chosen large enough, this independently of $n_{o}$.

To this end, by recalling the definitions given in Section 1, we list inequalities for the functions $q$ and $f$, obtained as consequences of the following fact

For any $C^{1}$ function $\varphi: \mathbb{R} \times \mathbb{R}^{n} \times \mathbb{R} \rightarrow \mathbb{R}^{m}$ we have

$$
\begin{aligned}
\left|\varphi\left(t, x_{a}, e\right)-\varphi\left(t, x_{b}, e\right)\right| & \left.=\mid\left(\int_{0}^{1} \frac{\partial \varphi}{\partial x}\left(t, x_{b}+s\left(x_{a}-x_{b}\right), e\right)\right) d s\right)\left(x_{a}-x_{b}\right) \mid \\
& \leq \sup _{(t, x, e) \in \mathscr{S}_{T}(\mathbf{x}, \mathbf{e})}\left|\frac{\partial \varphi}{\partial x}(t, x, e)\right|\left|x_{a}-x_{b}\right|
\end{aligned}
$$

for all $x_{a}, x_{b} \in \mathscr{S}_{x}(\mathbf{x}), e \in \mathscr{S}_{e}(\mathbf{e})$, and all $t \in[0, T]$.

First, we have

$$
\begin{aligned}
|q(t, x, e)| & \leq \mathbf{q}(\mathbf{x}, \mathbf{e}) \\
\left|q\left(t, x_{a}, e_{a}\right)-q\left(t, x_{b}, e_{b}\right)\right| & \leq \mathbf{q}_{e}(\mathbf{x}, \mathbf{e})\left|e_{a}-e_{b}\right|+\mathbf{q}_{x}(\mathbf{x}, \mathbf{e})\left|x_{a}-x_{b}\right| \\
\left|f\left(t, x, e_{a}\right)-f\left(t, x, e_{b}\right)\right| & \leq \mathbf{f}_{e}(\mathbf{x}, \mathbf{e})\left|e_{a}-e_{b}\right|
\end{aligned}
$$

for all $x \in \mathscr{S}_{x}(\mathbf{x}), e \in \mathscr{S}_{e}(\mathbf{e}),\left(x_{a}, e_{a}\right)$ and $\left(x_{b}, e_{b}\right)$ in $\mathscr{S}(\mathbf{x}, \mathbf{e})$, and all $t \in[0, T]$. Furthermore, by using the definitions of $F$ and $\delta f$ given in (47), we obtain

$$
\begin{aligned}
|f(t, x, 0)-F(t) x| & \left.=\mid \int_{0}^{1}\left[\left(\frac{\partial f}{\partial x}(t, s x, 0)-\frac{\partial f}{\partial x}(t, 0,0)\right)\right) d s\right] x \mid \\
& =\left|\int_{0}^{1}\left[\int_{0}^{s}\left[\frac{\partial^{2} f}{\partial x \partial x}(t, r x, 0) d r\right] x d s\right] x\right| \leq \frac{1}{2} \mathbf{f}_{x x}(\mathbf{x}, 0)|x|^{2} .
\end{aligned}
$$

By combining the previous bound with (66) in which $e_{a}=e, e_{b}=0$, we get for $\delta f$ defined in (47),

$$
|\delta f(t, x, e)| \leq \mathbf{f}_{e}(\mathbf{x}, \mathbf{e})|e|+\frac{1}{2} \mathbf{f}_{x x}(\mathbf{x}, 0)|x|^{2}
$$

for all $(x, e) \in \mathscr{S}(\mathbf{x}, \mathbf{e})$ and all $t \in[0, T]$. With similar computations, we also obtain

$$
\begin{aligned}
\left|\delta f\left(t, x_{a}, e_{a}\right)-\delta f\left(t, x_{b}, e_{b}\right)\right| \leq \mathbf{f}_{e}(\mathbf{x}, \mathbf{e})\left|e_{a}-e_{b}\right|+\frac{1}{2} \mathbf{f}_{x x}(\mathbf{x}, 0)\left|x_{a}-x_{b}\right|^{2} & \\
+ & +\left[\mathbf{f}_{e x}(\mathbf{x}, \mathbf{e}) \mathbf{e}+\mathbf{f}_{x x}(\mathbf{x}, 0) \mathbf{x}\right]\left|x_{a}-x_{b}\right|
\end{aligned}
$$

for all $\left(x_{a}, e_{a}\right),\left(x_{b}, e_{b}\right) \in \mathscr{S}(\mathbf{x}, \mathbf{e})$ and all $t \in[0, T]$. 
Now, by using the definition of $\mathfrak{O}[x, e]_{x}$ in (59), and the assumptions (4) and (5), inequality (55) gives

$$
\begin{aligned}
\sup _{t \in[0, T]}\left|\mathfrak{O}[x, e]_{x}(t)\right| & \leq \frac{K_{\alpha}}{\alpha} \sup _{t \in[0, T]}|\delta f(t, x(t), e(t))| \\
& \leq \frac{K_{\alpha}}{\alpha}\left[\mathbf{f}_{e}\left(\mathbf{x}_{p}, \mathbf{e}_{p}\right) \mathbf{e}_{p}+\frac{1}{2} \mathbf{f}_{x x}\left(\mathbf{x}_{p}, 0\right) \mathbf{x}_{p}^{2}\right],
\end{aligned}
$$

in which we used (62) and (67) to derive the second inequality. Then, note that by definition of (59), and by exploiting the linearity of the function $\Psi$ defined in (52), we have

$$
\begin{aligned}
\mathfrak{O}\left[x_{a}, e_{a}\right]_{x}(t)-\mathfrak{O}\left[x_{b}, e_{b}\right]_{x}(t) & =\Psi\left(F, \delta f\left(t, x_{a}(t), e_{a}(t)\right)\right)-\Psi\left(F, \delta f\left(t, x_{b}(t), e_{b}(t)\right)\right) \\
& =\Psi\left(F, \delta f\left(t, x_{a}(t), e_{a}(t)\right)-\delta f\left(t, x_{b}(t), e_{b}(t)\right)\right)
\end{aligned}
$$

and therefore, by using (55), (68), and $\sup _{s \in[0, T]}\left|x_{a}(s)-x_{b}(s)\right| \leq 2 \mathbf{x}_{p}$, we obtain

$$
\begin{aligned}
& \left|\mathfrak{O}\left[x_{a}, e_{a}\right]_{x}(t)-\mathfrak{O}\left[x_{b}, e_{b}\right]_{x}(t)\right| \leq \\
& \quad \frac{K_{\alpha}}{\alpha}\left[\mathbf{f}_{e x}\left(\mathbf{x}_{p}, \mathbf{e}_{p}\right) \mathbf{e}_{p}+2 \mathbf{f}_{x x}\left(\mathbf{x}_{p}, 0\right) \mathbf{x}_{p}\right] \sup _{s \in[0, T]}\left|x_{a}(s)-x_{b}(s)\right|+\frac{K_{\phi}}{\alpha} \mathbf{f}_{e}\left(\mathbf{x}_{p}, \mathbf{e}_{p}\right) \sup _{s \in[0, T]}\left|e_{a}(s)-e_{b}(s)\right| .
\end{aligned}
$$

Similarly for $\mathfrak{O}[x, e]_{e}$ in (60), with

$$
\mathfrak{O}[x, e]_{e}(t)=\Psi(-\sigma, q(t, x, e))+\Psi(-\sigma, \eta[x, e](t))
$$

and (56) where

$$
\underline{P}_{\chi}=\bar{P}_{\chi}=1, \quad \alpha=\sigma, \quad K_{\alpha}=1, \quad K_{\alpha, 2}=\sqrt{\frac{T(1+\exp (-\sigma T))}{2(1-\exp (-\sigma T))}}
$$

we obtain

$$
\sup _{t \in[0, T]}\left|\mathfrak{O}[x, e]_{e}(t)\right| \leq \frac{K_{\alpha, 2}}{\sqrt{\sigma}}\left[\sqrt{\int_{0}^{T}|q(t, x(t), e(t))|^{2} d t}+\sqrt{\int_{0}^{T}|\eta[x, e](t)|^{2} d t}\right] .
$$

Then (39) gives

$$
\sup _{t \in[0, T]}\left|\mathfrak{O}[x, e]_{e}(t)\right| \leq \frac{2 K_{\alpha, 2}}{\sqrt{\sigma}} \sqrt{\int_{0}^{T}|q(t, x(t), e(t))|^{2} d t} .
$$

By using $\int_{0}^{T}|q(t)|^{2} d t \leq T \sup _{t \in[0, T]}|q(t)|^{2}$ and bound (64), we finally obtain

$$
\sup _{t \in[0, T]}\left|\mathfrak{O}[x, e]_{e}(t)\right| \leq \beta(\sigma) \mathbf{q}\left(\mathbf{x}_{p}, \mathbf{e}_{p}\right) .
$$

in which $\beta$ is defined as

$$
\beta(\sigma):=\sqrt{\frac{2 T(1+\exp (-\sigma T))}{\sigma(1-\exp (-\sigma T))}} .
$$

Note that $\beta$ is a continuous strictly decreasing positive function for $\sigma>0$. In particular, $\lim _{\sigma \rightarrow \infty} \beta(\sigma)=0$.

Finally, as done to obtain inequality (70), we can use linearity of the operator $\Psi$ and inequalities (71) and (65) to derive

$$
\begin{aligned}
\sup _{t \in[0, T]} \mid \mathfrak{O}\left[x_{a}, e_{a}\right]_{e}(t) & -\mathfrak{O}\left[x_{b}, e_{b}\right]_{e}(t) \mid \leq \\
& \beta(\sigma)\left(\mathbf{q}_{e}\left(\mathbf{x}_{p}, \mathbf{e}_{p}\right) \sup _{s \in[0, T]}\left|e_{a}(s)-e_{b}(s)\right|+\mathbf{q}_{x}\left(\mathbf{x}_{p}, \mathbf{e}_{p}\right) \sup _{s \in[0, T]}\left|x_{a}(s)-x_{b}(s)\right|\right) .
\end{aligned}
$$


In conclusion, with (69) and (71), we have established that, if $\mathbf{x}_{p}, \mathbf{e}_{p}$ and $\sigma$ satisfy

$$
\begin{aligned}
\frac{K_{\alpha}}{\alpha}\left[\mathbf{f}_{e}\left(\mathbf{x}_{p}, \mathbf{e}_{p}\right) \mathbf{e}_{p}+\frac{1}{2} \mathbf{f}_{x x}\left(\mathbf{x}_{p}, 0\right) \mathbf{x}_{p}^{2}\right] & \leq \mathbf{x}_{p}, \\
\beta(\sigma) \mathbf{q}\left(\mathbf{x}_{p}, \mathbf{e}_{p}\right) & \leq \mathbf{e}_{p},
\end{aligned}
$$

with $\beta$ defined in (72), and if $(x, e)$ are $T$-periodic continuous functions satisfying (62), then $\left(\mathfrak{O}[x, e]_{x}, \mathfrak{O}[x, e]_{e}\right)$ are $T$-periodic continuous functions satisfying

$$
\sup _{t \in[0, T]}\left|\mathfrak{O}[x, e]_{x}(t)\right| \leq \mathbf{x}_{p}, \quad \sup _{t \in[0, T]}\left|\mathfrak{O}[x, e]_{e}(t)\right| \leq \mathbf{e}_{p}
$$

Similarly, with (70) and (73), we have established that, if $\left(x_{a}, e_{a}\right)$ and $\left(x_{b}, e_{b}\right)$ are $T$-periodic continuous functions satisfying (62),

$$
\left(\begin{array}{l}
\left|\mathfrak{O}\left[x_{a}, e_{a}\right]_{x}(t)-\mathfrak{O}\left[x_{b}, e_{b}\right]_{x}(t)\right| \\
\left|\mathfrak{O}\left[x_{a}, e_{a}\right]_{e}(t)-\mathfrak{O}\left[x_{b}, e_{b}\right]_{e}(t)\right|
\end{array}\right) \leq \mathfrak{M}\left(\mathbf{x}_{p}, \mathbf{e}_{p}, \sigma\right)\left(\begin{array}{l}
\sup _{s \in[0, T]}\left|x_{a}(s)-x_{b}(s)\right| \\
\sup _{s \in[0, T]}\left|e_{a}(s)-e_{b}(s)\right|
\end{array}\right)
$$

with the notation

$$
\mathfrak{M}\left(\mathbf{x}_{p}, \mathbf{e}_{p}, \sigma\right):=\left(\begin{array}{cc}
\mathfrak{M}_{x x}\left(\mathbf{x}_{p}, \mathbf{e}_{p}, \sigma\right) & \mathfrak{M}_{x e}\left(\mathbf{x}_{p}, \mathbf{e}_{p}, \sigma\right) \\
\mathfrak{M}_{e x}\left(\mathbf{x}_{p}, \mathbf{e}_{p}, \sigma\right) & \mathfrak{M}_{e e}\left(\mathbf{x}_{p}, \mathbf{e}_{p}, \sigma\right)
\end{array}\right)
$$

where $\mathfrak{M}$ is the following matrix with strictly positive entries

$$
\begin{array}{ll}
\mathfrak{M}_{x x}\left(\mathbf{x}_{p}, \mathbf{e}_{p}, \sigma\right)=\frac{K_{\phi}}{\alpha}\left[\mathbf{f}_{e x}\left(\mathbf{x}_{p}, \mathbf{e}_{p}\right) \mathbf{e}_{p}+2 \mathbf{f}_{x x}\left(\mathbf{x}_{p}, 0\right) \mathbf{x}_{p}\right], & \mathfrak{M}_{x e}\left(\mathbf{x}_{p}, \mathbf{e}_{p}, \sigma\right)=\frac{K_{\phi}}{\alpha} \mathbf{f}_{e}\left(\mathbf{x}_{p}, \mathbf{e}_{p}\right) \\
\mathfrak{M}_{e x}\left(\mathbf{x}_{p}, \mathbf{e}_{p}, \sigma\right)=\beta(\sigma) \mathbf{q}_{x}\left(\mathbf{x}_{p}, \mathbf{e}_{p}\right) \quad, & \mathfrak{M}_{e e}\left(\mathbf{x}_{p}, \mathbf{e}_{p}, \sigma\right)=\beta(\sigma) \mathbf{q}_{e}\left(\mathbf{x}_{p}, \mathbf{e}_{p}\right)
\end{array}
$$

It follows from Perron-Frobenius theorem that there exist strictly positive real numbers $p_{x}\left(\mathbf{x}_{p}, \mathbf{e}_{p}, \sigma\right)$, $p_{e}\left(\mathbf{x}_{p}, \mathbf{e}_{p}, \sigma\right)$ and $\gamma\left(\mathbf{x}_{p}, \mathbf{e}_{p}, \sigma\right)$, depending on $\left(\mathbf{x}_{p}, \mathbf{e}_{p}, \sigma\right)$ and satisfying

$$
\mathfrak{M}^{\top}\left(\begin{array}{l}
p_{x} \\
p_{e}
\end{array}\right)=\gamma\left(\begin{array}{l}
p_{x} \\
p_{e}
\end{array}\right)
$$

Here, $\gamma$ is a simple eigenvalue of $\mathfrak{M}$ and the spectral radius of this matrix. It is strictly smaller than 1 if and only if we have

$$
\begin{aligned}
& 1-\left[\mathfrak{M}_{x x}+\mathfrak{M}_{e e}\right]+\left[\mathfrak{M}_{x x} \mathfrak{M}_{e e}-\mathfrak{M}_{x e} \mathfrak{M}_{e x}\right]>0 \\
& 1-\left[\mathfrak{M}_{x x} \mathfrak{M}_{e e}-\mathfrak{M}_{x e} \mathfrak{M}_{e x}\right]>0
\end{aligned}
$$

i.e.

$$
\begin{aligned}
& 1>\frac{K_{\alpha}}{\alpha}\left[\mathbf{f}_{e x}\left(\mathbf{x}_{p}, \mathbf{e}_{p}\right) \mathbf{e}_{p}+2 \mathbf{f}_{x x}\left(\mathbf{x}_{p}, 0\right) \mathbf{x}_{p}\right]+\beta(\sigma) \mathbf{q}_{e}\left(\mathbf{x}_{p}, \mathbf{e}_{p}\right) \\
& +\frac{K_{\alpha} \beta(\sigma)}{\alpha}\left(\mathbf{f}_{e}\left(\mathbf{x}_{p}, \mathbf{e}_{p}\right) \mathbf{q}_{x}\left(\mathbf{x}_{p}, \mathbf{e}_{p}\right)-\left[\mathbf{f}_{e x}\left(\mathbf{x}_{p}, \mathbf{e}_{p}\right) \mathbf{e}_{p}+2 \mathbf{f}_{x x}\left(\mathbf{x}_{p}, 0\right) \mathbf{x}_{p}\right] \mathbf{q}_{e}\left(\mathbf{x}_{p}, \mathbf{e}_{p}\right)\right), \\
& 1>\frac{K_{\alpha} \beta(\sigma)}{\alpha}\left(\mathbf{f}_{e}\left(\mathbf{x}_{p}, \mathbf{e}_{p}\right) \mathbf{q}_{x}\left(\mathbf{x}_{p}, \mathbf{e}_{p}\right)-\left[\mathbf{f}_{e x}\left(\mathbf{x}_{p}, \mathbf{e}_{p}\right) \mathbf{e}_{p}+2 \mathbf{f}_{x x}\left(\mathbf{x}_{p}, 0\right) \mathbf{x}_{p}\right] \mathbf{q}_{e}\left(\mathbf{x}_{p}, \mathbf{e}_{p}\right)\right)
\end{aligned}
$$

With this at hand, (70), (73) and (77) give

$$
\begin{gathered}
p_{x}\left(\mathbf{x}_{p}, \mathbf{e}_{p}, \sigma\right) \sup _{t \in[0, T]}\left|\mathfrak{O}\left[x_{a}, e_{a}\right]_{x}(t)-\mathfrak{O}\left[x_{b}, e_{b}\right]_{x}(t)\right|+p_{e}\left(\mathbf{x}_{p}, \mathbf{e}_{p}, \sigma\right) \sup _{t \in[0, T]}\left|\mathfrak{O}\left[x_{a}, e_{a}\right]_{e}(t)-\mathfrak{O}\left[x_{b}, e_{b}\right]_{e}(t)\right| \\
\leq \gamma\left(\mathbf{x}_{p}, \mathbf{e}_{p}, \sigma\right)\left[p_{x}\left(\mathbf{x}_{p}, \mathbf{e}_{p}, \sigma\right) \sup _{t \in[0, T]}\left|x_{a}(t)-x_{b}(t)\right|+p_{e}\left(\mathbf{x}_{p}, \mathbf{e}_{p}, \sigma\right) \sup _{t \in[0, T]}\left|e_{a}(t)-e_{b}(t)\right|\right] .
\end{gathered}
$$

Now, let $\mathscr{B}_{\mathbf{x}_{p}, \mathbf{e}_{p}}\left(\mathbb{R}^{n+1}\right)$ denote the closed subset of $\mathscr{C}_{T}^{0}\left(\mathbb{R}^{n+1}\right)$ defined as

$$
\mathscr{B}_{\mathbf{x}_{p}, \mathbf{e}_{p}}\left(\mathbb{R}^{n+1}\right):=\left\{(x, e) \in \mathscr{C}_{T}^{0}\left(\mathbb{R}^{n+1}\right): \sup _{t \in[0, T]}|x(t)| \leq \mathbf{x}_{p}, \sup _{t \in[0, T]}|e(t)| \leq \mathbf{e}_{p}\right\} .
$$


This set $\mathscr{B}_{\mathbf{x}_{p}, \mathbf{e}_{p}}\left(\mathbb{R}^{n+1}\right)$ equipped with the norm

$$
\|(x, e)\|:=p_{x}\left(\mathbf{x}_{p}, \mathbf{e}_{p}, \sigma\right) \sup _{t \in[0, T]}|x(t)|+p_{e}\left(\mathbf{x}_{p}, \mathbf{e}_{p}, \sigma\right) \sup _{t \in[0, T]}|e(t)|
$$

is a complete metric space. Assuming for the time being (see below) there exists a triple $\left(\mathbf{x}_{p}, \mathbf{e}_{p}, \sigma_{p}^{\star}\right)$ satisfying (74) and (78), we have established that, for any $\sigma>\sigma_{p}^{\star}$, we have the following properties.

1. The function $(x, e) \mapsto\left(\mathfrak{O}[x, e]_{x}, \mathfrak{O}[x, e]_{e}\right)$ maps a function in $\mathscr{B}_{\mathbf{x}_{p}, \mathbf{e}_{p}}\left(\mathbb{R}^{n+1}\right)$ into a function in $\mathscr{B}_{\mathbf{x}_{p}, \mathbf{e}_{p}}\left(\mathbb{R}^{n+1}\right)$, since (74) implies (75).

2. The function $(x, e) \mapsto\left(\mathfrak{O}[x, e]_{x}, \mathfrak{O}[x, e]_{e}\right)$ is a contraction, the gain $\gamma\left(\mathbf{x}_{p}, \mathbf{e}_{p}, \sigma\right)$ in (79) being strictly smaller than 1 when (78) holds.

We conclude, from the Banach fixed point theorem, that there exists a fixed point $\left(x_{p}, e_{p}\right)$ in $\mathscr{B}_{\mathbf{x}_{p}, \mathbf{e}_{p}}\left(\mathbb{R}^{n+1}\right)$, namely there exist $x_{p}$ and $e_{p}$ satisfying $\mathfrak{O}\left[x_{p}, e_{p}\right]_{x}=x_{p}$ and $\mathfrak{O}\left[x_{p}, e_{p}\right]_{e}=e_{p}$. In particular, $x_{p}, e_{p}$ are $C^{0}$, $T$-periodic, satisfy

$$
\sup _{t \in[0, T]}\left|x_{p}(t)\right| \leq \mathbf{x}_{p}, \quad \sup _{t \in[0, T]}\left|e_{p}(t)\right| \leq \mathbf{e}_{p}
$$

and are solution of

$$
\begin{aligned}
& \dot{x}_{p}=F(t) x_{p}+\delta f\left(t, x_{p}, e_{p}\right), \\
& \dot{e}_{p}=-\sigma e_{p}+\mu M^{\top} N_{z} \zeta_{p}(t)+q\left(t, x_{p}, e_{p}\right)
\end{aligned}
$$

where $\zeta_{p}$ is the unique $T$-periodic solution of

$$
\dot{\zeta}_{p}=\left[\Phi-\mu M M^{\top} N_{z}\right] \zeta_{p}-M q\left(t, x_{p}(t), e_{p}(t)\right) .
$$

as stated by Lemma 3. Also because of $F, \delta f$ and $q$ are $C^{1}$, the periodic solution $\left(x_{p}, e_{p}\right)$ is $C^{2}$.

In order to determine a bound for $\zeta_{p}$, we apply Lemma 3 and inequality (40) to the solution $\zeta_{p}$ defined in (81c). To this end, we need a bound for $\dot{q}$. It can be expressed as

$$
\begin{aligned}
\dot{q}\left(t, x_{p}(t), e_{p}(t)\right)=\frac{\partial q}{\partial t}\left(t, x_{p}(t), e_{p}(t)\right) & +\frac{\partial q}{\partial x}\left(t, x_{p}(t), e_{p}(t)\right) f\left(t, x_{p}(t), e_{p}(t)\right) \\
& +\frac{\partial q}{\partial e}\left(t, x_{p}(t), e_{p}(t)\right)\left[-\sigma e_{p}(t)+\mu M^{\top} N_{z} \zeta_{p}(t)+q\left(t, x_{p}(t), e_{p}(t)\right)\right] .
\end{aligned}
$$

As a consequence, the previous expression of $\dot{q}$ yields, using (39), and the bounds (80) for $x_{p}, e_{p}$,

$$
\begin{aligned}
\int_{0}^{T}\left|\dot{q}_{p}(t)\right|^{2} d t \leq & 3 T \mathbf{q}_{t}\left(\mathbf{x}_{p}, \mathbf{e}_{p}\right)^{2}+3 T \mathbf{q}_{x}\left(\mathbf{x}_{p}, \mathbf{e}_{p}\right)^{2} \mathbf{f}\left(\mathbf{x}_{p}, \mathbf{e}_{p}\right)^{2} \\
& +9 \mathbf{q}_{e}\left(\mathbf{x}_{p}, \mathbf{e}_{p}\right)^{2} \int_{0}^{T}\left[\sigma^{2} e_{p}(t)^{2}+\mu^{2}\left|M^{\top} N_{z} \zeta_{p}(t)\right|^{2}+\left|q\left(t, x_{p}(t), e_{p}(t)\right)\right|^{2}\right] d t \\
\leq & 3 T \mathbf{q}_{t}\left(\mathbf{x}_{p}, \mathbf{e}_{p}\right)^{2}+3 T \mathbf{q}_{x}\left(\mathbf{x}_{p}, \mathbf{e}_{p}\right)^{2} \mathbf{f}\left(\mathbf{x}_{p}, \mathbf{e}_{p}\right)^{2} \\
& +9 \mathbf{q}_{e}\left(\mathbf{x}_{p}, \mathbf{e}_{p}\right)^{2}\left[\sigma^{2} \int_{0}^{T} e_{p}(t)^{2} d t+2 \int_{0}^{T}\left|q\left(t, x_{p}(t), e_{p}(t)\right)\right|^{2} d t\right]
\end{aligned}
$$

On another hand, we obtain, by integration and using (39) again,

$$
\begin{aligned}
0= & \frac{e_{p}(T)^{2}-e_{p}(0)^{2}}{2} \leq \\
& \leq-\sigma \int_{0}^{T} e_{p}(t)^{2} d t+\mu \int_{0}^{T} M^{\top} N_{z} \zeta_{p}(t) e_{p}(t) d t+\int_{0}^{T} q\left(t, x_{p}(t), e_{p}(t)\right) e_{p}(t) d t \\
& \leq-\frac{\sigma}{2} \int_{0}^{T} e_{p}(t)^{2} d t+\frac{1}{\sigma} \mu^{2} \int_{0}^{T}\left|M^{\top} N_{z} \zeta_{p}(t)\right|^{2} d t+\frac{1}{\sigma} \int_{0}^{T}\left|q\left(t, x_{p}(t), e_{p}(t)\right)\right|^{2} d t \\
& \leq-\frac{\sigma}{2} \int_{0}^{T} e_{p}(t)^{2} d t+\frac{2}{\sigma} \int_{0}^{T}\left|q\left(t, x_{p}(t), e_{p}(t)\right)\right|^{2} d t
\end{aligned}
$$

and therefore

$$
\begin{aligned}
\int_{0}^{T}\left|\dot{q}_{p}(s)\right|^{2} d s & \leq 3 T\left[\mathbf{q}_{t}\left(\mathbf{x}_{p}, \mathbf{e}_{p}\right)^{2}+\mathbf{q}_{x}\left(\mathbf{x}_{p}, \mathbf{e}_{p}\right)^{2} \mathbf{f}\left(\mathbf{x}_{p}, \mathbf{e}_{p}\right)^{2}\right]+54 \mathbf{q}_{e}\left(\mathbf{x}_{p}, \mathbf{e}_{p}\right)^{2} \int_{0}^{T}\left|q\left(t, x_{p}(t), e_{p}(t)\right)\right|^{2} d t \\
& \leq 3 T\left[\mathbf{q}_{t}\left(\mathbf{x}_{p}, \mathbf{e}_{p}\right)^{2}+\mathbf{q}_{x}\left(\mathbf{x}_{p}, \mathbf{e}_{p}\right)^{2} \mathbf{f}\left(\mathbf{x}_{p}, \mathbf{e}_{p}\right)^{2}\right]+54 \mathbf{q}_{e}\left(\mathbf{x}_{p}, \mathbf{e}_{p}\right)^{2} T \mathbf{q}\left(\mathbf{x}_{p}, \mathbf{e}_{p}\right)^{2}
\end{aligned}
$$


By combining this inequality with (40) and (64), we finally obtain an expression for the bound $\boldsymbol{\zeta}_{p}$ in inequality (46c), that is,

$$
\zeta_{p}^{2}(\mu):=\left[\frac{T}{\mu}+\kappa_{0}+54 \kappa_{1} \mathbf{q}_{e}\left(\mathbf{x}_{p}, \mathbf{e}_{p}\right)^{2}\right] \mathbf{q}\left(\mathbf{x}_{p}, \mathbf{e}_{p}\right)^{2}+3 \kappa_{1}\left[\mathbf{q}_{t}\left(\mathbf{x}_{p}, \mathbf{e}_{p}\right)^{2}+\mathbf{q}_{x}\left(\mathbf{x}_{p}, \mathbf{e}_{p}\right)^{2} \mathbf{f}\left(\mathbf{x}_{p}, \mathbf{e}_{p}\right)^{2}\right]
$$

with $\kappa_{0}, \kappa_{1}$ given by Lemma 2. It is independent of $\sigma$ and of $\mu$ by noting that it is a decreasing function of $\mu$ for $\mu \geq 1$, that is, select $\zeta_{p}(1)$ in the statement of the Theorem.

Now, inequalities (80) are parts of the inequalities (46a) and (46b). On another hand, with (55) and (67), we obtain

$$
\sup _{t \in[0, T]}\left|x_{p}(t)\right| \leq \frac{K_{\alpha}}{\alpha}\left[\mathbf{f}_{e}\left(\mathbf{x}_{p}, \mathbf{e}_{p}\right) \sup _{t \in[0, T]}\left|e_{p}(t)\right|+\frac{1}{2} \mathbf{f}_{x x}\left(\mathbf{x}_{p}, 0\right) \mathbf{x}_{p} \sup _{t \in[0, T]}\left|x_{p}(t)\right|\right]
$$

which gives

$$
\sup _{t \in[0, T]}\left|x_{p}(t)\right| \leq \frac{K_{\alpha} \mathbf{f}_{e}\left(\mathbf{x}_{p}, \mathbf{e}_{p}\right)}{\alpha-\frac{1}{2} K_{\alpha} \mathbf{f}_{x x}\left(\mathbf{x}_{p}, 0\right) \mathbf{x}_{p}} \sup _{t \in[0, T]}\left|e_{p}(t)\right|,
$$

where the denominator of the right hand side is strictly positive according to (74a). Now recall that by definition of (7a) and of the matrices $M, N_{z}$, we have $\sqrt{M^{\top} N_{z} M} \leq \bar{N}_{z}$. As a consequence, inequalities (55) and (46c) give

$$
\begin{aligned}
\sup _{t \in[0, T]}\left|e_{p}(t)\right| & \leq \frac{1}{\sigma}\left[\sup _{t \in[0, T]} q\left(t, x_{p}(t), e_{p}(t)\right)+\sup _{t \in[0, T]} M^{\top} N_{z} \zeta_{p}(t)\right] \\
& \leq \frac{1}{\sigma}\left[\mathbf{q}\left(\mathbf{x}_{p}, \mathbf{e}_{p}\right)+\sqrt{M^{\top} N_{z} M} \sup _{t \in[0, T]} \sqrt{\zeta_{p}(t)^{\top} N_{z} \zeta_{p}(t)}\right] \\
& \leq \frac{1}{\sigma}\left(\mathbf{q}\left(\mathbf{x}_{p}, \mathbf{e}_{p}\right)+\bar{N}_{z} \boldsymbol{\zeta}_{p}\right)
\end{aligned}
$$

This yields the remaining parts of of the inequalities (46a) and (46b) with

$$
\mathbf{r}_{e}:=\mathbf{q}\left(\mathbf{x}_{p}, \mathbf{e}_{p}\right)+\bar{N}_{z} \boldsymbol{\zeta}_{p}, \quad \mathbf{r}_{x}:=\frac{K_{\alpha} \mathbf{f}_{e}\left(\mathbf{x}_{p}, \mathbf{e}_{p}\right)}{\alpha-\frac{1}{2} K_{\alpha} \mathbf{f}_{x x}\left(\mathbf{x}_{p}, 0\right) \mathbf{x}_{p}} \mathbf{r}_{e} .
$$

Finally, to complete the proof, we need to show that it does exist a triple of positive real numbers $\left(\mathbf{x}_{p}, \mathbf{e}_{p}, \sigma_{p}^{\star}\right)$ satisfying (74) and (78), i.e.

$\mathbf{x}_{p} \geq \frac{K_{\alpha}}{\alpha}\left[\mathbf{f}_{e}\left(\mathbf{x}_{p}, \mathbf{e}_{p}\right) \mathbf{e}_{p}+\frac{1}{2} \mathbf{f}_{x x}\left(\mathbf{x}_{p}, 0\right) \mathbf{x}_{p}^{2}\right]$,

$\mathbf{e}_{p} \geq \beta(\sigma) \mathbf{q}\left(\mathbf{x}_{p}, \mathbf{e}_{p}\right)$,

$1>\frac{K_{\alpha}}{\alpha}\left[\mathbf{f}_{e x}\left(\mathbf{x}_{p}, \mathbf{e}_{p}\right) \mathbf{e}_{p}+2 \mathbf{f}_{x x}\left(\mathbf{x}_{p}, 0\right) \mathbf{x}_{p}\right]+\beta(\sigma) \mathbf{q}_{e}\left(\mathbf{x}_{p}, \mathbf{e}_{p}\right)$

$$
+\frac{K_{\alpha} \beta(\sigma)}{\alpha}\left(\mathbf{f}_{e}\left(\mathbf{x}_{p}, \mathbf{e}_{p}\right) \mathbf{q}_{x}\left(\mathbf{x}_{p}, \mathbf{e}_{p}\right)-\left[\mathbf{f}_{e x}\left(\mathbf{x}_{p}, \mathbf{e}_{p}\right) \mathbf{e}_{p}+2 \mathbf{f}_{x x}\left(\mathbf{x}_{p}, 0\right) \mathbf{x}_{p}\right] \mathbf{q}_{e}\left(\mathbf{x}_{p}, \mathbf{e}_{p}\right)\right),
$$

$1>\frac{K_{\alpha} \beta(\sigma)}{\alpha}\left(\mathbf{f}_{e}\left(\mathbf{x}_{p}, \mathbf{e}_{p}\right) \mathbf{q}_{x}\left(\mathbf{x}_{p}, \mathbf{e}_{p}\right)-\left[\mathbf{f}_{e x}\left(\mathbf{x}_{p}, \mathbf{e}_{p}\right) \mathbf{e}_{p}+2 \mathbf{f}_{x x}\left(\mathbf{x}_{p}, 0\right) \mathbf{x}_{p}\right] \mathbf{q}_{e}\left(\mathbf{x}_{p}, \mathbf{e}_{p}\right)\right)$.

Since the function $\beta$ is continuous, strictly decreasing and tends to 0 as $\sigma$ tends to infinity, it is sufficient to find $\mathbf{x}_{p}$ and $\mathbf{e}_{p}$ satisfying :

$$
\begin{gathered}
\mathbf{x}_{p}>\frac{K_{\alpha}}{\alpha}\left[\mathbf{f}_{e}\left(\mathbf{x}_{p}, \mathbf{e}_{p}\right) \mathbf{e}_{p}+\frac{1}{2} \mathbf{f}_{x x}\left(\mathbf{x}_{p}, 0\right) \mathbf{x}_{p}^{2}\right], \\
1>\frac{K_{\alpha}}{\alpha}\left[\mathbf{f}_{e x}\left(\mathbf{x}_{p}, \mathbf{e}_{p}\right) \mathbf{e}_{p}+2 \mathbf{f}_{x x}\left(\mathbf{x}_{p}, 0\right) \mathbf{x}_{p}\right] .
\end{gathered}
$$


Since the bounding functions $\mathbf{f}_{\bullet}$ are increasing in each of their arguments, we choose

$$
\mathbf{x}_{p}<\mathbf{x}_{p *} \quad, \quad \mathbf{e}_{p} \leq \mathbf{e}_{p *}\left(\mathbf{x}_{p}\right),
$$

where $\mathbf{x}_{p *}$ is a strictly positive real number satisfying

$$
\mathbf{x}_{p *} \mathbf{f}_{x x}\left(\mathbf{x}_{p *}, 0\right)<\frac{\alpha}{2 K_{\alpha}}
$$

and $\mathbf{e}_{p *}\left(\mathbf{x}_{p}\right)$ is a strictly positive real number satisfying

$$
\begin{aligned}
& \mathbf{e}_{p *}\left(\mathbf{x}_{p}\right) \mathbf{f}_{e}\left(\mathbf{x}_{p}, \mathbf{e}_{p *}\left(\mathbf{x}_{p}\right)\right) \leq \frac{\alpha}{K_{\alpha}} \mathbf{x}_{p}-\frac{1}{2} \mathbf{f}_{x x}\left(\mathbf{x}_{p}, 0\right) \mathbf{x}_{p}^{2}, \\
& \mathbf{e}_{p *}\left(\mathbf{x}_{p}\right) \mathbf{f}_{e x}\left(\mathbf{x}_{p}, \mathbf{e}_{p *}\left(\mathbf{x}_{p}\right)\right)<\frac{\alpha}{K_{\alpha}}-2 \mathbf{x}_{p} \mathbf{f}_{x x}\left(\mathbf{x}_{p}, 0\right) .
\end{aligned}
$$

In this way (83) holds and it remains to find $\sigma_{p}^{\star}$ large enough to satisfy the following 3 inequalities

$$
\begin{aligned}
& \beta\left(\sigma_{p}^{\star}\right) \leq \frac{\mathbf{e}_{p}}{\mathbf{q}\left(\mathbf{x}_{p}, \mathbf{e}_{p}\right)} \\
& \beta\left(\sigma_{p}^{\star}\right)<\frac{1-\frac{K \alpha}{\alpha}\left[\mathbf{f}_{e x}\left(\mathbf{x}_{p}, \mathbf{e}_{p}\right) \mathbf{e}_{p}+2 \mathbf{f}_{x x}\left(\mathbf{x}_{p}, 0\right) \mathbf{x}_{p}\right]}{\mathbf{q}_{e}\left(\mathbf{x}_{p}, \mathbf{e}_{p}\right)+\frac{K \alpha}{\alpha}\left(\mathbf{f}_{e}\left(\mathbf{x}_{p}, \mathbf{e}_{p}\right) \mathbf{q}_{x}\left(\mathbf{x}_{p}, \mathbf{e}_{p}\right)-\left[\mathbf{f}_{e x}\left(\mathbf{x}_{p}, \mathbf{e}_{p}\right) \mathbf{e}_{p}+2 \mathbf{f}_{x x}\left(\mathbf{x}_{p}, 0\right) \mathbf{x}_{p}\right] \mathbf{q}_{e}\left(\mathbf{x}_{p}, \mathbf{e}_{p}\right)\right)} \\
& \beta\left(\sigma_{p}^{\star}\right)<\frac{\alpha}{K_{\alpha}\left(\mathbf{f}_{e}\left(\mathbf{x}_{p}, \mathbf{e}_{p}\right) \mathbf{q}_{x}\left(\mathbf{x}_{p}, \mathbf{e}_{p}\right)-\left[\mathbf{f}_{e x}\left(\mathbf{x}_{p}, \mathbf{e}_{p}\right) \mathbf{e}_{p}+2 \mathbf{f}_{x x}\left(\mathbf{x}_{p}, 0\right) \mathbf{x}_{p}\right] \mathbf{q}_{e}\left(\mathbf{x}_{p}, \mathbf{e}_{p}\right)\right)} .
\end{aligned}
$$

Note $n_{o}$ plays no role in this process implying that the triple $\left(\mathbf{x}_{p}, \mathbf{e}_{p}, \sigma_{p}^{\star}\right)$ does not depend on $n_{o}$.

Remark 2 We stress the fact that $\mathbf{x}_{p}$ can be chosen arbitrarily in $] 0, \mathbf{x}_{p *}\left[\right.$. Then $\mathbf{x}_{p}$ being fixed, $\mathbf{e}_{p}$ can be chosen arbitrarily in $\left.] 0, \mathbf{e}_{p *}\left(\mathbf{x}_{p}\right)\right]$. And finally, $\mathbf{x}_{p}$ and $\mathbf{e}_{p}$ being fixed, $\sigma_{p}^{\star}$ is chosen to satisfy the 3 inequalities in (87)

\section{A.4 Exponential Stability of the Periodic Solution}

Proposition 2 For any triplet $\left(\bar{P}_{x}, \underline{P}_{x}, \alpha\right)$, there exist strictly positive real numbers $\mathbf{x}_{a}, \mathbf{e}_{a}>0$ (independent of $n_{o}$ ) and, for any $\zeta_{a}>0$ and $\mu \geq 1$, there exists $\sigma_{a}^{\star}>0$ (independent of $n_{o}$ ) such that, if system (28), with $\sigma>\sigma_{a}^{\star}, f \in \mathscr{F}\left(\mathbf{x}_{a}, \mathbf{e}_{a}, \bar{P}_{x}, \underline{P}_{x}, \alpha\right)$ and $q \in \mathscr{Q}\left(\mathbf{x}_{a}, \mathbf{e}_{a}\right)$, admits a T-periodic solution $\left(x_{p}, e_{p}, z_{p}\right)$ satisfying

$$
\sup _{t \in[0, T]}\left|x_{p}(t)\right| \leq \mathbf{x}_{a}, \sup _{t \in[0, T]}\left|e_{p}(t)\right| \leq \mathbf{e}_{a}, \quad \sup _{t \in[0, T]} \sqrt{\zeta_{p}(0)^{\top} N_{z} \zeta_{p}(0)} \leq \boldsymbol{\zeta}_{a},
$$

then, such periodic solution is exponentially stable with a domain of attraction containing the set

$\mathscr{N}_{\zeta}\left(\mathbf{x}_{a}, \mathbf{e}_{a}, \boldsymbol{\zeta}_{a}\right)=\left\{(x, e, \zeta):\left|x-x_{p}(0)\right| \leq 3 \mathbf{x}_{a},\left|e-e_{p}(0)\right| \leq 3 \mathbf{e}_{a}, \sqrt{\left(\zeta-\zeta_{p}(0)\right)^{\top} N_{z}\left(\zeta-\zeta_{p}(0)\right)} \leq 3 \boldsymbol{\zeta}_{a}\right\}$.

Proof The assumed existence of a periodic solution $\left(x_{p}, e_{p}, \zeta_{p}\right)$ satisfying (88) for system (28) allows us to consider the following change of coordinates

$$
(x, e, \zeta) \mapsto(\tilde{x}, \tilde{e}, \tilde{\zeta}):=\left(x-x_{p}(t), e-e_{p}(t), \zeta-\zeta_{p}(t)\right)
$$

System (28) is transformed into

$$
\begin{aligned}
\dot{\tilde{x}} & =F_{p}(t) \tilde{x}+\delta \tilde{f}(t, \tilde{x}, \tilde{e}) \\
\dot{\tilde{e}} & =\tilde{q}(t, \tilde{x}, \tilde{e})-\sigma \tilde{e}+\mu M^{\top} N_{z} \tilde{\zeta} \\
\dot{\zeta} & =\left(\Phi-\mu M M^{\top} N_{z}\right) \tilde{\zeta}-M \tilde{q}(t, \tilde{x}, \tilde{e})
\end{aligned}
$$


with the definitions

$$
\begin{aligned}
F_{p}(t) & :=\frac{\partial f}{\partial x}\left(t, x_{p}(t), e_{p}(t)\right), \\
\delta \tilde{f}(t, \tilde{x}, \tilde{e}) & :=\tilde{f}(t, \tilde{x}, \tilde{e})-F_{p}(t) \tilde{x}, \\
\tilde{f}(t, \tilde{x}, \tilde{e}) & :=f(t, x, e)-f\left(t, x_{p}(t), e_{p}(t)\right), \\
\tilde{q}(t, \tilde{x}, \tilde{e}) & :=q(t, x, e)-q\left(t, x_{p}(t), e_{p}(t)\right) .
\end{aligned}
$$

Exponential stability of the origin for the system (90) can be established from the first order approximation. But we want to establish not only exponential stability but also that the domain of attraction contains $\mathscr{N}_{\zeta}\left(\mathbf{x}_{a}, \mathbf{e}_{a}, \boldsymbol{\zeta}_{a}\right)$ defined in (89). So instead we go with a Lyapunov analysis.

First, we define $\mathbf{x}_{a}, \mathbf{e}_{a}$ and $\sigma_{a}^{\star}$ via a set of inequality. To this end, to any pair $\left(\mathbf{x}_{a}, \mathbf{e}_{a}\right)$ we associate a pair $\left(\mathbf{x}_{b}, \mathbf{e}_{b}\right)$ as follows

$$
\mathbf{x}_{b}:=\left(3 \sqrt{2} \sqrt{\frac{\bar{P}_{x}}{\underline{P}_{x}}}+2\right) \mathbf{x}_{a}, \quad \mathbf{e}_{b}:=6 \mathbf{e}_{a} .
$$

and the real number

$$
\rho_{0}\left(\mathbf{x}_{a}, \mathbf{e}_{a}\right):=\frac{\bar{P}_{x}}{2 \underline{P}_{x}}\left[\mathbf{f}_{x x}\left(\mathbf{x}_{a}, \mathbf{e}_{a}\right) \mathbf{x}_{a}+\mathbf{f}_{e x}\left(\mathbf{x}_{a}, \mathbf{e}_{a}\right) \mathbf{e}_{a}+\underline{P}_{x} \mathbf{f}_{x x}\left(\mathbf{x}_{b}, \mathbf{e}_{a}\right)\left(\mathbf{x}_{b}+\mathbf{x}_{a}\right)\right] .
$$

By definition, the function $\rho_{0}$ is a non-decreasing function satisfying $\rho_{0}(0,0)=0$. With the above definitions, let $\mathbf{x}_{a}, \mathbf{e}_{a}>0$ be any pair of positive numbers satisfying the following two inequalities

$$
\begin{aligned}
9 \bar{P}_{x} \mathbf{x}_{a}^{2}+9 \mathbf{e}_{a}^{2} & \leq \frac{1}{2} \min \left\{\underline{P_{x}}\left(\mathbf{x}_{b}-\mathbf{x}_{a}\right)^{2},\left(\mathbf{e}_{b}-\mathbf{e}_{a}\right)^{2}\right\}, \\
\rho_{0}\left(\mathbf{x}_{a}, \mathbf{e}_{a}\right) & \leq \frac{1}{3} \alpha \underline{P}_{x},
\end{aligned}
$$

that depends only on the triplet of numbers $\left(\bar{P}_{x}, \underline{P}_{x}, \alpha\right)$. Such $\mathbf{x}_{a}, \mathbf{e}_{a}>0$ always exists in view of the above definition of $\mathbf{x}_{b}, \mathbf{e}_{b}$ in (95) and the properties of $\rho_{0}$. Then, with $\mathbf{x}_{a}, \mathbf{e}_{a}$ and $\mu \geq 1$ being fixed, for any given $\boldsymbol{\zeta}_{a}>0$, let $h>0$ a small positive number satisfying the following inequality

$$
h \leq \frac{1}{3} \min \left\{\frac{1}{\left(3 \zeta_{a}\right)^{2}} \min \left\{\underline{P_{x}}\left(\mathbf{x}_{b}-\mathbf{x}_{a}\right)^{2},\left(\mathbf{e}_{b}-\mathbf{e}_{a}\right)^{2}\right\}, \mu \frac{\alpha \underline{P}_{x}}{\mathbf{q}_{x}\left(\mathbf{x}_{b}, \mathbf{e}_{b}\right)^{2}}\right\}
$$

Finally, with $\mathbf{x}_{a}, \mathbf{e}_{a}, \mathbf{x}_{b}, \mathbf{e}_{b}, \mu$ and $h$ fixed, we select $\sigma_{a}^{\star}$ as

$$
\sigma_{a}^{\star}:=\frac{\left[\bar{P}_{x} \mathbf{f}_{e}\left(\mathbf{x}_{b}, \mathbf{e}_{b}\right)+\mathbf{q}_{x}\left(\mathbf{x}_{b}, \mathbf{e}_{b}\right)\right]^{2}}{2\left[\alpha \underline{P}_{x}-\rho_{0}\left(\mathbf{x}_{a}, \mathbf{e}_{a}\right)-\frac{h}{\mu} \mathbf{q}_{x}\left(\mathbf{x}_{b}, \mathbf{e}_{b}\right)^{2}\right]}+\frac{\mu}{2 h}+\mathbf{q}_{e}\left(\mathbf{x}_{b}, \mathbf{e}_{b}\right)+\frac{h}{\mu} \mathbf{q}_{e}\left(\mathbf{x}_{b}, \mathbf{e}_{b}\right)^{2} .
$$

Again, $n_{o}$ plays no role in the above inequalities. This implies the triple $\left(\mathbf{x}_{a}, \mathbf{e}_{a}, \sigma_{1}^{\star}\right)$ does not depend on $n_{o}$.

With (88), $f \in \mathscr{F}\left(\mathbf{x}_{b}, \mathbf{e}_{b}, \bar{P}_{x}, \underline{P}_{x}, \alpha\right)$ and $q \in \mathscr{Q}\left(\mathbf{x}_{b}, \mathbf{e}_{b}\right)$, and by using inequality (63), we obtain

$$
\begin{aligned}
|\tilde{q}(t, \tilde{x}, \tilde{e})| & \leq \mathbf{q}_{e}\left(\mathbf{x}_{b}, \mathbf{e}_{b}\right)|\tilde{e}|+\mathbf{q}_{x}\left(\mathbf{x}_{b}, \mathbf{e}_{b}\right)|\tilde{x}| \\
|\delta \tilde{f}(t, \tilde{x}, \tilde{e})| & \leq \mathbf{f}_{e}\left(\mathbf{x}_{b}, \mathbf{e}_{b}\right)|\tilde{e}|+\mathbf{f}_{x x}\left(\mathbf{x}_{b}, \mathbf{e}_{a}\right)|\tilde{x}|^{2}
\end{aligned}
$$

for all $(x, e) \in \mathscr{S}\left(\mathbf{x}_{b}, \mathbf{e}_{b}\right), t \in[0, T]$. Also, when $F_{p}$ is sufficiently close to $F(t)$, we can still use the function $P_{x}$ defined in (4), (5) to build a candidate Lyapunov function for the $\tilde{x}$-subsystem. For this, let us define the function

$$
\delta F(t):=F_{p}(t)-F(t)=\frac{\partial f}{\partial x}\left(t, x_{p}(t), e_{p}(t)\right)-\frac{\partial f}{\partial x}(t, 0,0) .
$$

It is $T$-periodic and satisfies

$$
|\delta F(t)| \leq\left|F_{p}(t)-F(t)\right| \leq \mathbf{f}_{x x}\left(\mathbf{x}_{a}, \mathbf{e}_{a}\right) \mathbf{x}_{a}+\mathbf{f}_{e x}\left(\mathbf{x}_{a}, \mathbf{e}_{a}\right) \mathbf{e}_{a},
$$


for all $t \in[0, T]$. Therefore, by using (5), we compute

$$
\begin{aligned}
\dot{P}_{x}(t)+P_{x}(t) F_{p}(t)+F_{p}(t)^{\top} P_{x}(t) & \leq-2 \alpha P_{x}+\left[P_{x}(t) \delta F(t)+\delta F(t)^{\top} P_{x}(t)\right] \\
& \leq-2\left(\alpha \underline{P}_{x}-\frac{\bar{P}_{x}}{\underline{P}_{x}}\left[\mathbf{f}_{x x}\left(\mathbf{x}_{a}, \mathbf{e}_{a}\right) \mathbf{x}_{a}+\mathbf{f}_{e x}\left(\mathbf{x}_{a}, \mathbf{e}_{a}\right) \mathbf{e}_{a}\right]\right) I
\end{aligned}
$$

for all $(x, e) \in \mathscr{S}\left(\mathbf{x}_{b}, \mathbf{e}_{b}\right), t \in[0, T]$. Hence, by defining $V_{x}:=\tilde{x}^{\top} P_{x} \tilde{x}$, we obtain

$$
\begin{aligned}
\dot{V}_{x} & =2 \tilde{x}^{\top} P_{x}(t)\left(F_{p}(t) \tilde{x}+\delta \tilde{f}(t, \tilde{x}, \tilde{e})\right)+\tilde{x}^{\top} \dot{P}_{x}(t) \tilde{x} \\
& \leq-2\left(\alpha \underline{P}_{x}-\frac{\bar{P}_{x}}{\underline{P}_{x}}\left[\mathbf{f}_{x x}\left(\mathbf{x}_{a}, \mathbf{e}_{a}\right) \mathbf{x}_{a}+\mathbf{f}_{e x}\left(\mathbf{x}_{a}, \mathbf{e}_{a}\right) \mathbf{e}_{a}\right]\right)|\tilde{x}|^{2}+2 \bar{P}_{x} \mathbf{f}_{e}\left(\mathbf{x}_{b}, \mathbf{e}_{b}\right)|\tilde{e}||\tilde{x}|+2 \bar{P}_{x} \mathbf{f}_{x x}\left(\mathbf{x}_{b}, \mathbf{e}_{a}\right)|\tilde{x}|^{3} \\
& \leq-2\left[\alpha \underline{P}_{x}-\rho_{0}\left(\mathbf{x}_{a}, \mathbf{e}_{a}\right)\right]|\tilde{x}|^{2}+2 \bar{P}_{x} \mathbf{f}_{e}\left(\mathbf{x}_{b}, \mathbf{e}_{b}\right)|\tilde{e}||\tilde{x}|
\end{aligned}
$$

for all $(x, e) \in \mathscr{S}\left(\mathbf{x}_{b}, \mathbf{e}_{b}\right), t \in[0, T]$, where in the last step we used the inequality $|\tilde{x}| \leq\left|x_{p}(t)\right|+|x| \leq \mathbf{x}_{a}+\mathbf{x}_{b}$, and $\rho_{0}$ has been defined in (96). Next, the derivative of $V_{e}:=\tilde{e}^{2}$ satisfies

$$
\dot{V}_{e} \leq-2 \sigma|\tilde{e}|^{2}+2 \mu M^{\top} N_{z} \tilde{\zeta} \tilde{e}+2 \mathbf{q}_{e}\left(\mathbf{x}_{b}, \mathbf{e}_{b}\right)|\tilde{e}|^{2}+2 \mathbf{q}_{x}\left(\mathbf{x}_{b}, \mathbf{e}_{b}\right)|\tilde{x}||\tilde{e}|
$$

for all $(x, e) \in \mathscr{S}\left(\mathbf{x}_{b}, \mathbf{e}_{b}\right), t \in[0, T]$, in which we used (100). Finally, for the $\tilde{\zeta}$ dynamics, define the function $V_{\zeta}=\tilde{\zeta}^{\top} N_{z} \tilde{\zeta}$. By using again inequality (100), its derivative satisfies

$$
\begin{aligned}
\dot{V}_{\zeta} & =2 \zeta^{\top} N_{z}\left[\left(\Phi-\mu M M^{\top} N_{z}\right) \tilde{\zeta}-M \tilde{q}(t, \tilde{x}, \tilde{e})\right] \\
& \leq-\mu\left|M^{\top} N_{z} \tilde{\zeta}\right|^{2}+\frac{2}{\mu} \mathbf{q}_{e}\left(\mathbf{x}_{b}, \mathbf{e}_{b}\right)^{2}|\tilde{e}|^{2}+\frac{2}{\mu} \mathbf{q}_{x}\left(\mathbf{x}_{b}, \mathbf{e}_{b}\right)^{2}|\tilde{x}|^{2}
\end{aligned}
$$

for all $(x, e) \in \mathscr{S}\left(\mathbf{x}_{b}, \mathbf{e}_{b}\right), t \in[0, T]$. As a consequence, by collecting all the inequalities together, we conclude that the time derivative of the function

$$
U:=V_{x}+V_{e}+h V_{\zeta}, \quad \tilde{\chi}:=\left(|\tilde{x}|,|\tilde{e}|,\left|M^{\top} N_{z} \tilde{\zeta}\right|\right)^{\top},
$$

satisfies

$$
\dot{U} \leq-\tilde{\chi}^{\top} \mathscr{R}\left(\mathbf{x}_{a}, \mathbf{e}_{a}, \sigma, \mu, h\right) \tilde{\chi},
$$

with $\mathscr{R}$ defined as

$$
\mathscr{R}\left(\mathbf{x}_{a}, \mathbf{e}_{a}, \sigma, \mu, h\right):=\left(\begin{array}{ccc}
2\left(\alpha \underline{P}_{x}-\rho_{11}\left(\mathbf{x}_{a}, \mathbf{e}_{a}, \mu, h\right)\right) & -\rho_{12}\left(\mathbf{x}_{a}, \mathbf{e}_{a}\right) & 0 \\
-\rho_{12}\left(\mathbf{x}_{a}, \mathbf{e}_{a}\right) & 2\left(\sigma-\rho_{22}\left(\mathbf{x}_{a}, \mathbf{e}_{a}, \mu, h\right)\right) & -\mu \\
0 & -\mu & \mu h
\end{array}\right),
$$

and where the functions $\rho_{11}, \rho_{12}$ and $\rho_{22}$ are non-decreasing functions defined as

$$
\begin{aligned}
\rho_{11}\left(\mathbf{x}_{a}, \mathbf{e}_{a}, \mu, h\right) & :=\rho_{0}\left(\mathbf{x}_{a}, \mathbf{e}_{a}\right)+\frac{h}{\mu} \mathbf{q}_{x}\left(\mathbf{x}_{b}, \mathbf{e}_{b}\right)^{2}, \\
\rho_{12}\left(\mathbf{x}_{a}, \mathbf{e}_{a}\right) & :=\bar{P}_{x} \mathbf{f}_{e}\left(\mathbf{x}_{b}, \mathbf{e}_{b}\right)+\mathbf{q}_{x}\left(\mathbf{x}_{b}, \mathbf{e}_{b}\right), \\
\rho_{22}\left(\mathbf{x}_{a}, \mathbf{e}_{a}, \mu, h\right) & :=\mathbf{q}_{e}\left(\mathbf{x}_{b}, \mathbf{e}_{b}\right)+\frac{h}{\mu} \mathbf{q}_{e}\left(\mathbf{x}_{b}, \mathbf{e}_{b}\right)^{2} .
\end{aligned}
$$

According to Sylvester's criterion, the matrix $\mathscr{R}$ is positive definite if and only if the leading principal minors are all strictly positive, that is, we need to satisfy

$$
\begin{array}{r}
\alpha \underline{P}_{x}-\rho_{11}>0, \\
4\left(\alpha \underline{P}_{x}-\rho_{11}\right)\left(\sigma-\rho_{22}\right)-\rho_{12}^{2}>0, \\
2\left(\alpha \underline{P}_{x}-\rho_{11}\right)\left[2\left(\sigma-\rho_{22}\right) h-\mu\right]-h \rho_{12}^{2}>0,
\end{array}
$$

where the arguments of the functions $\rho_{i j}$ have been omitted for compactness. These inequalities are always satisfied for any $\sigma>\sigma_{a}^{\star}$ since

- (105) is implied by (97b) and (98).

- (106) is implied by (105) and (107).

- (107) is implied by (99). 
As a consequence, for any $\sigma>\sigma_{a}^{\star}$, there exists a positive real number $v>0$, which depends only on $\left(\mathbf{x}_{a}, \mathbf{e}_{a}, \sigma, \mu, h\right)$, so that the derivative of $U$ satisfies the following inequality

$$
\dot{U} \leq-v\left(|\tilde{x}|^{2}+|\tilde{e}|+\left|M^{\top} N_{z} \tilde{\zeta}\right|^{2}\right)
$$

for all $(x, e) \in \mathscr{S}\left(\mathbf{x}_{b}, \mathbf{e}_{b}\right), t \in[0, T]$.

Next, to make the right hand side of (108) negative definite, consider the function

$$
V_{\zeta, s}:=\tilde{\zeta}^{\top}\left(N_{z}+\kappa P_{\zeta}\right) \tilde{\zeta}
$$

where $\kappa P_{\zeta}$ is defined in Lemma 1 and depends on $n_{o}$. By using the inequality (31), we compute its derivative. It satisfies

$$
\begin{aligned}
\dot{V}_{\zeta, s} & \leq-\mu\left|M^{\top} N_{z} \tilde{\zeta}\right|^{2}-\kappa \tilde{\zeta}^{\top} N_{z} \tilde{\zeta}-2\left[\tilde{\zeta}^{\top} N_{z} M\right] \tilde{q}(t, \tilde{x}, \tilde{e})-2 \kappa\left[\tilde{\zeta}^{\top} N_{z}^{1 / 2}\right]\left[N_{z}^{-1 / 2} P_{\zeta} M\right] \tilde{q}(t, \tilde{x}, \tilde{e}) \\
& \leq-\frac{\kappa}{2} \tilde{\zeta}^{\top} N_{z} \tilde{\zeta}+\frac{1}{\mu} q(t, \tilde{x}, \tilde{e})^{2}+2 \kappa\left|N_{z}^{-1 / 2} P_{\zeta} M\right|^{2} q(t, \tilde{x}, \tilde{e})^{2} \\
& \leq-\frac{\kappa}{2} \tilde{\zeta}^{\top} N_{z} \tilde{\zeta}+2\left[\frac{1}{\mu}+2 \kappa\left|N_{z}^{-1 / 2} P_{\zeta} M\right|^{2}\right]\left[\mathbf{q}_{e}\left(\mathbf{x}_{b}, \mathbf{e}_{b}\right)^{2}|\tilde{e}|^{2}+\mathbf{q}_{x}\left(\mathbf{x}_{b}, \mathbf{e}_{b}\right)^{2}|\tilde{x}|^{2}\right],
\end{aligned}
$$

for all $(x, e) \in \mathscr{S}\left(\mathbf{x}_{b}, \mathbf{e}_{b}\right), t \in[0, T]$. Hence, by letting

$$
c:=\frac{v}{2\left[\frac{1}{\mu}+2 \kappa\left|N_{z}^{-1 / 2} P_{\zeta} M\right|^{2}\right] \max \left\{\mathbf{q}_{e}\left(\mathbf{x}_{b}, \mathbf{e}_{b}\right)^{2}, \mathbf{q}_{x}\left(\mathbf{x}_{b}, \mathbf{e}_{b}\right)^{2}\right\}}
$$

we conclude, by using (108), that the derivative of $U_{s}:=U+c V_{\zeta, s}$ satisfies

$$
\dot{U}_{s} \leq-\frac{v}{2}\left(|\tilde{x}|^{2}+|\tilde{e}|^{2}+\left|M^{\top} N_{z} \tilde{\zeta}\right|^{2}\right)-\frac{c \kappa}{2} \tilde{\zeta}^{\top} N_{z} \tilde{\zeta}
$$

for all $(x, e) \in \mathscr{S}\left(\mathbf{x}_{b}, \mathbf{e}_{b}\right), t \in[0, T]$. At this point is important to note for the exponential stability that, on the left hand side we have the time derivative of a function which, although time dependent via its $V_{x}$ component, can be upperbounded by time independent positive definite quadratic forms of $(\tilde{x}, e, \tilde{\zeta})$ and on the right hand side, we have a time independent positive definite quadratic form.

Now, as a consequence of inequalities (97a) and (98), we also have

$$
9 \bar{P}_{x} \mathbf{x}_{a}^{2}+9 \mathbf{e}_{a}^{2}+9 h \zeta_{a}^{2} \leq \frac{5}{6} \min \left\{\underline{P_{x}}\left(\mathbf{x}_{b}-\mathbf{x}_{a}\right)^{2},\left(\mathbf{e}_{b}-\mathbf{e}_{a}\right)^{2}\right\} .
$$

Then, with the definitions of $\mathbf{x}_{b}$ and $\mathbf{e}_{b}$ given in (95), let

$$
\begin{aligned}
\mathscr{O} & :=\left\{(\tilde{x}, \tilde{e}, \tilde{\zeta}):|\tilde{x}|<\mathbf{x}_{b}-\mathbf{x}_{a},|\tilde{e}|<\mathbf{e}_{b}-\mathbf{e}_{a}\right\} \\
& =\left\{(\tilde{x}, \tilde{e}, \tilde{\zeta}):|\tilde{x}|<\left(1+3 \sqrt{2} \sqrt{\frac{\bar{P}_{x}}{\underline{P}_{x}}}\right) \mathbf{x}_{a},|\tilde{e}|<5 \mathbf{e}_{b}\right\} .
\end{aligned}
$$

Our interest in this set is coming from the following implication, given by (88),

$$
(\tilde{x}, \tilde{e}, \tilde{\zeta}) \in \mathscr{O} \Longrightarrow\left\{\begin{array}{l}
|x| \leq|\tilde{x}|+\left|x_{p}\right|<\mathbf{x}_{b} \\
|e| \leq|\tilde{e}|+\left|e_{p}\right|<\mathbf{e}_{b}
\end{array}\right.
$$

As a consequence, let $(\tilde{x}(t), \tilde{e}(t), \tilde{\zeta}(t))$ be an arbitrary solution with $[0, \tau)$ as right maximal interval of definition into the set $\mathscr{O}$ and with initial conditions in the set $\mathscr{N}_{\zeta}$ defined in (89), that is,

$$
N_{\zeta}=\left\{(x, e, \zeta):\left|x-x_{p}(0)\right| \leq 3 \mathbf{x}_{a},\left|e-e_{p}(0)\right| \leq 3 \mathbf{e}_{a}, \sqrt{\left(\zeta-\zeta_{p}(0)\right)^{\top} N_{z}\left(\zeta-\zeta_{p}(0)\right)} \leq 3 \zeta_{a}\right\}
$$

Note that by using the definitions of $\tilde{x}, \tilde{e}, \tilde{\zeta}$ and the bounds on the periodic solution $\left(x_{p}, e_{p}, \zeta_{p}\right)$ in (88), $\mathscr{N}_{z}$ is a subset of $\left\{(\tilde{x}, \tilde{e}, \tilde{\zeta}):|\tilde{x}| \leq 4 \mathbf{x}_{a},|\tilde{e}| \leq 4 \mathbf{e}_{a}\right\}$ and therefore, by using the definitions of $\mathbf{x}_{b}$, and $\mathbf{e}_{b}$ given in (95), $\mathscr{N}_{z}$ is a subset of $\mathscr{O}$. 
Now, if $\tau$ is finite, we have

$$
\lim _{t \rightarrow \tau}|\tilde{x}(t)|=\mathbf{x}_{b}-\mathbf{x}_{a}, \quad \text { or } \quad \lim _{t \rightarrow \tau}|\tilde{e}(t)|=\mathbf{e}_{b}-\mathbf{e}_{a}, \quad \text { or } \quad \lim _{t \rightarrow \tau} \tilde{\zeta}(t)^{\top} N_{z} \tilde{\zeta}(t)=\infty .
$$

But because of (111), we can use (108) and (89) to get

$$
U(t) \leq U(0) \leq \bar{P}_{x}\left(3 \mathbf{x}_{a}\right)^{2}+\left(3 \mathbf{e}_{a}\right)^{2}+h\left(3 \boldsymbol{\zeta}_{a}\right)^{2}
$$

for all $t \in[0, \tau)$, and in particular, by using the definition of $U$ and inequality (110),

$$
\begin{aligned}
|\tilde{x}(t)| & \leq \sqrt{\frac{1}{\underline{P}_{x}} U(t)}<\mathbf{x}_{b}-\mathbf{x}_{a} \\
|\tilde{e}(t)| & \leq \mathbf{e}_{b}-\mathbf{e}_{a} \\
\tilde{\zeta}(t)^{\top} N_{z} \tilde{\zeta}(t) & \leq \frac{1}{h} \min \left\{\underline{P_{x}}\left(\mathbf{x}_{b}-\mathbf{x}_{a}\right)^{2},\left(\mathbf{e}_{p}-\mathbf{e}_{a}\right)^{2}\right\}
\end{aligned}
$$

for all $t \in[0, \tau)$. This contradicts the consequences of having $\tau$ finite. So $\tau$ is infinite and with the inequality (109) and the remark following it, we conclude the periodic solution is asymptotically stable and locally exponentially stable. Its domain of attraction contains the set $\mathscr{N}_{\zeta}$, defined in (89). This concludes the proof.

\section{A.5 Proof of Item 1) of Theorem 1}

To establish the result of item 1 of Theorem 1, we rely on Propositions 1 and 2 . We start by defining the numbers $\mathbf{x}, \mathbf{e}, \mathbf{z}$, and $\sigma^{\star}$ of the claim. Let $\mathbf{x}_{a}, \mathbf{e}_{a}$ be given by Proposition 2 . Then let $\mathbf{x}_{p}, \mathbf{e}_{p}, \mathbf{r}_{x}, \mathbf{r}_{e}, \boldsymbol{\zeta}_{p}, \sigma_{p}^{\star}$ be given by Proposition 1, which thanks to Remark 2, can be assumed to satisfy

$$
\mathbf{x}_{p} \leq \mathbf{x}_{a} \quad, \quad \mathbf{e}_{p} \leq \mathbf{e}_{a} .
$$

We define

and

$$
\mathbf{x}:=\mathbf{x}_{p}, \quad \mathbf{e}:=\mathbf{e}_{p},
$$

$$
\mathbf{z}:=\zeta_{p}+\bar{N}_{z} \mathbf{e}
$$

with $\bar{N}_{z}$ defined in (7a). We choose $\mu \geq 1$ arbitrary. Then, with

$$
\zeta_{a}:=\zeta_{p}+2 \bar{N}_{z} \mathbf{e}
$$

Proposition 2 gives us $\sigma_{a}^{\star}$. We select

$$
\sigma^{\star}:=\max \left\{\sigma_{p}^{\star}, \sigma_{a}^{\star}\right\}
$$

With these numbers defined, we consider system (1), (9) for some given $\sigma>\sigma^{\star}$, and some given $n_{o}$. We apply the change of coordinates (27) to the closed-loop system (1), (9) to obtain (28). Proposition 1 guarantees the existence of a periodic solution $\left(x_{p}, e_{p}, \zeta_{p}\right)$ to system (28) and therefore a periodic solution $\left(x_{p}, e_{p}, z_{p}\right)$ to (1)-(9), with

With (10) and (7a), it satisfies

$$
z_{p}=\zeta_{p}+M e_{p}
$$

$$
\begin{aligned}
\sup _{t \in[0, T]}\left|x_{p}(t)\right| & \leq \min \left\{\mathbf{x}_{p}, \frac{\mathbf{r}_{x}}{\sqrt{\sigma}}\right\} \leq \mathbf{x}, \\
\sup _{t \in[0, T]}\left|e_{p}(t)\right| & \leq \min \left\{\mathbf{e}, \frac{\mathbf{r}_{e}}{\sqrt{\sigma}}\right\} \leq \mathbf{e}, \\
\sup _{t \in[0, T]} \sqrt{\zeta_{p}(t)^{\top} N_{z} \zeta_{p}(t)} & \leq \zeta_{p}, \\
\sup _{t \in[0, T]} \sqrt{z_{p}(t)^{\top} N_{z} z_{p}(t)} & \leq \sup _{t \in[0, T]} \sqrt{\left(\zeta_{p}(t)+M e_{p}(t)\right)^{\top} N_{z}\left(\zeta_{p}(t)+M e_{p}(t)\right.} \\
& \leq \sup _{t \in[0, T]} \sqrt{\zeta_{p}(t)^{\top} N_{z} \zeta_{p}(t)}+\sqrt{M^{\top} N_{z} M} \sup _{t \in[0, T]}\left|e_{p}(t)\right| \\
& \leq \zeta_{p}+\bar{N}_{z} \mathbf{e}_{p}=\mathbf{z}
\end{aligned}
$$


This establishes the inequalities (12) and (14) with $\psi_{1}:=\mathbf{r}_{e}$.

Moreover Proposition 2 guarantees the periodic solution $\left(x_{p}, e_{p}, \zeta_{p}\right)$ is locally exponentially stable with a domain of attraction that includes the set $\mathscr{N}_{\zeta}\left(\mathbf{x}_{a}, \mathbf{e}_{a}, \boldsymbol{\zeta}_{a}\right)$ defined in (89). Hence the domain of attraction of $\left(x_{p}, e_{p}, z_{p}\right)$ contains the set

$\left\{(x, e, z):\left|x-x_{p}(0)\right| \leq 3 \mathbf{x}_{a}, \quad\left|e-e_{p}(0)\right| \leq 3 \mathbf{e}_{a}\right.$,

$$
\left.\sqrt{\left(z-z_{p}(0)-M\left(e-e_{p}(0)\right)^{\top} N_{z}\left(z-z_{p}(0)-M\left(e-e_{p}(0)\right)\right.\right.} \leq 3 \zeta_{a}\right\} .
$$

But we have the implications

$$
\begin{aligned}
&\left\{|x| \leq 2 \mathbf{x} \quad \& \quad\left|x_{p}(0)\right| \leq \mathbf{x}\right\} \Rightarrow\left|x-x_{p}(0)\right| \leq|x|+\left|x_{p}(0)\right| \leq 3 \mathbf{x} \\
&\left\{|e| \leq 2 \mathbf{e} \& \quad\left|e_{p}(0)\right| \leq \mathbf{e}\right\} \Rightarrow \quad\left|e-e_{p}(0)\right| \leq|e|+\left|e_{p}(0)\right| \leq 3 \mathbf{e} \\
&\left\{\sqrt{z^{\top} N_{z} z} \leq 2 \mathbf{z} \& \quad \sqrt{z_{p}(0)^{\top} N_{z} z_{p}(0)} \leq \mathbf{z} \quad \& \quad|e| \leq 2 \mathbf{e} \quad \& \quad\left|e_{p}(0)\right| \leq \mathbf{e}\right\} \\
& \Rightarrow \sqrt{\left(z-z_{p}(0)-M\left(e-e_{p}(0)\right)^{\top} N_{z}\left(z-z_{p}(0)-M\left(e-e_{p}(0)\right)\right.\right.} \\
& \leq \sqrt{\left(z-z_{p}(0)\right)^{\top} N_{z}\left(z-z_{p}(0)\right)}+\sqrt{M^{\top} N_{z} M}\left|e-e_{p}(0)\right| \\
& \leq \sqrt{z^{\top} N_{z} z}+\sqrt{z_{p}(0)^{\top} N_{z} z_{p}(0)}+\bar{N}_{z} 3 \mathbf{e} \\
& \leq 3\left(\mathbf{z}+\bar{N}_{z} \mathbf{e}\right)=3\left(\boldsymbol{\zeta}_{p}+2 \bar{N}_{z} \mathbf{e}\right) \leq 3 \boldsymbol{\zeta}_{a}
\end{aligned}
$$

Hence the set $\mathscr{N}(\mathbf{x}, \mathbf{e}, \mathbf{z})$ defined in (13) is contained in the above set and therefore in the domain of attraction of $\left(x_{p}, e_{p}, z_{p}\right)$.

\section{A.6 Proof of Items 2)-4) of Theorem 1}

First, note that item 2) of Theorem 1 is a straightforward consequence of the inequalities (46b) and (46a) claimed in the statement of Proposition 1, that is $\psi_{1}:=\mathbf{r}_{e}$ and $\psi_{x}:=\mathbf{r}_{x}$.

Then, the $C^{2}$ periodic solution $\left(x_{p}, e_{p}, \zeta_{p}\right)$ given by Item 1$)$ satisfies (see (28))

$$
\begin{aligned}
& \dot{e}_{p}=-\sigma e_{p}+\mu M^{\top} N_{z} \zeta_{p}+q\left(t, x_{p}, e_{p}\right), \\
& \dot{\zeta}_{p}=\left(\Phi-\mu M M^{\top} N_{z}\right) \zeta_{p}-M q\left(t, x_{p}, e_{p}\right)
\end{aligned}
$$

and it is the sum of its Fourier series, i.e.

$$
\begin{aligned}
e_{p}(t) & =\sum_{k \in \mathbb{Z}} e_{p k} \exp \left(\mathrm{i} k \frac{2 \pi}{T} t\right), \\
\zeta_{p}(t) & =\sum_{k \in \mathbb{Z}} \zeta_{p k} \exp \left(\mathrm{i} k \frac{2 \pi}{T} t\right), \\
q\left(t, x_{p}(t), e_{p}(t)\right) & =\sum_{k \in \mathbb{Z}} q_{p k} \exp \left(\mathrm{i} k \frac{2 \pi}{T} t\right),
\end{aligned}
$$

where the index $k$ in $\{0, \ldots, \infty\}$ denotes the $k$-th Fourier coefficient. Because of (117), $e_{p k}, \zeta_{p k}$ satisfy

$$
\begin{aligned}
\left(k i \frac{2 \pi}{T}+\sigma\right) e_{p k} & =\mu M^{\top} N_{z} \zeta_{p k}+q_{p k} \\
\left(k i \frac{2 \pi}{T}-\Phi+\mu M M^{\top} N_{z}\right) \zeta_{p k} & =-M q_{p k}
\end{aligned}
$$

When we are interested in expressing the $\zeta_{p}$ dynamics oscillator by oscillator, it is appropriate to exhibit the corresponding components of $\zeta_{p k}$ as

$$
\zeta_{p k}=\left(\zeta_{p k 0}, \ldots, \zeta_{p k \ell}, \ldots \zeta_{p k n_{o}}\right),
$$


so where the index $\ell$, or $m$ below, in $\left\{0, \ldots, n_{o}\right\}$ refers to the $\ell$-th component of $\zeta_{p k}$. With (10), (118) becomes

$$
\begin{aligned}
\left(k i \frac{2 \pi}{T}+\sigma\right) e_{p k} & =\mu \sum_{m=0}^{n_{o}} M_{m}^{\top} N_{z m} \zeta_{p k m}+q_{p k}, \\
\left(\begin{array}{cc}
k i \frac{2 \pi}{T} & \omega_{\ell} \\
-\omega_{\ell} & k i \frac{2 \pi}{T}
\end{array}\right) \zeta_{p k \ell}+\mu M_{\ell} \sum_{m=0}^{n_{o}} M_{m}^{\top} N_{z m} \zeta_{p k m} & =-M_{\ell} q_{p k} \quad \forall \ell \in\left\{0,1, \ldots, n_{o}\right\} .
\end{aligned}
$$

Assume that for some $\ell$ in $\left\{0,1, \ldots, n_{o}\right\}$, we have

$$
\omega_{\ell}=\mathfrak{K}_{\ell} \frac{2 \pi}{T}
$$

where $\mathfrak{K}_{\ell}$ is an integer. With (119), this implies in particular

$$
\mathfrak{K}_{\ell} \frac{2 \pi}{T}\left(\begin{array}{cc}
\mathrm{i} & 1 \\
-1 & \mathrm{i}
\end{array}\right) \zeta_{p \mathfrak{K}_{\ell} \ell}=-M_{\ell}\left[\mu \sum_{m=0}^{n_{o}} M_{m}^{\top} N_{z m} \zeta_{p \mathfrak{K}_{\ell} m}+q_{p \mathfrak{K}_{\ell}}\right]=-M_{\ell}\left(k \mathrm{i} \frac{2 \pi}{T}+\sigma\right) e_{p \mathfrak{K}_{\ell}}
$$

Then, with the identities

$$
(\mathrm{i}-1)\left(\begin{array}{cc}
\mathrm{i} & 1 \\
-1 & \mathrm{i}
\end{array}\right)=0 \quad, \quad(\mathrm{i}-1) M_{\ell}=\left(\begin{array}{l}
\mathrm{i} \\
0
\end{array}\right),
$$

we obtain finally

$$
0=e_{p \mathfrak{K}_{\ell}}=\int_{0}^{T} \cos \left(\mathfrak{K}_{\ell} \frac{2 \pi}{T} t\right) e_{p}(t)+\mathrm{i} \int_{0}^{T} \sin \left(\mathfrak{K}_{\ell} \frac{2 \pi}{T} t\right) e_{p}(t) .
$$

This is (15) in Item 3)

To establish Item 4), we note that (118) gives

$$
\zeta_{p k}=-\left(k \mathrm{i} \frac{2 \pi}{T}-\Phi+\mu M M^{\top} N_{z}\right)^{-1} M q_{p k}=-\frac{\left(k \mathrm{i} \frac{2 \pi}{T} I-\Phi\right)^{-1} M}{1+\mu M^{\top} N_{z}\left(k \mathrm{i} \frac{2 \pi}{T} I-\Phi\right)^{-1} M} q_{p k},
$$

and therefore

$$
e_{p k}=\left(q_{p k}+\mu M^{\top} N_{z} \zeta_{p k}\right) \frac{1}{k i \frac{2 \pi}{T}+\sigma}=\frac{1}{1+\mu M^{\top} N_{z}\left(k \mathrm{i} \frac{2 \pi}{T} I-\Phi\right)^{-1} M} \frac{1}{k \mathrm{i} \frac{2 \pi}{T}+\sigma} q_{p k} .
$$

With the definitions $(10)$, where $\omega_{0}=0$, we have

$$
\begin{aligned}
M^{\top} N_{z}\left(k \mathrm{i} \frac{2 \pi}{T} I-\Phi\right)^{-1} M & =\frac{n_{z 0}}{k \mathrm{i} \frac{2 \pi}{T}}+\sum_{\ell=1}^{n_{o}}\left(\begin{array}{ll}
1 & 0
\end{array}\right) n_{z \ell}\left(\begin{array}{cc}
k \mathrm{i} \frac{2 \pi}{T} & \omega_{\ell} \\
-\omega_{\ell} & k \mathrm{i} \frac{2 \pi}{T}
\end{array}\right)\left(\begin{array}{l}
1 \\
0
\end{array}\right) \\
& =\mathrm{i} k \frac{2 \pi}{T} \sum_{\ell=0}^{n_{o}} \frac{n_{z \ell}}{\omega_{\ell}^{2}-k^{2}\left[\frac{2 \pi}{T}\right]^{2}}
\end{aligned}
$$

and therefore,

$$
\left|e_{p k}\right|^{2}=\frac{1}{1+\mu^{2}\left[k \frac{2 \pi}{T} \sum_{\ell=0}^{n_{o}} \frac{n_{z \ell}}{\omega_{\ell}^{2}-k^{2}\left[\frac{2 \pi}{T}\right]^{2}}\right]^{2}} \frac{\left|q_{p k}\right|^{2}}{k^{2}\left[\frac{2 \pi}{T}\right]^{2}+\sigma^{2}}
$$

Note that the first factor of the right hand side comes with the presence of the internal model. Indeed, without this model, we would get

$$
\left|e_{p k}\right|^{2}=\frac{\left|q_{p k}\right|^{2}}{k^{2}\left[\frac{2 \pi}{T}\right]^{2}+\sigma^{2}} .
$$

So now, if we suppose that all $\omega_{\ell}$ in (9) are selected as

$$
\omega_{\ell}=\ell \frac{2 \pi}{T}, \quad \forall \ell \in\left\{1, \ldots, n_{o}\right\}
$$


then, from (120), we obtain

$$
\begin{aligned}
\left|e_{p k}\right|^{2} & =0 & & \forall|k| \leq n_{o}, \\
& \leq \frac{1}{\left(n_{o}+1\right)^{2}\left[\frac{2 \pi}{T}\right]^{2}+\sigma^{2}}\left|q_{p k}\right|^{2} & & \forall|k| \geq n_{o}+1 .
\end{aligned}
$$

Parseval's theorem then gives us

$$
\begin{aligned}
\frac{1}{T} \int_{0}^{T}\left|e_{p}(t)\right|^{2} d t & =\sum_{k \in \mathbb{Z}}\left|e_{p k}\right|^{2} \\
& \leq \frac{1}{\left(n_{o}+1\right)^{2}\left[\frac{2 \pi}{T}\right]^{2}+\sigma^{2}} \sum_{k:|k| \geq n_{o}+1}\left|q_{p k}\right|^{2} \\
& \leq \frac{1}{\left(n_{o}+1\right)^{2}\left[\frac{2 \pi}{T}\right]^{2}+\sigma^{2}} \frac{1}{T} \int_{0}^{T}\left|q\left(t, x_{p}(t), e_{p}(t)\right)\right|^{2} d t \\
& \leq \frac{1}{\left(n_{o}+1\right)^{2}} \frac{\mathbf{q}\left(\mathbf{x}_{p}, \mathbf{e}_{p}\right)^{2}}{\left[\frac{2 \pi}{T}\right]^{2}}
\end{aligned}
$$

This is (16), with $\psi_{2}:=\left[\frac{T}{2 \pi}\right]^{2} \mathbf{q}\left(\mathbf{x}_{p}, \mathbf{e}_{p}\right)^{2}$.

\section{B Proof of Theorem 2}

\section{B.1 Preliminaries}

A key feature of the infinite dimensional dynamical regulator (9) is that, when $e=0$, it can reproduce any desired $C^{1} T$-periodic function, as stated by the following lemma.

Lemma 5 For any $c \in \mathscr{C}_{T}^{1}(\mathbb{R})$ and any $\mu \geq 1$, there exists $z_{p} \in \mathscr{L}_{N_{z}}^{2}$ such that the solution to

$$
\dot{z}=\Phi z, \quad z(0)=z_{p}
$$

is in $\mathscr{C}_{T}^{1}\left(\mathscr{L}_{N_{z}}^{2}\right)$ and satisfies

$$
\mu M^{\top} N_{z} z(t)=c(t) \quad \forall t \geq 0
$$

with $\Phi, M, N_{z}$ defined in (17).

Proof Since $c$ is $C^{1}$ and $T$-periodic, it can be expressed as the sum of its Fourier series as

$$
c(t)=c_{0}+\sum_{k=1}^{\infty} c_{k}^{c} \cos (k \omega t)+c_{k}^{s} \sin (k \omega t), \quad \omega=\frac{2 \pi}{T} .
$$

Then, let the $k$-th component $z_{p k}$ of the vector $z_{p}$ be selected as

$$
z_{p 0}=\frac{c_{0}}{\mu}, \quad z_{p k}=\frac{1}{\mu n_{z k}} c_{k}, \quad c_{k}=\left(\begin{array}{c}
c_{k}^{c} \\
c_{k}^{s}
\end{array}\right) \quad \forall k=1, \ldots, \infty .
$$

To verify that $z_{p}$ is in the set $\mathscr{L}_{N_{z}}^{2}$ as defined in definition (19), we compute

$$
\sum_{k=1}^{\infty} n_{z k}\left|z_{p k}\right|^{2}=\frac{1}{\mu^{2}} \sum_{k=1}^{\infty} \frac{1}{n_{z k}}\left|c_{k}\right|^{2} \leq \frac{1}{\mu^{2}} \sup _{k \in \mathbb{N}_{>0}} \frac{1}{k^{2} n_{z k}} \sum_{k=1}^{\infty} k^{2}\left|c_{k}\right|^{2} .
$$

Now, $\sup _{k \in \mathbb{N}_{>0}} \frac{1}{k^{2} n_{z k}}$ is finite by assumption (7d) as well as $k\left|c_{k}\right|$ is square summable since $c$, being $C^{1}$, $\frac{d c}{d t}$ is square integrable. 
To verify (121b) holds, we remind that, component-wise, the elements of $z$ are given by the solution of the differential equations

$$
\begin{array}{ll}
\dot{z}_{0}=0, & z_{0}(0)=z_{p 0} \\
\dot{z}_{k}=\Phi_{k} z_{k}, & z_{k}(0)=z_{p k} \quad \forall k=1, \ldots, \infty,
\end{array}
$$

with $\Phi_{k}$ defined in (17). Hence

$$
\begin{aligned}
z_{0} & =z_{p 0} \\
z_{k}(t) & =\left(\begin{array}{cc}
\cos (k \omega t) & \sin (k \omega t) \\
-\sin (k \omega t) & \cos (k \omega t)
\end{array}\right) z_{p k} \quad \forall k=1, \ldots, \infty .
\end{aligned}
$$

This implies $z \in \mathscr{C}_{T}^{1}\left(\mathscr{L}_{N_{z}}^{2}\right)$, Then, by using the definition of $M, N_{z}$ in (10), we obtain

$$
\begin{aligned}
\mu M^{\top} N_{z} z(t) & =\mu z_{0}(t)+\mu \sum_{k=1}^{\infty} n_{z k} M_{k}^{\top} \exp \left(\Phi_{k} t\right) z_{p k} \\
& =\mu z_{p 0}(t)+\mu \sum_{k=1}^{\infty} n_{z k}\left[\cos (k \omega t) z_{p k}^{c}+\sin (k \omega t) z_{p k}^{s}\right] \\
& =c_{0}+\sum_{k=1}^{\infty} c_{k}^{c} \cos (k \omega t)+c_{k}^{s} \sin (k \omega t)=c(t)
\end{aligned}
$$

for all $t \geq 0$. This concludes the proof.

Now, let us define

$$
c(t):=-q(t, 0,0) .
$$

By construction $c \in \mathscr{C}_{T}^{1}([0, T] ; \mathbb{R})$. Hence, let $z_{p}$ be given with such $c$ by Lemma 5 and consider the following change of coordinates

$$
z \mapsto \zeta:=z-z_{p}(t)-M e
$$

which transforms the closed-loop system (1), (9) into

$$
\begin{aligned}
& \dot{x}=F(t) x+\delta f(t, x, e) \\
& \dot{e}=\Delta(t, x, e)-\sigma e+\mu M^{\top} N_{z} \zeta \\
& \dot{\zeta}=\left(\Phi-\mu M M^{\top} N_{z}\right) \zeta-M \Delta(t, x, e)
\end{aligned}
$$

where $F, \delta f$ are defined as in (47), namely

$$
\delta f(t, x, e):=f(t, x, e)-F(t) x, \quad F(t):=\frac{\partial f}{\partial x}(t, 0,0),
$$

and $\Delta$ is a $C^{1} T$-periodic function defined as

$$
\Delta(t, x, e):=q(t, x, e)-q(t, 0,0) .
$$

and satisfying

$$
|\Delta(t, x, e)| \leq \mathbf{q}_{e}(\mathbf{x}, \mathbf{e})|e|+\mathbf{q}_{x}(\mathbf{x}, \mathbf{e})|x|
$$

for any $(x, e) \in \mathscr{S}_{T}(\mathbf{x}, \mathbf{e})$.

The metric space $\mathscr{L}_{N_{z}}^{2}$ being complete and all functions being locally Lipschitz, we are guaranteed about existence of solutions to (125), as stated by the following known result on existence of solutions for differential equations in Banach spaces proved for example in [14, §1.1], [12, Theorem 1.8.3] or [37, Proposition VI.1.2 \& Theorem VI.3.1].

Proposition 3 Let $D$ be an arbitrary open bounded subset of $\mathbb{R}^{n} \times \mathbb{R} \times \times \mathscr{L}_{N_{z}}^{2}$, then for any $\left(x\left(t_{0}\right)\right.$, $e\left(t_{0}\right)$, $\left.\zeta\left(t_{0}\right)\right)$ in $D$ and any $t_{0}$, system (125) has a unique solution $(x(t), e(t), \zeta(t))$ with values in $D$, defined either on $\left[t_{0},+\infty\left[\right.\right.$ or only on an interval $\left[t_{0}, t_{0}+\bar{\tau}[\right.$ which is maximal in the sense that

$$
\lim _{t \rightarrow \bar{\tau}} d((x(t), e(t), \zeta(t)), \partial D)=0,
$$

where $d$ is the distance on $\mathbb{R}^{n} \times \mathbb{R} \times \mathscr{L}_{N_{z}}^{2}$ and $\partial D$ is the boundary of $D$. 


\section{B.2 Stability Analysis}

We have the following proposition

Proposition 4 Given any triplet $\left(\bar{P}_{x}, \underline{P}_{x}, \alpha\right)$ there exist strictly positive real numbers $\mathbf{x}_{\infty}, \mathbf{e}_{\infty}>0$ and, for any $\zeta_{\infty}>0$ and $\mu \geq 1$, there exists $\sigma_{\infty}^{\star}>0$ such that, the following holds.

(i) Solutions of (125) starting from the set

$$
\Omega_{\text {init }}=\left\{(x, e, \zeta) \in \mathbb{R}^{n} \times \mathbb{R} \times \mathscr{L}_{N_{z}}^{2}:|x| \leq 3 \mathbf{x}_{a},|e| \leq 3 \mathbf{e}_{a},\|\zeta\|_{N_{z}} \leq 3 \zeta_{a}\right\}
$$

are complete and bounded in positive times and $\zeta(t) \in \mathscr{L}_{N_{z}}^{2}$ for all $t \geq 0$.

(ii) The origin of (125) is Lyapunov stable with domain of attraction containing $\Omega_{\text {init- }}$.

(iii) Solutions starting from $\Omega_{\text {init }}$ satisfies

$$
\lim _{t \rightarrow \infty} x(t)=0, \quad \lim _{t \rightarrow \infty} e(t)=0 .
$$

Proof We follow similar steps of the proof of Proposition 2. First, let us define the non-decreasing function $\rho_{0}^{\infty}$ as

$$
\rho_{0}^{\infty}(\mathbf{x}):=\frac{\bar{P}_{x}}{2} \mathbf{f}_{x x}(\mathbf{x}, 0) \mathbf{x} \quad s \geq 0
$$

Let $\mathbf{x}_{b}, \mathbf{e}_{b}$ be parametrized as follows

$$
\mathbf{x}_{b}:=\left(3 \sqrt{2} \sqrt{\frac{\bar{P}_{x}}{\underline{P}_{x}}}+2\right) \mathbf{x}_{a}, \quad \mathbf{e}_{b}:=6 \mathbf{e}_{a} .
$$

and let $\mathbf{x}_{a}, \mathbf{e}_{a}$ be any pair of positive real numbers satisfying

$$
\begin{aligned}
9 \mathbf{x}_{\infty}^{2} \bar{P}_{x}+9 \mathbf{e}_{\infty}^{2} & \leq \frac{1}{2} \min \left\{\underline{P}_{x} \mathbf{x}_{b}^{2}, \mathbf{e}_{b}^{2}\right\}, \\
\rho_{0}^{\infty}\left(\mathbf{x}_{b}\right) & \leq \frac{1}{3} \alpha \underline{P}_{x}
\end{aligned}
$$

Now, given any $\zeta_{a}>0$, let $h>0$ be selected such that

$$
h \leq \frac{1}{3} \min \left\{\frac{1}{\left(3 \zeta_{a}\right)^{2}} \min \left\{\underline{P}_{x} \mathbf{x}_{b}^{2}, \mathbf{e}_{b}^{2}\right\}, \mu \frac{\alpha \underline{P}_{x}}{\mathbf{q}_{x}\left(\mathbf{x}_{b}, \mathbf{e}_{b}\right)^{2}}\right\} .
$$

Finally, let $\sigma_{\infty}^{\star}>0$ be chosen as

$$
\sigma_{\infty}^{\star}:=\frac{\left[\bar{P}_{x} \mathbf{f}_{e}\left(\mathbf{x}_{b}, \mathbf{e}_{b}\right)+\mathbf{q}_{x}\left(\mathbf{x}_{b}, \mathbf{e}_{b}\right)\right]^{2}}{2\left[\alpha \underline{P}_{x}-\rho_{0}\left(\mathbf{x}_{b}\right)-\frac{h}{\mu} \mathbf{q}_{x}\left(\mathbf{x}_{b}, \mathbf{e}_{b}\right)^{2}\right]}+\frac{\mu}{2 h}+\mathbf{q}_{e}\left(\mathbf{x}_{b}, \mathbf{e}_{b}\right)+\frac{h}{\mu} \mathbf{q}_{e}\left(\mathbf{x}_{b}, \mathbf{e}_{b}\right)^{2} .
$$

Then, by following the same steps, consider the functions

$$
V_{x}:=x^{\top} P_{x}(t) x, \quad V_{e}:=e^{2}, \quad V_{\zeta}:=\zeta^{\top} N_{z} \zeta .
$$

By using (5), and (67), their derivatives satisfy, for all $(x, e)$ in $\mathscr{S}\left(\mathbf{x}_{b}, \mathbf{e}_{b}\right), t \in[0, T]$,

$$
\begin{aligned}
\dot{V}_{x} & \leq-2 \alpha x^{\top} P_{x}(t) x+2 x^{\top} P_{x}(t) \delta f(t, x, e) \\
& \leq-2 \alpha \underline{P}_{x}|x|^{2}+2|x| \bar{P}_{x} \mathbf{f}_{e}\left(\mathbf{x}_{b}, \mathbf{e}_{b}\right)|e|+\bar{P}_{x} \mathbf{f}_{x x}\left(\mathbf{x}_{b}, 0\right)|x|^{3} \\
& \leq-2\left[\alpha \underline{P}_{x}-\rho_{0}^{\infty}\left(\mathbf{x}_{b}\right)\right]|x|^{2}+2 \bar{P}_{x} \mathbf{f}_{e}\left(\mathbf{x}_{b}, \mathbf{e}_{b}\right)|e||x|
\end{aligned}
$$

with $\rho_{0}^{\infty}$ defined as in (129), and

$$
\begin{aligned}
& \dot{V}_{e} \leq-2 \sigma|e|^{2}+2 \mu M^{\top} N_{\zeta} \zeta e+2 \mathbf{q}_{e}\left(\mathbf{x}_{b}, \mathbf{e}_{b}\right)|e|^{2}+2 \mathbf{q}_{x}\left(\mathbf{x}_{b}, \mathbf{e}_{b}\right)|x||e|, \\
& \dot{V}_{\zeta} \leq-\mu\left|M^{\top} N_{\zeta} \zeta\right|^{2}+\frac{2}{\mu} \mathbf{q}_{e}\left(\mathbf{x}_{b}, \mathbf{e}_{b}\right)^{2}|e|^{2}+\mathbf{q}_{x}\left(\mathbf{x}_{b}, \mathbf{e}_{b}\right)^{2}|x|^{2}
\end{aligned}
$$


Therefore, collecting all the inequalities together, and by defining

$$
U:=V_{x}+V_{e}+h V_{\zeta}, \quad \chi:=\left(|x|,|e|,\left|M^{\top} N_{z} \zeta\right|\right)^{\top},
$$

with $h$ selected as in (132), the time derivative of the Lyapunov function $U$ satisfies

$$
\dot{U} \leq-\chi^{\top} \mathscr{R}^{\infty}\left(\mathbf{x}_{b}, \mathbf{e}_{b}, \sigma, \mu, b\right) \chi \quad \forall(x, e, \zeta) \in \mathscr{S}_{T}\left(\mathbf{x}_{b}, \mathbf{e}_{b}\right) \times \mathscr{L}_{N_{z}}^{2},
$$

with $\mathscr{R}^{\infty}$ defined as

$$
\mathscr{R}^{\infty}\left(\mathbf{x}_{b}, \mathbf{e}_{b}, \sigma, \mu, h\right):=\left(\begin{array}{ccc}
2\left(\alpha \underline{P}_{x}-\rho_{11}^{\infty}\left(\mathbf{x}_{b}, \mathbf{e}_{b}, \mu, h\right)\right) & -\rho_{12}^{\infty}\left(\mathbf{x}_{b}, \mathbf{e}_{b}\right) & 0 \\
-\rho_{12}^{\infty}\left(\mathbf{x}_{b}, \mathbf{e}_{b}\right) & 2\left(\sigma-\rho_{22}^{\infty}\left(\mathbf{x}_{b}, \mathbf{e}_{b}, \mu, h\right)\right) & -\mu \\
0 & -\mu & \mu h
\end{array}\right),
$$

and where the functions $\rho_{11}^{\infty}, \rho_{12}^{\infty}$ and $\rho_{22}^{\infty}$ are non-decreasing functions defined as

$$
\begin{aligned}
\rho_{11}^{\infty}\left(\mathbf{x}_{b}, \mathbf{e}_{b}, \mu, h\right) & :=\rho_{0}^{\infty}\left(\mathbf{x}_{b}\right)+\frac{h}{\mu} \mathbf{q}_{x}\left(\mathbf{x}_{b}, \mathbf{e}_{b}\right)^{2}, \\
\rho_{12}^{\infty}\left(\mathbf{x}_{b}, \mathbf{e}_{b}\right) & :=\bar{P}_{x} \mathbf{f}_{e}\left(\mathbf{x}_{b}, \mathbf{e}_{b}\right)+\mathbf{q}_{x}\left(\mathbf{x}_{b}, \mathbf{e}_{b}\right), \\
\rho_{22}^{\infty}\left(\mathbf{x}_{b}, \mathbf{e}_{b}, \mu, h\right) & :=\mathbf{q}_{e}\left(\mathbf{x}_{b}, \mathbf{e}_{b}\right)+\frac{h}{\mu} \mathbf{q}_{e}\left(\mathbf{x}_{b}, \mathbf{e}_{b}\right)^{2} .
\end{aligned}
$$

According to Sylvester's criterion, the matrix $\mathscr{R}^{\infty}$ is positive definite if and only if the leading principal minors are all strictly positive, that is, we need to satisfy

$$
\begin{aligned}
\alpha \underline{P}_{x}-\rho_{11}^{\infty} & >0, \\
4\left(\alpha \underline{P}_{x}-\rho_{11}^{\infty}\right)\left(\sigma-\rho_{22}^{\infty}\right)-\left(\rho_{12}^{\infty}\right)^{2} & >0, \\
2\left(\alpha \underline{P}_{x}-\rho_{11}^{\infty}\right)\left[2\left(\sigma-\rho_{22}^{\infty}\right) h-\mu\right]-h\left(\rho_{12}^{\infty}\right)^{2} & >0,
\end{aligned}
$$

where the arguments of the functions $\rho_{i j}^{\infty}$ have been omitted for compactness. These inequalities are always satisfied for any $\sigma>\sigma_{\infty}^{\star}$ since

- (135) is implied by (131) and (132).

- (136) is implied by (135) and (137).

- (137) is implied by (133).

As a consequence, for any $\sigma>\sigma_{\infty}^{\star}$, there exists a positive real number $\varepsilon>0$ such that

$$
\dot{U} \leq-\varepsilon\left(|x|^{2}+|e|^{2}+\left|M^{\top} N_{z} \zeta\right|^{2}\right) \quad \forall(x, e, \zeta) \in \mathscr{S}\left(\mathbf{x}_{b}, \mathbf{e}_{b}\right) \times \mathscr{L}_{N_{z}}^{2} .
$$

Now, let $\bar{v}$ be the strictly positive real number defined as

$$
\bar{v}:=\min _{(x, e, \zeta) \notin \Omega_{\text {init }}}|x|^{2} \bar{P}_{x}+|e|^{2}+h \zeta^{\top} N_{z} \zeta \leq\left(3 \mathbf{x}_{a}\right)^{2} \bar{P}_{x}+\left(3 \mathbf{e}_{a}\right)^{2}+h\left(3 \boldsymbol{\zeta}_{a}\right)^{2}
$$

and define

$$
\Omega_{\max }:=\left\{(x, e, \zeta) \in \mathbb{R}^{n} \times \mathbb{R} \times \mathscr{L}_{N_{z}}^{2}: U(x, e, \zeta, t) \leq \bar{v}, t \in[0, T]\right\} .
$$

In view of the choices of $\mathbf{x}_{a}, \mathbf{x}_{b}, h$ in (131), (132), we have $\bar{v} \leq \frac{5}{6} \min \left\{\underline{P}_{x} \mathbf{x}_{b}^{2}, \mathbf{e}_{b}^{2}\right\}$ and therefore it is straightforward to see that

$$
\Omega_{\max } \subset \mathscr{S}\left(\mathbf{x}_{b}, \mathbf{e}_{b}\right) \times \mathscr{L}_{N_{z}}^{2} .
$$

Now let $D$ be an open bounded superset of $\Omega_{\max }$ contained in $\mathscr{S}\left(\mathbf{x}_{b}, \mathbf{e}_{b}\right) \times \mathscr{L}_{N_{z}}^{2}$. Let $(x(t), e(t), \zeta(t))$ be the unique solution to system (125), established by Proposition 3, with initial condition in $\Omega_{\text {init }}$ and right maximally defined on $[0, \bar{\tau}[$ on $D$. With (138), we have established

$$
U(x(t), e(t), \zeta(t), t) \leq U(x(0), e(0), \zeta(0), 0)<\bar{v} \quad \forall t \in[0, \bar{\tau}[,
$$

and therefore

$$
(x(t), e(t), \zeta(t)) \in \Omega_{\max }, \quad \zeta(t) \in \mathscr{L}_{N_{z}}^{2} \quad \forall t \in[0, \bar{\tau}[.
$$

But $\Omega_{\max }$ being a closed subset of the open set $D$, we have

$$
d\left(\Omega_{\max }, \partial D\right)>0
$$


and therefore a contradiction with (127) if $\bar{\tau}$ is finite. So the solution $(x(t), e(t), \zeta(t))$ is defined on $[0,+\infty[$ and takes it values in $\Omega_{\max }$. It follows that the function $t \mapsto\left(\left(x(t), e(t), \zeta(t), M^{\top} N_{z} \zeta(t)\right) \in \mathbb{R}^{n} \times \mathbb{R} \times \mathscr{L}_{N_{z}}^{2} \times\right.$ $\mathscr{L}_{N_{z}}^{2}$ is bounded and

$$
|q(t, x(t), e(t))| \leq \mathbf{q}(\overline{\mathbf{x}}, \overline{\mathbf{e}}) \quad \forall t \in[0,+\infty[.
$$

This shows item (i).

Next, consider the set of functions $(x, e, \zeta)$ so that $\bar{U}$ is bounded, with $\bar{U}$ defined as

$$
\bar{U}(x, e, \zeta):=|x|^{2} \bar{P}_{x}+|e|^{2}+h \zeta^{\top} N_{z} \zeta
$$

We have that $\sqrt{\bar{U}}$ defines a norm. Now, for any $\varepsilon>0$ small enough, let $\delta=\varepsilon \frac{\underline{P}_{x}}{\bar{P}_{x}}$, and consider any initial condition $(x(0), e(0), \zeta(0)) \in \Omega_{\text {init }}$ satisfying

$$
\bar{U}(x(0), e(0), \zeta(0)) \leq \delta .
$$

Using (138) along solutions, we obtain

$$
\bar{U}(t) \leq \frac{\bar{P}_{x}}{\underline{P}_{x}} U(t) \leq \frac{\bar{P}_{x}}{\underline{P}_{x}} U(0) \leq \frac{\bar{P}_{x}}{\underline{P}_{x}} \bar{U}(0) \leq \varepsilon .
$$

This shows that that the origin of (125) is Lyapunov stable with a domain of attraction which includes the set $\left\{(x, e, \zeta): \bar{U}(0) \leq \delta_{\max }\right\}$, where $\delta_{\max }>0$ is the smallest $\delta$ such that $\bar{U}\left(x^{*}, e^{*}, \zeta^{*}\right)>\delta$ implies $\left(x^{*}, e^{*}, \zeta^{*}\right) \notin \Omega_{\text {init }}$. This shows item (ii) of the theorem.

Finally, by using (138) along solutions, we obtain

$$
\lim _{\tau \rightarrow \infty} \int_{0}^{\tau} \varepsilon\left(|x(t)|^{2}+e^{2}(t)\right) d t \leq U(0)
$$

On the left hand side each term in the integrand is a function with non negative values, and, since, according to (125), the function $t \mapsto(\dot{x}(t), \dot{e}(t))$ is bounded, the function $t \mapsto(x(t), e(t)$ is Lipschitz (and therefore uniformly continuous). Hence by applying Barbalat's lemma we get

$$
\lim _{t \rightarrow \infty}|e(t)|=0, \quad \lim _{t \rightarrow \infty}|x(t)|=0,
$$

which shows item (iii) and concludes the proof.

\section{B.3 Proof of Theorem 2}

The statement of Theorem 2 follows by combining the results of Lemma 5 and Propositions 3 and 4 . To this end, let $c(t)=-q(t, 0,0)$ be given as in (123) and apply Lemma 5 to obtain the initial condition $z_{p} \in \mathscr{L}_{N_{z}}^{2}$. By further using (122) and the properties of $n_{z \ell}$ in (7d), we also have $\left\|z_{p}\right\|_{N_{z}} \leq \mathbf{z}_{\infty}$, with $\mathbf{z}_{\infty}=\frac{1}{\mu^{2} n_{z 1}} \mathbf{q}_{t}(0,0)$.

Now, let $\mathbf{e}$ be given by Proposition 4 , and select $\boldsymbol{\zeta}_{\infty}=2 \mathbf{z}_{\infty}+2 \bar{N}_{z} \mathbf{e}_{\infty}$. With such choice, we ensure that both initial conditions $z(0)=0$ and $z(0)=z_{p}$ belong the set $\Omega_{\text {init }}$ defined in (128) and are therefore included in the domain of attraction in view of item (ii) of Proposition 4. Indeed, following similar computations to those used in the proof of item 1) of Theorem 1, we can show that

$$
\left\{(x, e, z) \in \mathbb{R}^{n} \times \mathbb{R} \times \mathscr{L}_{N_{z}}^{2}:|x| \leq 2 \mathbf{x}_{\infty},|e| \leq 2 \mathbf{e}_{\infty},\|z\|_{N_{z}} \leq 2 \mathbf{z}_{\infty}\right\} \subseteq \Omega_{\text {init }} .
$$

Note that such initial conditions are of particular interest, since $z(0)=0$ corresponds to the origin of the control law (which is a quite natural choice when the steady-state solution for $z$ is unknown), while $z(0)=z_{p}$ corresponds to the origin of the closed-loop system (125). Now, by applying Proposition 3 and 4 , we are guaranteed that, for $\sigma>\sigma_{\infty}^{\star}$, the solution to system (125) to be complete forward in time. Furthermore, as $\zeta(t)$ is in $\mathscr{L}_{N_{z}}^{2}$ for all $t \geq 0$, and $M$ is in $\mathscr{L}_{N_{z}}^{2}$ by design, we conclude that also $z(t)$ is in $\mathscr{L}_{N_{z}}^{2}$ for all $t \geq 0$. Moreover, $x$ and $e$ converges asymptotically to zero. 
Finally, the last part of the proof consists in showing that the domain of attraction given by Theorem 1 with a finite-dimensional regulator, is not reduced in terms of $(x, e)$-coordinates with the infinitedimensional regulator. Moreover, for such a bound, we want also $\sigma_{\infty}^{\star}<\sigma^{\star}$. In turn, by entering the details of the proof of Theorem 1, this consists in showing that for Proposition 3 we can select the bounds $\mathbf{x}_{a}, \mathbf{e}_{a}$ and $\zeta_{a}$ given by Proposition 2, and then compare $\sigma_{a}^{\star}$ with the resulting $\sigma_{\infty}^{\star}$. To this end, it suffices first to note that the bounds in (131) are less conservatives than (97) for two main reasons: in the first pair of equations, the right term is less conservative since we have $\mathbf{x}_{b}$ (resp. $\mathbf{e}_{b}$ ) and not $\mathbf{x}_{b}-\mathbf{x}_{a}$ (resp. $\mathbf{e}_{b}-\mathbf{e}_{a}$ ); the second condition is less conservative in view of the definition of $\rho_{0}^{\infty}$ given in (129) compared to that of $\rho_{0}$ in (96). As a consequence, any selection of $\mathbf{x}_{a}, \mathbf{e}_{a}, \zeta_{a}$ given by Proposition 2 is a feasible choice for the statement of Proposition 4. This ensure that the domain of attraction in terms of $(x, e)$ coordinates for the infinite-dimensional regulator contains the domain of attraction obtained in the finite-dimensional case, and therefore that the bounds $\mathbf{x}, \mathbf{e}, \mathbf{z}$ of Theorem 2 can be taken from Theorem 1. Hence, to complete that statement of the theorem, it suffices to show that for a given set of values, $\sigma_{\infty}^{\star}<\sigma_{a}^{\star}$ given by Proposition 2 . Again, this can be easily proved by noting that the definition of $h$ in (132) is less conservative than the bound (98), and therefore, for a given $h$, we have $\sigma_{\infty}^{\star}<\sigma_{a}^{\star}$ since $\rho_{0}^{\infty}\left(\mathbf{x}_{b}\right)<\rho_{0}\left(\mathbf{x}_{a}, \mathbf{e}_{b}\right)$, see (99) and (133).

\section{Supplementary Material: a Technical Lemma}

Lemma 6 Let $\mu>0$ and let $n_{z \ell}$ be a sequences of positive real numbers satisfying (7a). There exist strictly positive real numbers $\kappa_{0}$ and $\kappa_{1}$ independent of $n_{o}$ such that the function $\mathcal{T}(x)$ defined as in (36), i.e.

$$
\mathcal{T}(x)=\frac{\sum_{\ell=0}^{n_{o}} \frac{\ell^{2}+x^{2}}{\left(\ell^{2}-x^{2}\right)^{2}} n_{z \ell}}{1+\mu^{2} x^{2}\left(\sum_{\ell=0}^{n_{o}} \frac{1}{\ell^{2}-x^{2}} n_{z \ell}\right)^{2}}
$$

satisfies

$$
\mathcal{T}(x) \leq \kappa_{0}+\kappa_{1} x^{2} \quad \forall x \in \mathbb{R}, n_{o} \in \mathbb{N}, \mu \geq 1 .
$$

Proof Seemingly the function $\mathcal{T}$ has singularities at $x=\ell$. But having :

$$
\mathcal{T}(x)=\frac{\sum_{\ell=0}^{n_{o}}\left(\ell^{2}+x^{2}\right) n_{z \ell}\left[\prod_{m=0, \neq \ell}^{n_{o}}\left(m^{2}-x^{2}\right)\right]^{2}}{\left[\prod_{m=0}^{n_{o}}\left(m^{2}-x^{2}\right)\right]^{2}+\mu^{2} x^{2}\left[\sum_{\ell=0}^{n_{o}} n_{z \ell} \prod_{m=0, \neq \ell}^{n_{o}}\left(m^{2}-x^{2}\right)\right]^{2}}
$$

we conclude that these singularities are fictitious and actually $\mathcal{T}$ is a $C^{\infty}$ function of $x$ on $\mathbb{R}$. Moreover we have

With (7d) this yields

$$
\mathcal{T}(\ell)=\frac{2}{\mu^{2} n_{z \ell}} \quad \forall \ell \in\left\{0, \ldots, n_{o}\right\} .
$$

$$
\mathcal{T}(\ell) \leq \frac{2 \ell^{2}}{\mu^{2} n_{z 1}} \quad \forall \ell \in\left\{0, \ldots, n_{o}\right\} .
$$

With this at hand, we continue in studying $\mathcal{T}$ for $x$ non integer.

To isolate the (fictitious) singularities at $x=m$ or $x=m+1$, with $m \in\left\{0, \ldots, n_{o}-1\right\}$, we consider the sums without these values by defining

$$
\begin{aligned}
S_{m}(x) & :=\underline{S}_{m}(x)-\bar{S}_{m}(x) \\
\underline{S}_{m}(x) & :=\sum_{\ell=0}^{m-1} \frac{1}{x^{2}-\ell^{2}} \frac{n_{z \ell}}{n_{z m}}, \quad \underline{S}_{0}(x)=0, \\
\bar{S}_{m}(x) & :=\sum_{\ell=m+2}^{n_{0}} \frac{1}{\ell^{2}-x^{2}} \frac{n_{z \ell}}{n_{z m}} \quad, \quad \bar{S}_{n_{o}-1}(x)=0,
\end{aligned}
$$


and

$$
Q_{m}(x):=\sum_{\ell=0, \neq m, \neq m+1}^{n_{0}} \frac{x^{2}+\ell^{2}}{\left(x^{2}-\ell^{2}\right)^{2}} n_{z \ell} .
$$

To simplify these expressions, we introduce the notation

$$
z(\ell):=\frac{n_{z(\ell+1)}}{n_{z \ell}} .
$$

With (7b) and (7d), we have

$$
\frac{1}{4} \leq \frac{\ell^{2}}{(\ell+1)^{2}} \leq r(\ell)<1 \quad \forall 0<\ell .
$$

Then (36) is simplified into

$$
\mathcal{T}(x)=F_{n_{o}}(x)+G_{n_{o}}(x)
$$

where

$$
\begin{aligned}
F_{n_{o}}(x) & :=\frac{n_{z m}\left[\frac{x^{2}+m^{2}}{\left(x^{2}-m^{2}\right)^{2}}+\frac{x^{2}+(m+1)^{2}}{\left((m+1)^{2}-x^{2}\right)^{2}} z(m)\right]}{1+\mu^{2} x^{2} n_{z m}^{2}\left(S_{m}(x)+\frac{1}{x^{2}-m^{2}}-\frac{1}{(m+1)^{2}-x^{2}} z(m)\right)^{2}}, \\
G_{n_{o}}(x) & :=\frac{Q_{m}(x)}{1+\mu^{2} x^{2} n_{z m}^{2}\left(S_{m}(x)+\frac{1}{x^{2}-m^{2}}-\frac{1}{(m+1)^{2}-x^{2}} z(m)\right)^{2}} .
\end{aligned}
$$

Let now study the behavior of the functions $F_{n_{o}}, G_{n_{o}}$. We divide the analysis into three cases, where, in any case, $x$ is not an integer

a) $0<x<n_{o}$

b) $n_{o}<x<n_{o}+1$,

c) $n_{o}+1 \leq x$.

Case a) $0<x<n_{o}$. Then there exists $m \in\left\{0, \ldots, n_{o}-1\right\}$ such that $x \in(m, m+1)$. First, we look for a bound for $\bar{S}_{m}(m)$. With (7c), this gives

$$
\bar{S}_{m}(m) \leq m \sum_{\ell=m+2}^{n_{o}} \frac{1}{\ell^{2}-m^{2}} \frac{1}{\ell}
$$

We have

$$
\frac{1}{\ell^{2}-m^{2}} \frac{1}{\ell}=\frac{1}{m^{2}}\left[\frac{1}{2(\ell-m)}+\frac{1}{2(\ell+m)}-\frac{1}{\ell}\right]
$$

Since the function $s \mapsto \frac{1}{2(s-m)}+\frac{1}{2(s+m)}-\frac{1}{s}$ is decreasing on $\left[\frac{m}{\sqrt{3}},+\infty[\right.$, we get

$$
\begin{aligned}
\bar{S}_{m}(m) & \leq \frac{1}{m} \int_{m+1}^{\infty}\left[\frac{1}{2(s-m)}+\frac{1}{2(s+m)}-\frac{1}{s}\right] d s \\
& \leq\left.\frac{1}{m} \log \left(\frac{\sqrt{s^{2}-m^{2}}}{s}\right)\right|_{m+1} ^{\infty} \\
& \leq \frac{1}{m} \log \left(\frac{m+1}{\sqrt{2 m+1}}\right) \leq \frac{1}{m} \log (\sqrt{m+1}) \leq \frac{1}{2} .
\end{aligned}
$$

So the sequence $\bar{S}_{m}(m)$ is bounded independently of $n_{o}$.

Then, we look for a bound for $\underline{S}_{m}(m)$. For $m \geq 2$, we have the decomposition

$$
\underline{S}_{m}(m)=\sum_{\ell=0}^{m-1} \frac{1}{m^{2}-\ell^{2}} \frac{n_{z \ell}}{n_{z m}}
$$


With $(7 \mathrm{~d})$, this gives

$$
\underline{S}_{m}(m) \leq \frac{1}{m^{2}} \frac{n_{z 0}}{n_{z m}}+\frac{1}{m^{2}-1} \frac{n_{z 1}}{n_{z m}}+\sum_{\ell=2}^{m-2} \frac{1}{m^{2}-\ell^{2}} \frac{m^{2}}{\ell^{2}}+\frac{1}{m^{2}-(m-1)^{2}} \frac{n_{z(m-1)}}{n_{z m}}
$$

Since the function $s \mapsto \frac{1}{m^{2}-s^{2}} \frac{m^{2}}{s^{2}}$ is decreasing on $\left.] 0, \frac{m}{\sqrt{2}}\right]$ and increasing on $\left[\frac{m}{\sqrt{2}}, m[\right.$, we have

$$
\sum_{\ell=2}^{m-2} \frac{1}{m^{2}-\ell^{2}} \frac{m^{2}}{\ell^{2}} \leq \int_{1}^{m-1} \frac{1}{m^{2}-s^{2}} \frac{m^{2}}{s^{2}} d s
$$

Then, with

$$
\frac{1}{m^{2}-s^{2}} \frac{m^{2}}{s^{2}}=\frac{1}{s^{2}}+\frac{1}{2 m}\left(\frac{1}{m-s}+\frac{1}{m+s}\right)
$$

we obtain

$$
\begin{aligned}
\int_{1}^{m-1} \frac{1}{m^{2}-s^{2}} \frac{m^{2}}{s^{2}} d s & =\left.\left[-\frac{1}{s}+\frac{1}{2 m} \log \left(\frac{m+s}{m-s}\right)\right]\right|_{1} ^{m-1} \\
& =1-\frac{1}{m-1}+\frac{1}{2 m} \log \left(\frac{(2 m-1)(m-1)}{m+1}\right)
\end{aligned}
$$

This yields

$$
\begin{aligned}
\underline{S}_{m}(m) & \leq \frac{1}{m^{2}} \frac{n_{z 0}}{n_{z m}}+\frac{1}{m^{2}-1} \frac{n_{z 1}}{n_{z m}}+\left[\frac{m-2}{m-1}+\frac{1}{2 m} \log \left(\frac{(2 m-1)(m-1)}{m+1}\right)\right]+\frac{1}{m^{2}-(m-1)^{2}} \frac{n_{z(m-1)}}{n_{z m}}, \\
& \leq \frac{1}{m^{2}} \frac{n_{z 0}}{n_{z m}}+\frac{1}{m^{2}-1} \frac{n_{z 1}}{n_{z m}}+2+\frac{1}{m^{2}-(m-1)^{2}} \frac{n_{z(m-1)}}{n_{z m}}
\end{aligned}
$$

But with (7d), (7b) and $m \geq 2$, we have

$$
\frac{1}{m^{2}} \frac{n_{z 0}}{n_{z m}} \leq \frac{n_{z 0}}{n_{z 1}} \quad, \quad \frac{1}{m^{2}-1} \frac{n_{z 1}}{n_{z m}} \leq \frac{n_{z 0}}{3 n_{z 2}} \quad, \quad \frac{1}{m^{2}-(m-1)^{2}} \frac{n_{z(m-1)}}{n_{z m}} \leq \frac{m^{2}}{(m-1)^{2}(2 m-1)} \leq \frac{4}{3}
$$

Hence, $\underline{S}_{m}(m)$ is bounded independently of $n_{o}$ as

$$
\underline{S}_{m}(m) \leq \frac{n_{z 0}}{n_{z 1}}+\frac{n_{z 0}}{3 n_{z 2}}+\frac{10}{3}
$$

In conclusion we have obtained the real number

$$
\mathbf{S}=\frac{n_{z 0}}{n_{z 1}}+\frac{n_{z 0}}{3 n_{z 2}}+\frac{10}{3}
$$

independent of $n_{o}$, such that

$$
\left|S_{m}(x)\right| \leq\left|\sum_{\ell=0}^{m-1} \frac{1}{m^{2}-\ell^{2}} \frac{n_{z \ell}}{n_{z m}}-\sum_{\ell=m+2}^{n_{o}} \frac{1}{\ell^{2}-m^{2}} \frac{n_{z \ell}}{n_{z m}}\right| \leq \mathbf{S} \quad \forall m \in\left\{0, \ldots, n_{o}-1\right\}, \quad \forall n_{o}
$$

Finally we look for a bound for $Q_{m}(x)$. We decompose, with no care about the boundary effects for $m<2$ or $m>n_{o}-3$,

$$
\begin{aligned}
Q_{m}(x)=\sum_{\ell=0}^{m-2} \frac{x^{2}+\ell^{2}}{\left(x^{2}-\ell^{2}\right)^{2}} n_{z \ell} & \\
& +\frac{x^{2}+(m-1)^{2}}{\left(x^{2}-(m-1)^{2}\right)^{2}} n_{z(m-1)}+\frac{x^{2}+(m+2)^{2}}{\left(x^{2}-(m+2)^{2}\right)^{2}} n_{z(m+2)}+\sum_{\ell=m+3}^{n_{0}} \frac{x^{2}+\ell^{2}}{\left(x^{2}-\ell^{2}\right)^{2}} n_{z \ell}
\end{aligned}
$$


With (7b), since the function $s \mapsto \frac{s^{2}+1}{\left(s^{2}-1\right)^{2}}$ is increasing for $s<1$ and decreasing for $1<s$, and $x$ is in ] $m, m+1$ [, we get, for $m \geq 1$,

$$
\frac{Q_{m}(x)}{n_{z 0}} \leq \int_{0}^{m-1} \frac{m^{2}+\ell^{2}}{\left(m^{2}-\ell^{2}\right)^{2}} d \ell+\frac{2 m^{2}-2 m+1}{(2 m-1)^{2}}+\frac{2 m^{2}+5 m+5}{(2 m+3)^{2}}+\int_{m+2}^{\infty} \frac{(m+1)^{2}+\ell^{2}}{\left((m+1)^{2}-\ell^{2}\right)^{2}} d \ell
$$

Using the identity

$$
\frac{s^{2}+a^{2}}{\left(s^{2}-a^{2}\right)^{2}}=-\frac{d}{d s}\left\{\frac{s}{s^{2}-a^{2}}\right\}
$$

we obtain

$$
\begin{aligned}
\int_{0}^{m-1} \frac{m^{2}+\ell^{2}}{\left(m^{2}-\ell^{2}\right)^{2}} d \ell & =\frac{m-1}{m^{2}-(m-1)^{2}}=\frac{m-1}{2 m-1} \\
\int_{m+2}^{\infty} \frac{(m+1)^{2}+\ell^{2}}{\left((m+1)^{2}-\ell^{2}\right)^{2}} d \ell & =\frac{m+2}{\left((m+2)^{2}-(m+1)^{2}\right)}=\frac{m+2}{2 m+3}
\end{aligned}
$$

and therefore

$$
\frac{Q_{m}(x)}{n_{z 0}} \leq \frac{m-1}{2 m-1}+\frac{2 m^{2}-2 m+1}{(2 m-1)^{2}}+\frac{2 m^{2}+5 m+5}{(2 m+3)^{2}}+\frac{m+2}{2 m+3} \leq \frac{7}{2}
$$

We have also

$$
Q_{0}(x)=\sum_{\ell=2}^{n_{0}} \frac{x^{2}+\ell^{2}}{\left(x^{2}-\ell^{2}\right)^{2}} n_{z \ell} \leq n_{z 2} \sum_{\ell=2}^{n_{0}} \frac{1+\ell^{2}}{\left(1-\ell^{2}\right)^{2}} \leq n_{z 2}\left[\frac{5}{9}+\int_{2}^{\infty} \frac{1+\ell^{2}}{\left(1-\ell^{2}\right)^{2}} d \ell\right] \leq n_{z 2} \frac{11}{9},
$$

implying $Q_{0}(x) \leq Q_{m}(x)$ with (7b). This gives readily the following bound for $\left|G_{n_{o}}(x)\right|$

$$
\left|G_{n_{o}}(x)\right| \leq Q_{m}(x) \leq \frac{7}{2} n_{z 0}
$$

As desired this bound is independent of $n_{o}, m$

With all this, to obtain a bound for $\left|F_{n_{o}}(x)\right|$, we start by simplifying the expression of the denominator in (143). For this, we define the following change of variables

$$
z=\Psi(x):=-\frac{1}{X-m^{2}}+\frac{r(m)}{(m+1)^{2}-X},
$$

where $X=x^{2}$. Since $x \in(m, m+1)$, we decompose the interval $\mathfrak{X}:=\left(m^{2},(m+1)^{2}\right)$ as $\mathfrak{X}=\mathfrak{X}_{-} \cup \mathfrak{X}_{0} \cup \mathfrak{X}_{+}$ where

$$
\begin{aligned}
\mathfrak{X}_{-} & =\left(m^{2}, X_{a}\right] & X_{a} & =(1-\lambda) m^{2}+\lambda(m+1)^{2}=m^{2}+\lambda(2 m+1) \\
\mathfrak{X}_{0} & =\left[X_{a}, X_{b}\right] & & X_{b}=\lambda m^{2}+(1-\lambda)(m+1)^{2}=m^{2}+(1-\lambda)(2 m+1) \\
\mathfrak{X}_{+} & =\left[X_{b},(m+1)^{2}\right) & &
\end{aligned}
$$

with $\lambda \in\left(0, \frac{1}{2}\right]$ to be selected with in mind the fact that when $\lambda$ is close to $0, X_{a}$ is close to $m^{2}$ and $X_{b}$ close to $(m+1)^{2}$. These sets are mapped, via $\Psi$, into the sets

with the notation

$$
\begin{array}{rlrl}
\mathfrak{Z}_{-} & =\left(-\infty, \mathfrak{z}_{a}\right] & \mathfrak{z}_{a} & =-\frac{(1-\lambda) \mathfrak{m}_{p}-\lambda z(m) \mathfrak{m}_{m}}{\lambda(1-\lambda) \mathfrak{m}_{p} \mathfrak{m}_{m}} \\
\mathfrak{Z}_{0} & =\left[\mathfrak{z}_{a}, \mathfrak{z}_{b}\right] & & \\
\mathfrak{Z}_{+} & =\left[\mathfrak{z}_{b},+\infty\right) & \mathfrak{z}_{b}=\left(\frac{\tau(m)}{1+\tau(m)}-\lambda\right) \frac{1+\tau(m)}{\lambda(1-\lambda)} \frac{1}{\mathfrak{m}_{m}}
\end{array}
$$

$$
\mathfrak{m}_{p}=\left[(m+1)^{2}+m^{2}\right] \quad \mathfrak{m}_{m}=\left[(m+1)^{2}-m^{2}\right]=2 m+1
$$

Note that function $\Psi$ is injective and its inverse transformation is continuous and given by

$$
\begin{aligned}
& X(z)=\frac{\mathfrak{m}_{p} z-(1+z(m))+\sqrt{\left(z \mathfrak{m}_{m}+(1-z(m))\right)^{2}+4 r(m)}}{2 z} \quad \text { if } z \neq 0 \\
& X(0)=\frac{(m+1)^{2}+m^{2} z(m)}{1+z(m)}
\end{aligned}
$$

About the choice of $\lambda$, we gather here all the constraints we shall impose later on. 
1. We want $X(0)$ be in $\mathfrak{X}_{0}$, or equivalently $\mathfrak{z}_{a}<0<\mathfrak{z}_{b}$. This motivates the following inequalities

$$
X_{a}=m^{2}+\lambda(2 m+1)<m^{2}+(2 m+1) \frac{1}{1+\tau(m)}<m^{2}+(1-\lambda)(2 m+1)=X_{b}
$$

This is always verified for any $m \geq 1$ if we select

$$
\lambda<\frac{1}{1+\tau(m)}<1-\lambda
$$

With (141), that is

$$
\lambda<\frac{1}{5}
$$

2. With (146), the triangle inequality gives

$$
\left|S_{m}(x)+z\right| \geq|z|-\mathbf{S}
$$

We want to have

$$
\left|S_{m}(x)+z\right| \geq|z|-\mathbf{S} \geq \frac{1}{\sqrt{2}}|z| \quad \forall z \in \mathfrak{Z}_{-} \cup \mathfrak{Z}_{+}
$$

We want also

$$
\frac{(1+z(m))^{2}}{2 \tau(m) \mathfrak{m}_{m}} \leq|z| \quad \forall z \in \mathfrak{Z}_{-} \cup \mathfrak{Z}_{+}
$$

This holds if

$$
\max \left\{(2+\sqrt{2}) \mathbf{S}, \frac{25}{8}\right\} \leq \min \{-\mathfrak{z} a, \mathfrak{z} b\}
$$

Let

$$
\mathfrak{a}=\max \left\{(2+\sqrt{2}) \mathbf{S}, \frac{25}{8}\right\} .
$$

With (145), this number does not depend on $n_{o}$. We have $-\mathfrak{z} a \geq \mathfrak{a}$ if

$$
\frac{(1-\lambda) \mathfrak{m}_{p}-\lambda z(m) \mathfrak{m}_{m}}{\lambda(1-\lambda) \mathfrak{m}_{p} \mathfrak{m}_{m}} \geq \mathfrak{a}
$$

which gives

$$
\lambda^{2}-\left(1+\frac{\mathfrak{m}_{p}+\tau(m) \mathfrak{m}_{m}}{\mathfrak{m}_{p} \mathfrak{m}_{m} \mathfrak{a}}\right) \lambda+\frac{1}{\mathfrak{m}_{m} \mathfrak{a}} \geq 0
$$

and by recalling that $\lambda \in\left(0, \frac{1}{2}\right]$, we obtain that the inequality is verified for $\lambda \in\left(0, \lambda_{a}\right]$ when

$$
\lambda_{a}=\frac{1}{2}\left(1+\frac{\mathfrak{m}_{p}+z(m) \mathfrak{m}_{m}}{\mathfrak{m}_{p} \mathfrak{m}_{m} \mathfrak{a}}\right)-\frac{1}{2} \sqrt{\left(1+\frac{\mathfrak{m}_{p}+r(m) \mathfrak{m}_{m}}{\mathfrak{m}_{p} \mathfrak{m}_{m} \mathfrak{a}}\right)^{2}-\frac{4}{\mathfrak{m}_{m} \mathfrak{a}}}
$$

Similarly, we have $\mathfrak{z} b \geq \mathfrak{a}$ if

$$
\left(\frac{\tau(m)}{1+\tau(m)}-\lambda\right) \frac{1+\tau(m)}{\lambda(1-\lambda)} \frac{1}{\mathfrak{m}_{m}} \geq \mathfrak{a}
$$

which gives

$$
\lambda^{2}-\left(1+\frac{1+z(m)}{\mathfrak{a m}_{m}}\right) \lambda+\frac{z(m)}{\mathfrak{a m}_{m}} \geq 0
$$

which is satisfied for all $\lambda \in\left(0, \lambda_{b}\right]$ when

$$
\lambda_{b}=\frac{1}{2}\left(1+\frac{1+r(m)}{\mathfrak{a m}_{m}}\right)-\frac{1}{2} \sqrt{\left(1+\frac{1+\tau(m)}{\mathfrak{m}_{m} \mathfrak{a}}\right)^{2}-\frac{\tau(m)}{\mathfrak{m}_{m} \mathfrak{a}}}
$$


Now, we look for a lower bound for $\lambda_{a}, \lambda_{b}$. To this end, note that they satisfy

$$
\begin{aligned}
\lambda_{i} & =\frac{1}{2}\left[T_{1 i}-\sqrt{T_{1 i}^{2}-T_{2 i}}\right]=\frac{1}{2} \frac{T_{1 i}^{2}-\left(\sqrt{T_{1 i}^{2}-T_{2 i}}\right)^{2}}{T_{1 i}+\sqrt{T_{1 i}^{2}-T_{2 i}}} \\
& =\frac{1}{2} \frac{T_{2 i}}{T_{1 i}+\sqrt{T_{1 i}^{2}-T_{2 i}}} \\
& \geq \frac{1}{4} \frac{T_{2 i}}{T_{1 i}}
\end{aligned}
$$

where

By further using

$$
\begin{aligned}
T_{1 a} & =1+\frac{\mathfrak{m}_{p}+z(m) \mathfrak{m}_{m}}{\mathfrak{m}_{p} \mathfrak{m}_{m} \mathfrak{a}}, & & T_{2 a}=\frac{4}{\mathfrak{m}_{m} \mathfrak{a}} \\
T_{1 b} & =1+\frac{1+\tau(m)}{\mathfrak{m}_{m} \mathfrak{a}}, & T_{2 b} & =\frac{z(m)}{\mathfrak{m}_{m} \mathfrak{a}}
\end{aligned}
$$

$$
T_{1 a}=1+\frac{1}{\mathfrak{m}_{m} \mathfrak{a}}+\frac{z(m)}{\mathfrak{m}_{p} \mathfrak{a}} \leq 1+\frac{1}{\mathfrak{a}}+\frac{1}{\mathfrak{a}}=\frac{2+\mathfrak{a}}{\mathfrak{a}}
$$

and similarly,

$$
T_{1 b} \leq \frac{2+\mathfrak{a}}{\mathfrak{a}}
$$

we finally obtain

$$
\begin{aligned}
& \lambda_{a} \geq \frac{1}{2+\mathfrak{a}} \frac{1}{\mathfrak{m}_{m}}, \\
& \lambda_{b} \geq \frac{1}{4} \frac{z(m)}{2+\mathfrak{a}} \frac{1}{\mathfrak{m}_{m}} \geq \frac{1}{16} \frac{1}{2+\mathfrak{a}} \frac{1}{2 m+1} .
\end{aligned}
$$

Since $\mathfrak{a}$ does not depend on $m$ nor $n_{o}$, by selecting $\lambda$, depending on $m \geq 1$ and satisfying

$$
\frac{1}{5} \geq \lambda \geq \frac{\varpi}{m}, \quad \varpi:=\frac{1}{48(\mathfrak{a}+2)}
$$

we have always satisfied $\lambda \in\left(0, \min \left\{\lambda_{a}, \lambda_{b}, \frac{1}{5}\right\}\right]$. Note that, according to (153) and (145), $\varpi$ does not depend on $m$ or $n_{o}$.

3. We want to have

$$
\sqrt{\left(z \mathfrak{m}_{m}+(1-r(m))\right)^{2}+4 r(m)} \geq(1+r(m)) \quad \forall z \in \mathfrak{Z}_{-} \cup \mathfrak{Z}_{+}
$$

This holds if $z$ is not in $]-2 \frac{1-z(m)}{\mathfrak{m}_{m}}, 0[$ and therefore if we have

$$
-\frac{(1-\lambda) \mathfrak{m}_{p}-\lambda z(m) \mathfrak{m}_{m}}{\lambda(1-\lambda) \mathfrak{m}_{p} \mathfrak{m}_{m}}=\mathfrak{z}_{a} \leq-2 \frac{1-\tau(m)}{\mathfrak{m}_{m}}
$$

This inequality reduces to

$$
2[1-z(m)] \mathfrak{m}_{p} \lambda^{2}-\left(\mathfrak{m}_{p}+r(m) \mathfrak{m}_{m}+2[1-z(m)] \mathfrak{m}_{p}\right) \lambda+\mathfrak{m}_{p} \geq 0
$$

It is satisfied for all $\lambda \in\left(0, \min \left\{\frac{1}{2}, \lambda_{c}\right\}\right]$ with

$$
\lambda_{c}=\frac{1}{2}\left(1+\frac{\mathfrak{m}_{p}+z(m) \mathfrak{m}_{m}}{2[1-\tau(m)] \mathfrak{m}_{p}}\right)-\frac{1}{2} \sqrt{\left(1+\frac{\mathfrak{m}_{p}+\tau(m) \mathfrak{m}_{m}}{2[1-z(m)] \mathfrak{m}_{p}}\right)^{2}-\frac{2}{1-\tau(m)}} .
$$

By following similar computations to those made for $\lambda_{a}, \lambda_{b}$, previous expression gives also

$$
\lambda_{c} \geq \frac{1}{2[1-\tau(m)]} \frac{1}{1+\frac{\mathfrak{m}_{p}+\tau(m) \mathfrak{m}_{m}}{2[1-\tau(m)] \mathfrak{m}_{p}}} \geq \frac{1}{3-\tau(m)\left[2-\frac{\mathfrak{m}_{m}}{\mathfrak{m}_{p}}\right]} \geq \frac{1}{3} .
$$


Collecting all these constraints, we conclude that $\lambda$ can be selected in the interval

$$
\frac{\varpi}{m}=\frac{1}{48(\mathfrak{a}+2)} \frac{1}{m} \leq \lambda \leq \frac{1}{5}
$$

After these preliminaries, we are ready to derive bounds for $\left|F_{n_{o}}(x)\right|$. For the case where $x$ is in $\mathfrak{X}_{0}$ or equivalently $z$ in $\mathfrak{Z}_{0}$, we have

$$
\left(x^{2}-m^{2}\right)^{2} \geq\left(X_{a}-m^{2}\right)^{2} \quad, \quad\left((m+1)^{2}-x^{2}\right)^{2} \geq\left((m+1)^{2}-X_{b}\right)^{2} .
$$

This gives

$$
\begin{aligned}
\left|F_{n_{o}}(x)\right| & \leq n_{z m}\left[\frac{x^{2}+m^{2}}{\left(x^{2}-m^{2}\right)^{2}}+\frac{x^{2}+(m+1)^{2}}{\left((m+1)^{2}-x^{2}\right)^{2}} z(m)\right] \\
& \leq 2 n_{z m}(m+1)^{2}\left[\frac{1}{\left(X_{a}-m^{2}\right)^{2}}+\frac{1}{\left((m+1)^{2}-X_{b}\right)^{2}} z(m)\right] \\
& \leq 2 n_{z m}(m+1)^{2}\left[\frac{1}{\lambda^{2}(2 m+1)^{2}}+\frac{1}{(1-\lambda)^{2}(2 m+1)^{2}}\right] \\
& \leq 2 n_{z 0}\left(\frac{m+1}{2 m+1}\right)^{2}\left[\frac{1}{\lambda^{2}}+\frac{1}{(1-\lambda)^{2}}\right]
\end{aligned}
$$

So, with (156), we get

$$
\left|F_{n_{o}}(x)\right| \leq \frac{4 n_{z 0}}{\varpi^{2}} m^{2} \quad \forall x \in \mathfrak{X}_{0} .
$$

For the case where $x$ is in $\mathfrak{X}_{-} \cup \mathfrak{X}_{+}$, or equivalently $z$ is in $\mathfrak{Z}_{-} \cup \mathfrak{Z}_{+}$, we have, with (148),

$$
x^{2}-m^{2}=\frac{z \mathfrak{m}_{m}-c(z)}{2 z}, \quad(m+1)^{2}-x^{2}=\frac{z \mathfrak{m}_{m}+c(z)}{2 z}
$$

with the notation

$$
c(z)=1+z(m)-\sqrt{\left(z \mathfrak{m}_{m}+(1-z(m))\right)^{2}+4 z(m)} .
$$

where $c(z)$ is non positive according to (155). So, by using $z(m) \leq 1$ and $m<x<m+1$, and $\mathfrak{m}_{m}=2 m+1$, we obtain

$$
\left|F_{n_{o}}(x)\right| \leq \frac{8 n_{z m}(m+1)^{2}\left[\frac{z^{2}}{\left(z \mathfrak{m}_{m}-c(z)\right)^{2}}+\frac{z^{2}}{\left(z \mathfrak{m}_{m}+c(z)\right)^{2}}\right]}{1+\mu^{2} m^{2} n_{z m}^{2}\left(S_{m}(x)+z\right)^{2}}
$$

But (151) gives

$$
\begin{aligned}
\frac{n_{z m}(m+1)^{2} z^{2}}{1+\mu^{2} m^{2} n_{z m}^{2}\left(S_{m}(x)+z\right)^{2}} & \leq n_{z 0} \quad \text { if } m=0 \\
& \leq \frac{2 n_{z m}(m+1)^{2} z^{2}}{2+\mu^{2} m^{2} n_{z m}^{2} z^{2}} \quad \forall z \in \mathfrak{Z}_{-} \cup \mathfrak{Z}_{+} \quad \text { if } m \geq 1 \\
& \leq \frac{1}{n_{z m}} \frac{(m+1)^{2}}{m^{2}} \frac{2}{\mu^{2}} \quad \forall z \in \mathfrak{Z}_{-} \cup \mathfrak{Z}_{+} \quad \text { if } m \geq 1
\end{aligned}
$$

This yields

$$
\begin{aligned}
& \left|F_{n_{o}}(x)\right| \leq \frac{1}{n_{z m}} \frac{(m+1)^{2}}{m^{2}} \frac{32}{\mu^{2}} \frac{\left(z \mathfrak{m}_{m}\right)^{2}+c(z)^{2}}{\left(\left(z \mathfrak{m}_{m}\right)^{2}-c(z)^{2}\right)^{2}} \quad \forall z \in \mathfrak{Z}_{-} \cup \mathfrak{Z}_{+} \\
& \left|F_{n_{o}}(x)\right| \leq \frac{1}{n_{z m}} \frac{32}{\mu^{2}} \frac{1}{z^{2} \mathfrak{m}_{m}^{2}} \frac{1+\left[\frac{c(z)}{z \mathfrak{m}_{m}}\right]^{2}}{\left(1-\left[\frac{c(z)}{z \mathfrak{m}_{m}}\right]^{2}\right)^{2}} \quad \forall z \in \mathfrak{Z}_{-} \cup \mathfrak{Z}_{+}
\end{aligned}
$$


Since the function $\chi^{2} \mapsto \frac{1+\chi^{2}}{\left(1-\chi^{2}\right)^{2}}$ is non decreasing on [0,1[ we go on by looking for an upperbund for $\left|\frac{c(z)}{z \mathfrak{m}_{m}}\right|$. With (155), we have

$$
\left|\frac{c(z)}{z \mathfrak{m}_{m}}\right|=\frac{\sqrt{\left(z \mathfrak{m}_{m}+(1-z(m))\right)^{2}+4 r(m)}-(1+z(m))}{|z| \mathfrak{m}_{m}}
$$

Then, the inequality

$$
\sqrt{1+a} \leq 1+\frac{a}{2} \quad \forall a \geq-1
$$

allows us to write

$$
\left|\frac{c(z)}{z \mathfrak{m}_{m}}\right| \leq 1+\frac{1-z(m)}{z \mathfrak{m}_{m}}+\frac{(1+\tau(m))^{2}}{2 z^{2} \mathfrak{m}_{m}^{2}}-\frac{1+z(m)}{|z| \mathfrak{m}_{m}} .
$$

But on one hand, we have

$$
\begin{array}{rlrl}
\frac{1-z(m)}{z \mathfrak{m}_{m}}-\frac{1+z(m)}{|z| \mathfrak{m}_{m}} & =-\frac{2}{|z| \mathfrak{m}_{m}} \leq-\frac{2 \tau(m)}{|z| \mathfrak{m}_{m}} & \text { if } z<0, \\
& =-\frac{2 z(m)}{|z| \mathfrak{m}_{m}} \quad \text { if } 0<z .
\end{array}
$$

On another hand, we have :

$$
\frac{(1+z(m))^{2}}{2 z^{2} \mathfrak{m}_{m}{ }^{2}} \leq \frac{z(m)}{|z| \mathfrak{m}_{m}} \quad \text { if } \quad \frac{(1+z(m))^{2}}{2 z(m) \mathfrak{m}_{m}} \leq|z| .
$$

This yields

$$
\left|\frac{c(z)}{z \mathfrak{m}_{m}}\right| \leq 1-\frac{z(m)}{|z| \mathfrak{m}_{m}} \quad \text { if } \quad \frac{(1+z(m))^{2}}{2 z(m) \mathfrak{m}_{m}} \leq|z| .
$$

So, with (152), we have obtained

$$
\left|F_{n_{o}}(x)\right| \leq \frac{1}{n_{z m}} \frac{32}{\mu^{2}} \frac{1}{z^{2} \mathfrak{m}_{m}^{2}} \frac{1+\left[1-\frac{z(m)}{z \mid \mathfrak{m}_{m}}\right]^{2}}{\left(1-\left[1-\frac{z(m)}{|z| \mathfrak{m}_{m}}\right]^{2}\right)^{2}}=\frac{1}{n_{z m}} \frac{32}{\mu^{2}} \frac{1}{\tau(m)^{2}} \frac{1+\left[1-\frac{z(m)}{z \mid \mathfrak{m}_{m}}\right]^{2}}{\left[2-\frac{z(m)}{|z| \mathfrak{m}_{m}}\right]^{2}} \quad \forall z \in \mathfrak{Z}_{-} \cup \mathfrak{Z}_{+} .
$$

We have

and, because of (141),

$$
\frac{1+[1-\chi]^{2}}{[2-\chi]^{2}} \leq 1 \quad \forall \chi \leq 1
$$

$$
\frac{z(m)}{|z| \mathfrak{m}_{m}} \leq \frac{(1+z(m))^{2}}{2 z(m)|z| \mathfrak{m}_{m}} \leq 1 \quad \forall z: \frac{(1+z(m))^{2}}{2 z(m) \mathfrak{m}_{m}} \leq|z| .
$$

We conclude that (152) gives finally

$$
\left|F_{n_{o}}(x)\right| \leq \frac{512}{\mu^{2}} \frac{1}{n_{z m}} \quad \forall z \in \mathfrak{Z}_{-} \cup \mathfrak{Z}_{+} .
$$

Hence, by putting together (157) and (158) we obtain

$$
\left|F_{n_{o}}(x)\right| \leq \max \left\{\frac{4 n_{z 0}}{\varpi^{2}} m^{2}, \frac{512}{\mu^{2}} \frac{1}{n_{z m}}\right\} \quad \forall x \in(m, m+1) .
$$

Finally, by using (7d), (142), (147), and (159), we obtain, for each $x$ in $(m, m+1)$ with $m$ in $\left\{0, \ldots, n_{o}-1\right\}$,

$$
\begin{aligned}
\mathcal{T}(x) & \leq\left(\frac{7}{2} n_{z 0}+\frac{4 n_{z 0}}{\varpi^{2}} m^{2}+\frac{512}{\mu^{2}} \frac{1}{n_{z m}}\right) \\
& \leq\left(\frac{7}{2} n_{z 0}+\left[\frac{4 n_{z 0}}{\varpi^{2}}+\frac{512}{\mu^{2}} \frac{1}{n_{z 1}}\right] m^{2}\right), \\
& \leq\left(\frac{7}{2} n_{z 0}+\left[\frac{4 n_{z 0}}{\varpi^{2}}+\frac{512}{\mu^{2}} \frac{1}{n_{z 1}}\right] x^{2}\right) .
\end{aligned}
$$


Case b) $n_{o}<x<n_{o}+1$. This case is a variant of the preceding one, with a (fictitious) singularity on left side only. In particular, the expression (36) simplifies as

$$
\mathcal{T}(x)=G_{n_{o}}(x)+F_{n_{o}}(x)
$$

where

$$
G_{n_{o}}(x):=\frac{\sum_{\ell=0}^{n_{o}-1} \frac{\ell^{2}+x^{2}}{\left(x^{2}-\ell^{2}\right)^{2}} n_{z \ell}}{1+\mu^{2} x^{2}\left(\sum_{\ell=0}^{n_{o}} \frac{1}{x^{2}-\ell^{2}} n_{z \ell}\right)^{2}}, \quad F_{n_{o}}(x):=\frac{\frac{n_{o}^{2}+x^{2}}{\left(x^{2}-n_{o}^{2}\right)^{2}} n_{z n_{0}}}{1+\mu^{2} x^{2}\left(\sum_{\ell=0}^{n_{o}} \frac{1}{x^{2}-\ell^{2}} n_{z \ell}\right)^{2}} .
$$

Concerning $G_{n_{o}}$, by recalling that $n_{z 0}>n_{z \ell}$, for any $\ell \geq 0$, and that the function $\chi \mapsto \frac{\chi+1}{(\chi-1)^{2}}$ is increasing on $[0,1[$ and decreasing on $] 1,+\infty[$, we compute

$$
\begin{aligned}
\left|G_{n_{o}}(x)\right| & \leq \sum_{\ell=0}^{n_{o}-1} \frac{\ell^{2}+x^{2}}{\left(\ell^{2}-x^{2}\right)^{2}} n_{z \ell} \\
& \leq\left[\sum_{\ell=0}^{n_{o}-2} \frac{\ell^{2}+n_{o}^{2}}{\left(\ell^{2}-n_{o}^{2}\right)^{2}}+\frac{\left(n_{o}-1\right)^{2}+n_{o}^{2}}{\left(\left(n_{o}-1\right)^{2}-n_{o}^{2}\right)^{2}}\right] n_{z 0} \\
& \leq\left[\int_{0}^{n_{o}-1} \frac{\ell^{2}+n_{o}^{2}}{\left(\ell^{2}-n_{o}^{2}\right)^{2}} d \ell+\frac{\left(n_{o}-1\right)^{2}+n_{o}^{2}}{\left(2 n_{o}-1\right)^{2}}\right] n_{z 0} \\
& \leq\left[\frac{n_{o}-1}{2 n_{o}-1}+\frac{\left(n_{o}-1\right)^{2}+n_{o}^{2}}{\left(2 n_{o}-1\right)^{2}}\right] n_{z 0} \leq n_{z 0} .
\end{aligned}
$$

Then, concerning $F_{n_{o}}$, we note that, since $x>n_{o}$, all the terms in the sum of the denominator are positive. As a consequence, we can write

$$
\left.F_{n_{o}}(x) \leq \frac{\frac{n_{o}^{2}+x^{2}}{\left(x^{2}-n_{o}^{2}\right)^{2}} n_{z n_{0}}}{1+\mu^{2} \frac{x^{2}}{\left(x^{2}-n_{o}^{2}\right)^{2}} n_{z n_{0}}^{2}} \leq \frac{\frac{2 x^{2}}{\left(x^{2}-n_{o}^{2}\right)^{2}} n_{z n_{0}}}{\mu^{2} \frac{x^{2}}{\left(x^{2}-n_{o}^{2}\right)^{2}} n_{z n_{0}}^{2}} \leq \frac{2}{\mu^{2} n_{z n_{0}}} \quad \forall x \in\right] n_{o}, n_{o}+1[.
$$

By combining all the bounds and (7d), we finally obtain, for each $x$ in $] n_{o}, n_{o}+1[$,

$$
\begin{aligned}
\mathcal{T}(x) & \leq\left(n_{z 0}+\frac{2}{\mu^{2} n_{z n_{0}}}\right), \\
& \leq\left(n_{z 0}+\frac{2 n_{0}^{2}}{\mu^{2} n_{z 1}}\right) \\
& \leq\left(n_{z 0}+\frac{2}{\mu^{2} n_{z 1}} x^{2}\right) .
\end{aligned}
$$

Case c) $n_{o}+1 \leq x$. Since the function $\chi \mapsto \frac{\chi+1}{(\chi-1)^{2}}$ is increasing on $[0,1[$ and decreasing on $] 1,+\infty[$, from (36), we obtain, for each $x$ larger than $n_{o}+1$,

$$
\begin{aligned}
\mathcal{T}(x) & =\frac{\sum_{\ell=0}^{n_{o}-1} \frac{\ell^{2}+x^{2}}{\left(x^{2}-\ell^{2}\right)^{2}} n_{z \ell}+\frac{n_{o}^{2}+x^{2}}{\left(x^{2}-n_{o}^{2}\right)^{2}} n_{z \ell}}{1+\mu^{2} x^{2}\left(\sum_{\ell=0}^{n_{o}} \frac{1}{x^{2}-\ell^{2}} n_{z \ell}\right)^{2}} \leq\left[\int_{0}^{n_{o}} \frac{x^{2}+\ell^{2}}{\left(x^{2}-\ell^{2}\right)^{2}} d \ell+\frac{\left(n_{o}+1\right)^{2}+n_{o}^{2}}{\left(\left(n_{o}+1\right)^{2}-n_{o}^{2}\right)^{2}}\right] n_{z 0} \\
& \leq n_{z 0}\left[\left.\frac{\ell}{x^{2}-\ell^{2}}\right|_{0} ^{n_{o}}+1\right] n_{z 0} \leq\left[\frac{n_{o}}{x^{2}-n_{o}^{2}}+1\right] n_{z 0} \leq\left[\frac{n_{o}}{\left(n_{o}+1\right)^{2}-n_{o}^{2}}+1\right] n_{z 0} \\
& \leq n_{z 0} \frac{3 n_{o}+1}{2 n_{o}+1} \leq \frac{3}{2} n_{z 0} .
\end{aligned}
$$


End of the proof. Finally, we can now conclude the proof of the lemma. For this, we combine bounds (140), (160), (161) and (162).

- For each $x$ integer in $\left\{0, \ldots, n_{o}\right\}$, inequality (140) gives

$$
\mathcal{T}(x) \leq \frac{2}{\mu^{2} n_{z 1}} x^{2}
$$

- For each $x$ in $(m, m+1)$ with $m$ in $\left\{0, \ldots, n_{o}-1\right\}$, inequality (160) gives

$$
\mathcal{T}(x) \leq\left(\frac{7}{2} n_{z 0}+\left[\frac{4 n_{z 0}}{\varpi^{2}}+\frac{512}{\mu^{2}} \frac{1}{n_{z 1}}\right] x^{2}\right),
$$

with $\varpi$ defined in (154) does not depend on $x$ nor $n_{o}$.

- For each $x$ in $] n_{o}, n_{o}+1[$, inequality (161)

$$
\mathscr{T}(x) \leq n_{z 0}+\frac{2}{\mu^{2} n_{z 1}} x^{2} .
$$

- For each $x$ larger than $n_{o}+1$, inequality (162) gives

$$
\mathfrak{T}(x) \leq \frac{3}{2} n_{z 0} .
$$

So we have established (139) with

$$
\kappa_{0}=\frac{7}{2} n_{z 0} \quad, \quad \kappa_{1}=\frac{4 n_{z 0}}{\varpi^{2}}+\frac{512}{\mu^{2}} \frac{1}{n_{z 1}}
$$

which do not depend on $n_{0}$, thus concluding the proof.

\section{References}

1. Afshar, S., Paunonen, L.: Adaptive robust output regulation control design. In: American Control Conference, pp. 3038-3043 (2019)

2. Astolfi, D., Marx, S., van de Wouw, N.: Repetitive control design based on forwarding for nonlinear minimum-phase systems. Automatica 129, 109671 (2021)

3. Astolfi, D., Praly, L.: Integral action in output feedback for multi-input multi-output nonlinear systems. IEEE Transactions on Automatic Control 62(4), 1559-1574 (2017)

4. Astolfi, D., Praly, L., Marconi, L.: Approximate regulation for nonlinear systems in presence of periodic disturbances. In: 54th IEEE Annual Conference on Decision and Control, pp. 7665-7670 (2015)

5. Astolfi, D., Praly, L., Marconi, L.: Francis-wonham nonlinear viewpoint in output regulation of minimum phase systems. IFAC-PapersOnLine 52(16), 532-537 (2019)

6. Bin, M., Astolfi, D., Marconi, L.: About robustness of control systems embedding an internal model. IEEE Transactions on Automatic Control, arXiv preprint arXiv:2010.08794 (2022)

7. Bin, M., Astolfi, D., Marconi, L., Praly, L.: About robustness of internal model-based control for linear and nonlinear systems. In: 57th IEEE Conference on Decision and Control, pp. 5397-5402 (2018)

8. Bin, M., Marconi, L., Teel, A.R.: Adaptive output regulation for linear systems via discrete-time identifiers. Automatica 105, 422-432 (2019)

9. Byrnes, C.I., Isidori, A.: Limit sets, zero dynamics, and internal models in the problem of nonlinear output regulation. IEEE Transactions on Automatic Control 48(10), 1712-1723 (2003)

10. Byrnes, C.I., Isidori, A.: Nonlinear internal models for output regulation. IEEE Transactions on Automatic Control 49(12), 2244-2247 (2004)

11. Byrnes, C.I., Priscoli, F.D., Isidori, A.: Output regulation of uncertain nonlinear systems. Springer Science \& Business Media (1997)

12. Cartan, H.: Differential calculus. Hermann (1983)

13. Davison, E.: The robust control of a servomechanism problem for linear time-invariant multivariable systems. IEEE transactions on Automatic Control 21(1), 25-34 (1976)

14. Deimling, K.: Ordinary differential equations in Banach spaces, vol. 596. Springer (2006) 
15. Del Vecchio, D., Marino, R., Tomei, P.: Adaptive learning control for feedback linearizable systems. European journal of control 9(5), 483-496 (2003)

16. Francis, B.A., Wonham, W.M.: The internal model principle of control theory. Automatica 12(5), 457-465 (1976)

17. Gao, W., Jiang, Z.P.: Learning-based adaptive optimal tracking control of strict-feedback nonlinear systems. IEEE transactions on neural networks and learning systems 29(6), 2614-2624 (2017)

18. Gentili, L., Marconi, L., Paden, B.: Disturbance rejection in the control of a maglev artificial heart. Journal of Dynamic Systems, Measurement, and Control 130(1), 011003 (2008)

19. Ghosh, J., Paden, B.: Nonlinear repetitive control. IEEE Transactions on Automatic Control 45(5), 949-954 (2000)

20. Hale, J.K.: Oscillations in nonlinear systems. Dover (1992)

21. Han, J.: From pid to active disturbance rejection control. IEEE transactions on Industrial Electronics 56(3), 900-906 (2009)

22. Hara, S., Yamamoto, Y., Omata, T., Nakano, M.: Repetitive control system: A new type servo system for periodic exogenous signals. IEEE Transactions on automatic control 33(7), 659-668 (1988)

23. Huang, J., Lin, C.F.: On a robust nonlinear servomechanism problem. IEEE Transactions on Automatic Control 39(7), 1510-1513 (1994)

24. Isidori, A.: Nonlinear control Systems. Springer-Verlag, New York (1995)

25. Kasac, J., Novakovic, B., Majetic, D., Brezak, D.: Passive finite-dimensional repetitive control of robot manipulators. IEEE Transactions on Control Systems Technology 16(3), 570-576 (2008)

26. Khalil, H.K.: On the design of robust servomechanisms for minimum phase nonlinear systems. International Journal of Robust and Nonlinear Control: IFAC-Affiliated Journal 10(5), 339-361 (2000)

27. Khalil, H.K.: Nonlinear systems. Prentice hall (2002)

28. Koçan, O., Astolfi, D., Poussot-Vassal, C., Mancey, A.: Supervised output regulation via iterative learning control for rejecting unknown periodic disturbances. In: 21st IFAC World Congress (2020)

29. Kurniawan, E., Cao, Z., Mahendra, O., Wardoyo, R.: A survey on robust repetitive control and applications. In: 2014 IEEE International Conference on Control System, Computing and Engineering (ICCSCE 2014), pp. 524-529 (2014)

30. Longman, R.W.: Iterative learning control and repetitive control for engineering practice. International journal of control 73(10), 930-954 (2000)

31. Mahmoud, N.A., Khalil, H.K.: Asymptotic regulation of minimum phase nonlinear systems using output feedback. IEEE Transactions on Automatic Control 41(10), 1402-1412 (1996)

32. Marconi, L., Praly, L.: Uniform practical nonlinear output regulation. IEEE Transactions on Automatic Control 53(5), 1184-1202 (2008)

33. Marconi, L., Praly, L., Isidori, A.: Output stabilization via nonlinear luenberger observers. SIAM Journal on Control and Optimization 45(6), 2277-2298 (2007)

34. Marino, R., Tomei, P.: An iterative learning control for a class of partially feedback linearizable systems. IEEE Transactions on Automatic Control 54(8), 1991-1996 (2009)

35. Marino, R., Tomei, P.: Online frequency estimation of periodic signals. In: Mathematical Control Theory I, pp. 257-276. Springer (2015)

36. Marino, R., Tomei, P.: Hybrid adaptive multi-sinusoidal disturbance cancellation. IEEE Transactions on Automatic Control 62(8), 4023-4030 (2016)

37. Martin, R.H.: Nonlinear operators and differential equations in Banach spaces. Krieger Publishing Co., Inc. (1986)

38. Mattavelli, P., Marafao, F.P.: Repetitive-based control for selective harmonic compensation in active power filters. IEEE Transactions on Industrial Electronics 51(5), 1018-1024 (2004)

39. Miller, R.K., Michel, A.N.: Ordinary differential equations. Academic Press (1982)

40. Nikiforov, V.O.: Adaptive non-linear tracking with complete compensation of unknown disturbances. European journal of control 4(2), 132-139 (1998)

41. Omata, T., Hara, S., Nakano, M.: Nonlinear repetitive control with application to trajectory control of manipulators. Journal of Robotic systems 4(5), 631-652 (1987)

42. Paunonen, L.: Robust controllers for regular linear systems with infinite-dimensional exosystems. SIAM Journal on Control and Optimization 55(3), 1567-1597 (2017)

43. Paunonen, L., Pohjolainen, S.: Internal model theory for distributed parameter systems. SIAM Journal on Control and Optimization 48(7), 4753-4775 (2010)

44. Serrani, A., Isidori, A., Marconi, L.: Semi-global nonlinear output regulation with adaptive internal model. IEEE Transactions on Automatic Control 46(8), 1178-1194 (2001)

45. Sontag, E.D.: Input to state stability: Basic concepts and results. In: Nonlinear and optimal control theory, pp. 163-220. Springer (2008) 
46. Teel, A., Praly, L.: Tools for semiglobal stabilization by partial state and output feedback. SIAM Journal on Control and Optimization 33(5), 1443-1488 (1995)

47. Tucsnak, M., Weiss, G.: Observation and control for operator semigroups. Springer (2009)

48. Verrelli, C.M.: A larger family of nonlinear systems for the repetitive learning control. Automatica 71, 38-43 (2016)

49. Wang, Y., Gao, F., Doyle III, F.J.: Survey on iterative learning control, repetitive control, and run-torun control. Journal of Process Control 19(10), 1589-1600 (2009)

50. Xu, D., Huang, J., Jiang, Z.P.: Global adaptive output regulation for a class of nonlinear systems with iiss inverse dynamics using output feedback. Automatica 49(7), 2184-2191 (2013) 